\title{
A ROBUST 4D TREATMENT PLANNING APPROACH FOR LUNG RADIOTHERAPY
}

\author{
by \\ Chandra P. Pokhrel \\ A thesis \\ presented to Ryerson University \\ in partial fulfillment of the \\ requirements for the degree of \\ Master of Science \\ in the Program of \\ Biomedical Physics
}

Toronto, Ontario, Canada, 2013

(C) Chandra P. Pokhrel 2013 


\section{Author's Declaration}

I hereby declare that I am the sole author of this thesis. This is a true copy of the thesis, including any required final versions, as accepted by my examiners.

I authorize Ryerson University to lend this thesis to other institutions or individuals for the purpose of scholarly research.

I further authorize Ryerson University to reproduce this thesis by photocopying or by other means, in total or in part, at the request of other institutions or individuals for the purpose of scholarly research.

I understand that my thesis may be made electronically available to the public.

Chandra P Pokhrel

September, 2013 


\begin{abstract}
A Robust 4D Treatment Planning Approach for Lung Radiotherapy

Chandra P. Pokhrel

Master of Science, Medical Physics

Ryerson University, 2013
\end{abstract}

4D treatment plans are optimized to compensates for a patient's individual respiratory motion. However, changes in the time spent in the each respiratory state may render a $4 \mathrm{D}$ plan invalid. We introduced and evaluate two robust treatment planning approaches to compensate for respiratory motion variations. Robust 4D plans can be designed either using an average pdf approach or a worst case pdf approach. We tested these approaches on two motion variation scenarios and compared them with nominal 4D optimization and ITV plans. The nominal 4D plans were very sensitive to the variation in respiration pattern while the average pdf and worst case pdf plans were less sensitive under the similar variations. The average and worst case pdf plans were not as robust as the ITV plans but had better healthy tissue sparing. The worst case pdf approach was found to be the most promising for robust $4 \mathrm{D}$ plan optimization. 


\section{Acknowledgements}

I would like to sincerely thank my supervisor Dr. Emily Heath for her constant support, time, and guidance in completing this project.

I would also like to thank committee members Dr. Pedro Goldman of Ryerson University and Dr. Harald Keller of Princess Margaret Hospital for their time and valuable comments.

Lastly, I would like to acknowledge the continual support of my family.

Funding for this project was provided by the NSERC Collaborative Health Research Program. (PI: T. Chan, University of Toronto) 
my mom whom I love so very much. 


\section{Table of Contents}

1 Introduction 1

1.1 Background . . . . . . . . . . . . . . . . 1

1.2 Radiation therapy . . . . . . . . . . . . . . . . . 3

1.2.1 Brachytherapy ................... 3

1.2.2 External beam radiotherapy . . . . . . . . . . . . . 4

1.3 External beam radiation therapy processes . . . . . . . . . . . . 4

1.4 Treatment target volume definitions . . . . . . . . . . . . . . 7

1.5 Dose deposition . . . . . . . . . . . . . . . 8

1.6 Intensity modulated radiation therapy $($ IMRT) $\ldots \ldots \ldots$. . . . . . . 13

1.7 Characteristics of respiratory motion . . . . . . . . . . 17

1.8 Effects of motion . . . . . . . . . . . . . . . 19

1.8.1 Effect on imaging . . . . . . . . . . . . . . . 19

1.8 .2 Dose blurring . . . . . . . . . . . . . . . . 20

1.8 .3 Interplay effect . . . . . . . . . . . . . . . . . 21

1.8.4 Dose deformation effect . . . . . . . . . . . . 22

1.9 Respiratory motion information: $4 \mathrm{D} \mathrm{CT} \ldots \ldots \ldots . \ldots . \ldots 23$

1.10 Respiratory motion management techniques . . . . . . . . . . . . . 24

1.10 .1 Motion encompassing method . . . . . . . . . . . . . 24

1.10 .2 Breath hold . . . . . . . . . . . . . . . . . . 24 
1.10 .3 Respiratory gating . . . . . . . . . . . . . . . 26

1.10.4 Real time tumor tracking . . . . . . . . . . . . 26

$1.10 .54 \mathrm{D}$ optimization $\ldots \ldots \ldots \ldots . \ldots \ldots$

1.11 Motion variations . . . . . . . . . . . . . . . . . . 29

1.12 Robust optimization . . . . . . . . . . . . . . . . 32

1.13 Hypothesis and objective . . . . . . . . . . . . . 34

2 Materials and methods $\quad 36$

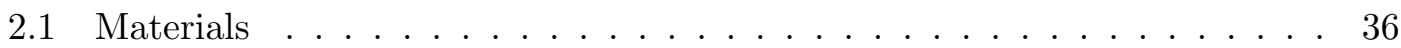

2.1 .1 XCAT phantom $\ldots \ldots \ldots \ldots \ldots$

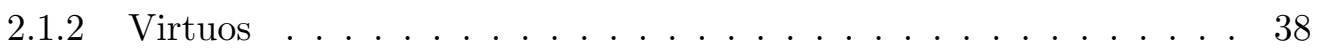

$2.1 .3 \mathrm{KonRad} \ldots \ldots \ldots \ldots . \ldots \ldots$

2.2 Treatment planning methods . . . . . . . . . . . . . . . 46

2.2 .1 ITV $\operatorname{Tan} \ldots \ldots \ldots \ldots \ldots \ldots$

2.2.2 Nominal 4D plan optimization . . . . . . . . . . . . . 46

2.2.3 Robust 4D plan optimization . . . . . . . . . . . . . . 49

2.3 Validation of $4 \mathrm{D}$ optimization . . . . . . . . . . . . . . 51

2.4 Motion variation scenarios . . . . . . . . . . . . . . . . 52

2.5 Testing sensitivity of plans to motion variations . . . . . . . . . . 55

2.6 Dose prescription and OAR dose tolerance . . . . . . . . . . . . 56

$\begin{array}{lll}3 & \text { Results and discussion } & 57\end{array}$

3.1 Validation test results . . . . . . . . . . . . . . 57

3.1 .1 ITV plan applied to 4DDIJ . . . . . . . . . . . 57

3.1 .2 CTV plan applied to 4DDIJ . . . . . . . . . . . 57

3.1 .3 Tracking plan . . . . . . . . . . . . . . . . . . 59

3.2 Motion variation scenario 1: more time inhaling vs exhaling . . . . . . 60

3.2 .1 Nominal $4 \mathrm{D}$ plan . . . . . . . . . . . . . . . . . . . 61 
3.2.2 Robust plan using average pdf . . . . . . . . . . . . . . 63

3.2.3 Robust plan using worst case pdfs . . . . . . . . . . . . . . 65

3.2 .4 ITV plan: . . . . . . . . . . . . . . . . . . 66

3.3 Motion variation scenario 2 : Amplitude variation . . . . . . . . . . 70

3.3 .1 Nominal $4 \mathrm{D}$ plan . . . . . . . . . . . . . . . . . . . 70

3.3.2 Robust plan using average pdf . . . . . . . . . . . . . 71

3.3.3 Robust plan using worst case pdf . . . . . . . . . . . . . . . . 74

3.3 .4 ITV plan . . . . . . . . . . . . . . . . 77

4 Conclusions and future work $\quad 80$

4.1 Summary and conclusions . . . . . . . . . . . . . . . 80

4.2 Future work . . . . . . . . . . . . . . . . . . 81

$\begin{array}{ll}\text { Bibliography } & 83\end{array}$ 


\section{List of Tables}

2.1 Tumor displacement . . . . . . . . . . . . . . . . . . . . . 52

2.2 Tumor motion variation scenario: more time inhaling vs exhaling . . . . . 53

2.3 Tumor motion variation scenario: amplitude variation . . . . . . . . . 54

3.1 Dose statistics for Nominal 4D plan applied to the motion variations in scenario 1. . . . . . . . . . . . . . . . . . . 62

3.2 Dose statistics for average-pdf plan applied to cases in motion variation scenario $1 \ldots \ldots \ldots \ldots$. . . . . . . . . . . . . . . 63

3.3 Dose statistics for worst case pdf plan applied to motion variation scenario

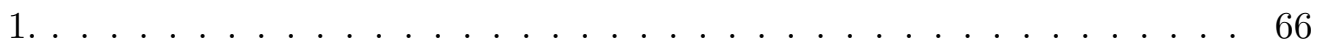

3.4 Dose statistics for ITV plan applied to motion variations scenario 1. . . . 68

3.5 Beam intensity statistics for (a) nominal (b) average pdf (c) worst case pdf and (d) ITV plan. . . . . . . . . . . . . . . . . . . 70

3.6 Dose statistics for nominal 4D plan applied to amplitude variation scenario. 71

3.7 Dose statistics of average pdf plan applied to amplitude variation scenario. 72

3.8 Dose statistics for worst case pdf plan (combination ratio 70/30) applied to amplitude variation scenarios. . . . . . . . . . . . 76

3.9 Dose statistics for worst case pdf plan (combination ratio 80/20) applied to amplitude variation scenarios. . . . . . . . . . . . . 76

3.10 Dose statistics for ITV plan applied to amplitude variation scenario. . . . 79 


\section{List of Figures}

1.1 Percentage distribution of cancer incidence and deaths in male (a) and

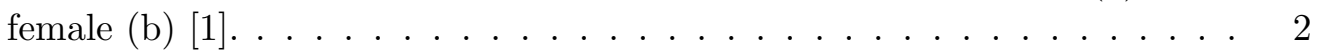

1.2 (a) Picture of a medical linear accelerator and (b) Schematics of the linac

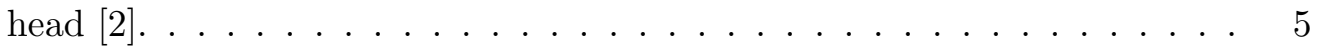

1.3 Multi leaf collimator used to shape the radiation beam [3] . . . . . . . . 5

1.4 Graphical representation of treatment target volumes [4]. . . . . . . . . . 8

1.5 Schematics of dose change as a function of depth [2]. . . . . . . . . . . . . 10

1.6 Central axis dose distribution expressed in terms of percentage-depth-dose

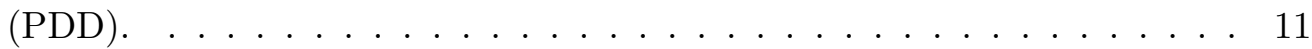

1.7 $10 \mathrm{MV}$ x-ray beam profile at various depth of water for field size of $10 \times 10$ and $30 \times 30 \mathrm{~cm}^{2}$ and source to surface distance (SSD) $=100 \mathrm{~cm} \mathrm{[4]} \mathrm{.} \mathrm{.} \mathrm{.} \mathrm{.} 12$

1.8 Illustration of beam intensity profile in 3DCRT (a) and in IMRT (b) [5]. . 15

1.9 Effect of tumor motion on imaging . . . . . . . . . . . . . 20

1.10 Illustration of dose blurring due to organ motion . . . . . . . . . . . . 21

1.11 Illustration of interplay effect due to motion of organ relative to leafs of MLC [6]. . . . . . . . . . . . . . . . . . . . . . . . . . . 22

1.12 The mechanism of acquiring 4D CT images. . . . . . . . . . . . . 23

1.13 Graph shows that deep inspiration breath-hold increases the lung volume for extended period of time [7]. . . . . . . . . . . . . . . 25

1.14 The Breathing pattern variation of a patient. . . . . . . . . . . . 30

1.15 The motion pdf variation of a representative patient in different treatment fractions. . . . . . . . . . . . . . . . . . 30

1.16 Effect of motion uncertainty in the treatment . . . . . . . . . . . . 31

1.17 Model of motion uncertainty as proposed by Chan et al. . . . . . . . . . 33

1.18 Robust optimization using model of uncertainty in motion pdf . . . . . . 34

2.1 Anterior view of male (left) and female (right) anatomies of 4D XCAT phantom $[8] \ldots \ldots \ldots$. . . . . . . . . . . . . . . . . . . . . 37

2.2 Reconstructed transverse CT slice simulated using XCAT phantom with a spherical lesion of diameter of $30 \mathrm{~mm}$ in the right lung. . . . . . . . . . 39

2.3 Virtuos image processing mode showing the delineation of different volumes of interest in transverse, sagital, beam's eye view and observer's view. Legend: Green-GTV, red-CTV, yellow-ITV, magenta-lungs, orangeheart, pink-spinal cord, blue- body. . . . . . . . . . . . . . . . . . 40 
2.4 Virtuos in planning mode showing the 3D patient model in observer's view, and $2 \mathrm{D}$ transverse view of beams arrangements with different parameters such as beam energy and gantry angles. . . . . . . . . . . . . . . . 41

2.5 Virtuos in result mode allows to evaluate the the treatment plan by providing DVHs, dose statistics, and dose distributions. . . . . . . . . . . 42

2.6 Illustration of relationship between bixels and voxels [9]. . . . . . . . . . 43

2.7 Graphical user interface of KonRad treatment plan optimization system allows to choose maximum and minimum dose limits for different organs.

2.8 A contour (magenta) encompassing GTV of inhale and exhale phases is expanded by $5 \mathrm{~mm}$ to get an ITV (red). . . . . . . . . . . . . . 47

2.9 Illustration of anatomical voxel movement when tumor moves. Arrow indicates deformation vector for voxel $(i, j, k)$. Motion information given by the vector is used to match the corresponding voxels. . . . . . . . . . . 49

2.10 Bixel intensity maps in 'worst-case' pdf approach . . . . . . . . . . . . . . 51

2.11 Tumor displacement in a respiration cycle of $5 \mathrm{sec}$ as generated by XCAT. 52

2.12 Illustration of phases in a respiration cycle. The numbers indicate the different phases of tumor motion, and the ellipsoid represents the periodic nature of the motion but not the trajectory. . . . . . . . . . . . 54

3.1 ITV plan applied to Exhale phase. Dashed yellow line indicate 95\%(57 Gy) isodose line. Pinl dots represent the ITV. . . . . . . . . . . . 58

3.2 Accumulated dose over 10 phases from ITV plan. Dashed yellow line indicate 95\%(57 Gy) isodose line. Red dots represent the exhale CTV. . . 58

3.3 Dose distribution of CTV plan on Exhale phase. Dashed yellow line indicate 95\%(57 Gy) isodose line. Red dots represent exhale CTV. . . . . . . 58

3.4 Accumulated dose distribution for CTV plan. Dashed yellow line indicate 95\%(57 Gy) isodose line. Red dots represent exhale CTV. . . . . . . . . 58

3.5 Dose distribution of tracking plan on Inhale phase. Red dots represent the CTV in inhale. . . . . . . . . . . . . . . . . . 59

3.6 Accumulated dose distribution of tracking plan. Red dots represent the CTV in exhale. . . . . . . . . . . . . . . . . . . 59

3.7 Figures showing the imperfect image registration. (a) Inhale image and (b) inhale image subtracted from transformed exhale to inhale phase. Yellow and red color represent a positive image difference. . . . . . . . . . . . . . 60

3.8 (a) The tumor displacement as a function of time and (b) phase weight as a function of phase. . . . . . . . . . . . . . . 61

3.9 Dose volume histograms (DVH) showing the sensitivity of a nominal $4 \mathrm{D}$ plan to motion variation scenario 1 . The vertical dashed lines represent ICRU min and $\max$ dose criteria. . . . . . . . . . . . . . . . . 62

3.10 Dose volume histograms showing the sensitivity of average pdf plan under motion variation scenario 1 . Vertical dashed lines indicate ICRU $\min / \max$ dose criteria. . . . . . . . . . . . . . . . . . . 64 
3.11 Dose distribution when average pdf plan is applied to case14. Yellow dashed line indicates $95 \%$ of prescribed dose. Red dots represent CTV at exhale. . . . . . . . . . . . . . . . . . . . . 64

3.12 Dose volume histogram of robust plan using worst case approach in motion variation scenario 1 . Vertical dashed lines represent ICRU $\min / \max$ dose criteria. . . . . . . . . . . . . . . . . 66 66

3.13 Dose volume histograms of ITV plan under motion variation scenario 1 . Vertical dashed lines represent ICRU $\min / \max$ dose criteria. . . . . . . . 66

3.14 Dose distribution when ITV plan is applied to case17. Pink dots represent exhale CTV. . . . . . . . . . . . . . . . . . . 67

3.15 Dose distribution when average pdf plan is applied to case17. Pink dots represent exhale CTV. . . . . . . . . . . . . . . . 67 67

3.16 Left to right: beam intensity profiles of plans in nominal, average pdf, worst case pdf and ITV approach. Top to bottom: beam 1 to beam 5. . . 69

3.17 Tumor motion amplitude variation scenarios. The bold lines represent worst case pdfs. . . . . . . . . . . . . . . . 71

3.18 CTV DVH of nominal plan under amplitude variation. . . . . . . . . 73

3.19 CTV DVH of average pdf plan under amplitude variation. . . . . . . . 73

3.20 Sagital view of dose distribution when average pdf plan is applied to case24. 73

3.21 Sagital view of dose distribution when average pdf plan is applied to case23. 73

3.22 Dose distribution of (a) nominal and (b) average pdf plan applied to exhale CTV. Pink dots represent exhale CTV. . . . . . . . . . . . . . 74

3.23 Dose volume histogram of wc pdf plan (50/50 ratio) under amplitude variation. . . . . . . . . . . . . . . . . . 75

3.24 Dose volume histogram of wc pdf plan (70/30 ratio) under amplitude variation. . . . . . . . . . . . . . . . . 75

3.25 Dose volume histogram of wc pdf plan (80/20 ratio) under amplitude variation. . . . . . . . . . . . . . . . . . 75

3.26 Dose volume histogram of wc pdf plan (60/40 ratio) under amplitude variation. . . . . . . . . . . . . . . . . . 75

3.27 Dose distribution when worst case pdf plan (80-20 combination) is applied to case23. Yellow dashed line indicates $95 \%$ isodose. Pink dots represent the exhale CTV. . . . . . . . . . . . . . . . . 77

3.28 Dose volume histograms of the ITV plan under amplitude variation. . . . 78

3.29 Dose distribution of the ITV plan on exhale phase. . . . . . . . . . . 78

3.30 Accumulated dose distribution of the ITV plan applied to case24. . . . . 78 


\section{Chapter 1}

\section{Introduction}

\section{$1.1 \quad$ Background}

Each cell in our body contains DNA that carry genes. Sequences of genes inside DNA govern the cell function. Normal cells grow, divide, and die when they get old in a controlled fashion as instructed by the gene. When DNA gets damaged either by radiation or by chemical reaction, it may result in a mutation. In an ideal case, when the cell replicates the resulting sister cell is identical to the parent cell. But when mutation occurs, the resulting sister cell is different from the parent cell because faulty DNA alters the gene structure. The faulty gene is called an oncogene. When the oncogene prevents the cell from undergoing apoptosis, programmed cell death, the cell does not die but grows out of control to produce a tumor. Tumors can be malignant (cancerous) or benign (non-cancerous). Benign tumor cells do not invade adjacent tissue. However, malignant tumor cells are invasive. If cancer spreads to other part of the body it is called metastatic cancer. Substances that are responsible for damaging DNA and promoting cancer are called carcinogens. Tobacco, asbestos, arsenic, and radiation are examples of carcinogens. Faulty genes inherited from the parents can also result in cancer.

Cancer is named according to the body part where it grows. The most common 
cancer in men is prostate cancer and in women is breast cancer. Lung cancer is the second most common cancer both in men and women but it ranks first in terms of mortality both in men and women attributing, respectively, to $27 \%$ and $26 \%$ of the total deaths due to cancer (Figure 1.1 [1]). Lung cancer starts and grows in the lungs and may spread to other parts of the body. There are two types of lung cancers based on the appearance of the cancer cells when seen under a microscope: non-small cell lung cancer (NSCLC) and small cell lung cancer (SCLC). $85 \%$ to $90 \%$ of all lung the cancers are non-small cell lung cancer and $10 \%$ to $15 \%$ are small cell lung cancer [1]. Lung cancer is classified into different stages depending on how far it has spread. The stage is defined by tumor-node-metastasis (TNM) $[10,11]$ classification system where

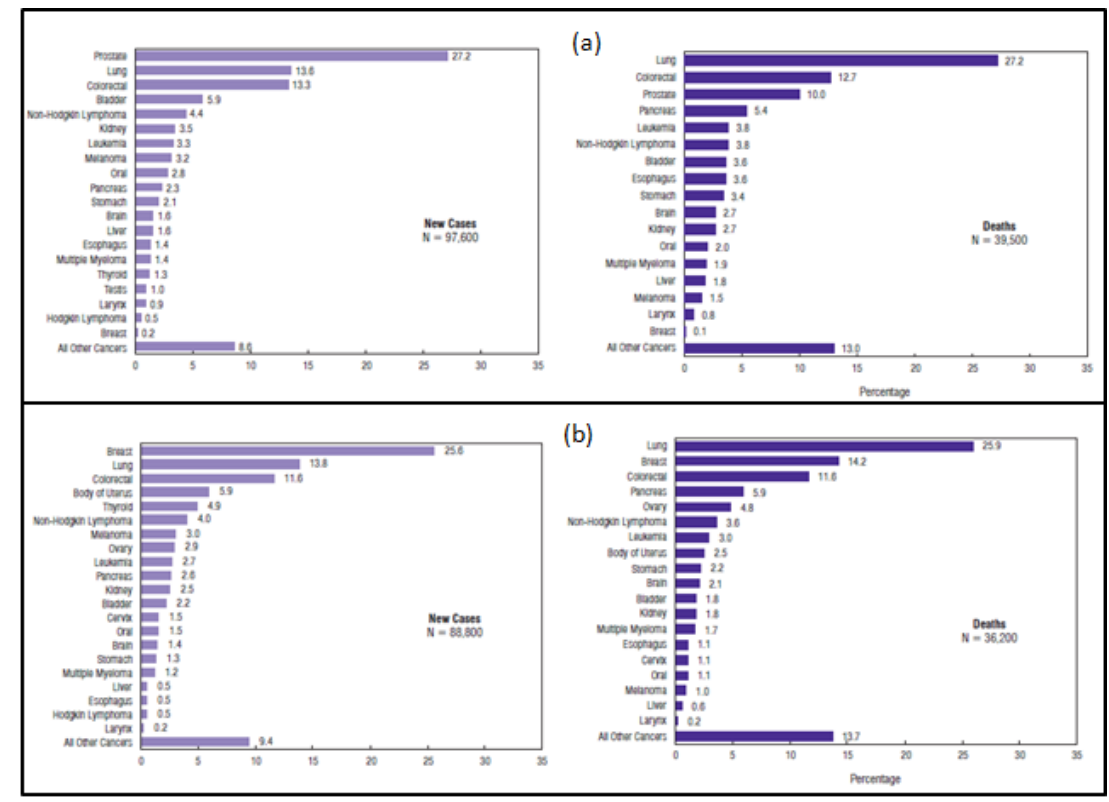

Figure 1.1: Percentage distribution of cancer incidence and deaths in male (a) and female (b) $[1]$.

- $\mathrm{T}$ indicates the size of the main(primary) tumor and whether it has grown to nearby areas.

- $\mathrm{N}$ indicates the spread of cancer to nearby lymph nodes. 
- M indicates when cancer has metastasized and spread to other parts of the body.

The most common sites for metastases are the brain, liver, kidney, other lung and adrenal gland. The letters T,N,M indicate each of the above conditions and number 0 through 4 indicate increasing severity.

There are different treatment methods for lung cancer. These methods include surgery, chemotherapy, radiation therapy, laser surgery, photodynamic therapy, and cryotherapy $[12,13]$. Choosing a method for a particular patient depends on the stage of cancer and overall health condition.

\section{$1.2 \quad$ Radiation therapy}

Radiation therapy is the use of ionizing radiation to kill cancer cells by damaging their DNA. Radiation can damage DNA directly or it may damage indirectly by producing free radicals inside the cell. High energy radiation can be either x-rays, gamma rays or charged particles such as protons. There are two ways the radiation can delivered to the patient: from outside the body (external beam radiotherapy) or by placing a source of radiation inside or close to the tumor inside the body (brachytherapy).

\subsubsection{Brachytherapy}

Brachytherapy is an internal radiation treatment method in which sources of radioactive material are implanted in or near the tumor. Radioactive material is sealed in a small metal capsule and implanted with the help of needle or wire. The implanted source is either removed after a short time or remains permanently in the body. This method makes it possible to give a highly localized radiation dose with sharp dose fall off in the surrounding normal tissue. The radioactive sources used for lung cancer brachytherapy are Iodine $\left({ }^{125} \mathrm{I}\right)$, Ytterbium $\left({ }^{169} \mathrm{Yb}\right)[14]$, Palladium $\left({ }^{103} \mathrm{Pd}\right)$, Cesium $\left({ }^{137} \mathrm{Cs}\right)$, Iridium $\left({ }^{192} \mathrm{Ir}\right)$, Gold $\left({ }^{198} \mathrm{Au}\right)$, Cobalt $\left({ }^{60} \mathrm{C}\right)$, and Radium $\left({ }^{226} \mathrm{Ra}\right)[15]$. 


\subsubsection{External beam radiotherapy}

In external beam radiotherapy of lung cancer, the tumor is irradiated by high energy radiation from a source outside the patient's body. The radiation source is either a medical linear accelerator (linac) for x-rays, Cobalt-60 unit for gamma rays or a synchrotron or a cyclotron to generate a proton beam.

In a linac (Figure 1.2) electrons emitted from the electron gun are accelerated to high energies by high frequency electromagnetic fields within a waveguide. The accelerated electrons strike a target of high atomic number material such as tungsten. Some of the incident electrons interact with the nucleus of the target atom giving off Bremsstrahlung $\mathrm{x}$-rays, and a few of them eject electrons from the inner orbits of the target atom giving off characteristic x-rays. X-rays coming out of the target have a spectrum of energies ranging up to a few $\mathrm{MeV}$. The X-ray beam coming out of the target is first collimated by a primary collimator and then passes through a flattening filter. The flattening filter makes the beam intensity uniform. The flattened beam then passes through the dose monitoring chamber (ion chamber). After the dose monitoring unit the x-ray beam is further collimated by adjustable secondary collimators before hitting the patient. Modern accelerators include tertiary collimators known as multi-leaf-collimators (MLC). The collimator is made up of large number of individual leafs of high atomic number material such as tungsten. The leaves of the collimator are capable of moving independently in and out of the path of the radiation beam in order to block and shape according to the shape of the target volume. Figure 1.3 shows a multi-leaf collimator by Varian Medical Systems Inc (Palo Alto, CA).

\subsection{External beam radiation therapy processes}

After the diagnosis of cancer and the decision to use radiation therapy is made the external radiation therapy treatment process involves the steps discussed below. 

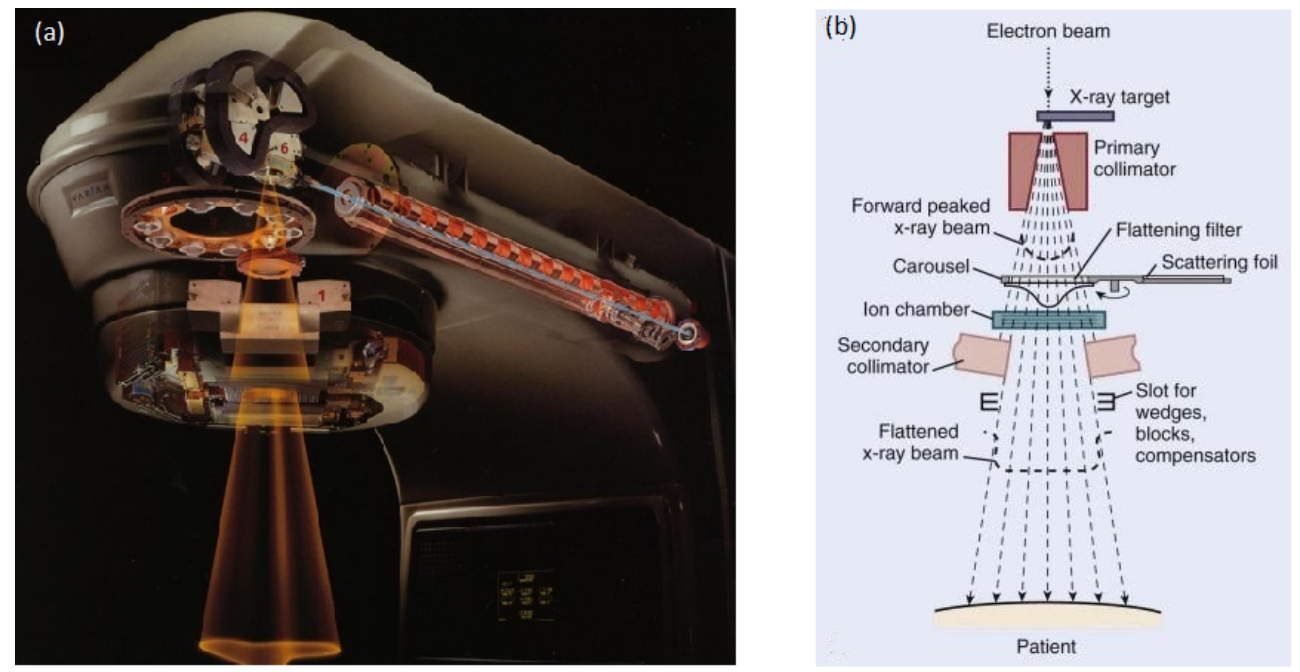

Figure 1.2: (a) Picture of a medical linear accelerator and (b) Schematics of the linac head [2].

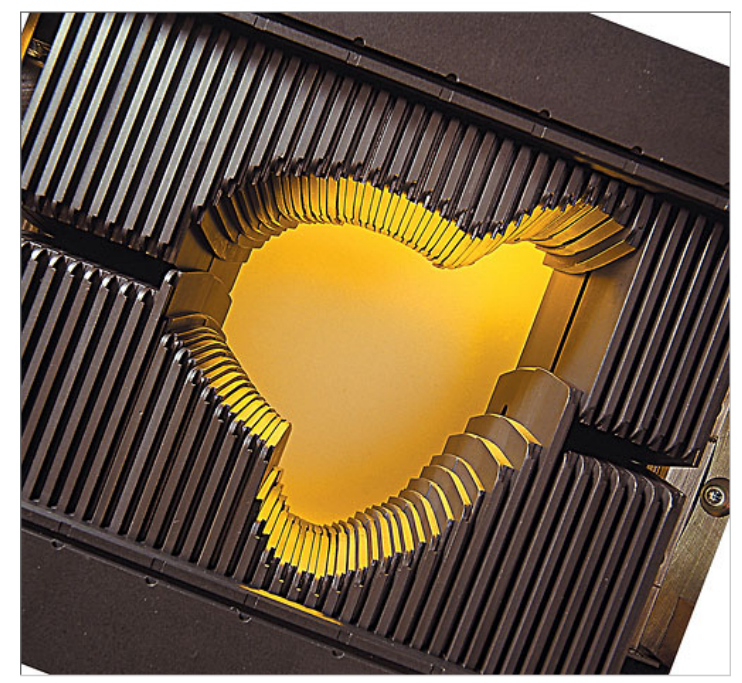

Figure 1.3: Multi leaf collimator used to shape the radiation beam [3]. 
1) Target localization: The term 'target localization' means the determination of the exact anatomical position and extent of the treatment volume, and referencing of that volume with respect to surface markers in order to guide alignment of the patient on the treatment machine. CT and MR images are acquired of the patient in the treatment position, and the tumor and organs at risk are contoured by the physician.

2) Treatment simulation: A treatment simulation is a trial set up of patient in a simulator that mimics the geometry of the treatment machine. Selection of the number of beams, their direction and size are simulated using a light field and the radiographic or fluoroscopic mode to mimic the real radiotherapy beams in terms of divergence and tissue exposed. The final beam configuration is recorded permanently by taking a radiograph. This trial setup is not recommended on a treatment machine because, firstly, the quality of images from high energy x-ray beams is poor, and, secondly, the process is time consuming and therefore engages the therapy machine for a prolonged period of time. In order to make sure that beam placement is not changing during the treatment, immobilization devices are also made during simulation. To indicate the exact spot where radiation should be aimed, markers are drawn or placed on the patient skin using marker. These marks are either temporary which can be washed off after the treatment is completed or permanent small tattoos. Modern treatment simulation is performed virtually in software known as a 'virtual simulator' using planning CT images.

3) Treatment planning: Patient specific data obtained from the CT scan is transfered to a planning computer. Then $3-\mathrm{D}$ treatment planning software is used to design the number of beams and their geometrical arrangements, and calculate the dose distribution. Typically treatment planning is an iterative process. Adjustments are made until the dose objectives for the target volume and organs at risk are met. The ICRU [16] states 
that the target volume dose should not be less than $95 \%$ or greater than $107 \%$ of the prescribed dose.

4) Treatment plan verification: Before a plan is delivered it is checked for its physical and dosimetric integrity to make sure that (a) the plan is deliverable and (b) there are no errors in the planning process. Verifications include a manual check of dose calculations and delivery of the plan to a phantom to check the dose delivery.

5) The final step in radiotherapy is the treatment delivery on the medical linear accelerator. Photon beam radiation treatment normally takes 15 to 20 minutes for each fraction including time to set up patient, patient position verification, and delivery of multiple beams.

\subsection{Treatment target volume definitions}

In radiation therapy it is important to precisely define the treatment volumes so that the tumor is fully irradiated while sparing healthy tissue as much as possible. The actual treated volume is larger than what is seen on an x-ray or CT image or observed by other

diagnostic tools. Guidelines for target volume definitions are given in ICRU report 50 and $62[16,17]$.

1. The gross tumor volume (GTV) is

"the gross palpable or visible/demonstrable extent and location of malignant growth" (ICRU Report No. 50). The GTV is usually based on the information from a different imaging modalities such as CT, MRI, and ultrasound. [4].

2. Clinical target volume (CTV)

"A margin is added around the Gross Tumor Volume (GTV) to take into account potential sub-clinical invasion. The GTV and this margin defines the Clinical Tar- 


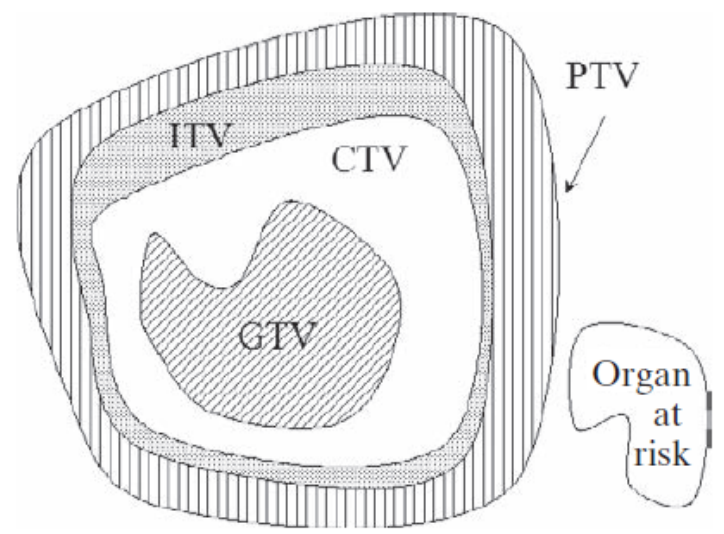

Figure 1.4: Graphical representation of treatment target volumes [4].

get Volume (CTV)".

3. Internal target volume (ITV)

The ITV is defined as the volume that encompasses the CTV plus an internal margin (IM). The internal margin is added to account variation in shape, size and position of the CTV due to organ motion such as breathing. Thus, ITV=CTV+ IM (ICRU report 62).

4. Planning target volume (PTV)

A set-up margin (SM) is further added to the ITV in order to account for any variations in the patient setup between fractions. Thus, PTV=ITV+SM. (ICRU report 62$)$.

\subsection{Dose deposition}

The mechanism of energy deposition by ionizing radiation depends on the type of radiation. Indirectly ionizing radiation such as x-rays and gamma rays deposit energy in the medium in a two step process. In the first step, photons transfer their energy to charged particles in the medium. In the second step the charged particles transfer their energy 
to the medium through Coulomb interactions. Charged particles (protons, electrons) deposit their energy in matter directly through Coulomb interactions along its track in the medium $[4,18]$. Energy deposited into the medium by ionizing radiation radiation is measured in terms of absorbed dose. Dose is defined as the mean energy imparted by the charged particle to the medium per unit mass $m$ in a finite volume $V$ [18].

$$
D=d E / d m
$$

The unit of dose is $\mathrm{J} / \mathrm{kg}$ or Gy. The total kinetic energy transfered to the charged particles in the matter before any energy is deposited by charged particles is measured in terms of Kerma.

$$
K=d E_{t r} / d m
$$

The unit of kerma is Joule/kg. The kinetic energy of the charged particles in the medium (i.e kerma) may be expended in two ways. It may be expended by collisions with atomic electrons resulting in ionization and excitation of the medium along its path or by Coulomb interactions with the atomic nuclei. Hence, kerma can be divided into two parts- collisional kerma denoted by $K_{c}$ and radiative kerma denoted by $K_{r}$.

$$
K=K_{c}+K_{r}
$$

If the energy is lost through Coulomb interactions with the nucleus, charged particle slows down giving off bremsstrahlung radiation which mostly escapes from the medium. For mono-energetic photons the collisional kerma at a point in the medium is related to the energy fluence $\Psi$ by the equation

$$
K_{c}=\Psi\left(\frac{\mu_{a b}}{\rho}\right)=K(1-g)
$$




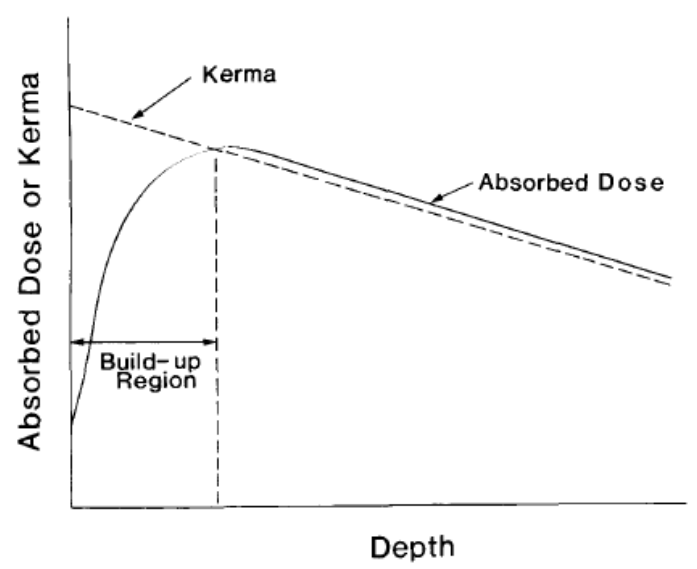

Figure 1.5: Schematics of dose change as a function of depth [2].

where the bremsstrahlung factor $g$ defines the fraction of the radiative loss of energy, and $\mu_{a b} / \rho$ is the mass-energy absorption coefficient for mono-energetic photons in the medium. The energy fluence $\Psi$,

$$
\Psi=h \nu . \Phi
$$

depends on the particle fluence

$$
\Phi=d N / d A
$$

Similarly, the total kerma $K$ at a point in the medium is related to the energy fluence $\Psi$ by the equation

$$
K=\Psi\left(\frac{\mu_{t r}}{\rho}\right)
$$

where $\mu_{t r} / \rho$ is mass-energy transfer coefficient. Since radiative photons mostly escape from the medium, the dose deposited in the medium is mainly due to collisional kerma. In general, the relation between collisional kerma and dose is

$$
\beta=D / K_{c}
$$

Generally, transfer of energy from photons to charged particles does not lead to 


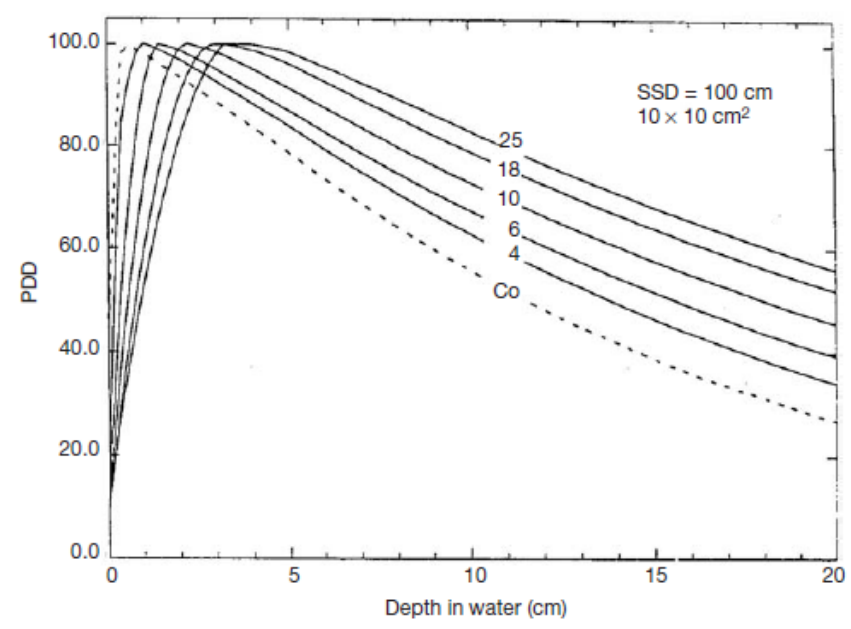

Figure 1.6: Percentage-depth-dose (PDD) distribution in water for photon beams with energies ranging from ${ }^{60} \mathrm{Co} \gamma$ rays to $25 \mathrm{MV}$ x-rays for a $10 \mathrm{x} 10 \mathrm{~cm}^{2}$ field at a source to surface distance (SSD) of $100 \mathrm{~cm}$ [4]. The labels on the curves indicate the beam energy in MV.

absorption of energy at the same point because charged particles have finite range. As high energy photons are incident on the surface of the medium, they produce charged particles at the surface and at the subsequent layers in their way. At the entrance surface of the medium the photon fluence is maximum; then it slowly decreases with depth. However, charged particle fluence and hence the dose slowly increases with depth until they reach maximum at depth of $d_{\max }$ because electrons deposit their energy at a significant distance away from the site of origin. After $d_{\max }$, the electron fluence decreases and hence the dose. Figure 1.5 [2] shows the variation of dose in medium with depth. The region from the surface to the point of maximum dose is called the build up region. The build up region is responsible for the skin-sparing effect. The central axis dose distribution inside the patient is usually normalized to maximum dose $D_{\max }=100 \%$ at the depth of $d_{\max }$ and is referred to as percentage-depth-dose (PDD) curve. The PDD is different for photon beams of different energy as shown in Figure 1.6 [4]. As the photon energy increases the build up region increases. This shows a clear advantage of using higher energy beam over lower energy beam for which maximum dose 


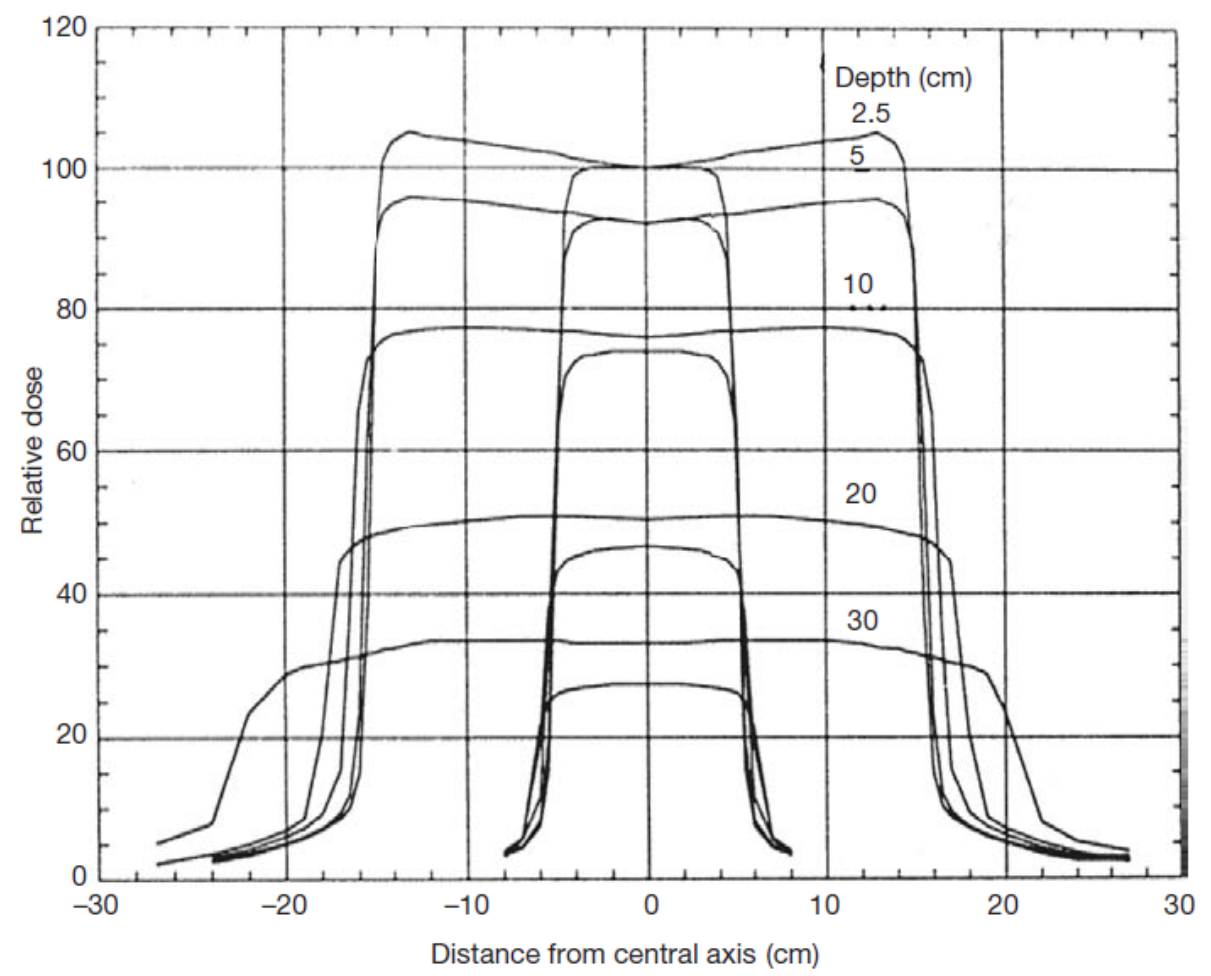

Figure 1.7: $10 \mathrm{MV}$ x-ray beam profile at various depth of water for field size of 10x10 and $30 \times 30 \mathrm{~cm}^{2}$ and source to surface distance $(\mathrm{SSD})=100 \mathrm{~cm} \mathrm{[4]}$.

$D_{\max }$ occurs closer to the surface. That is why deep-seated tumors require high energy beam so that maximum dose could be delivered without exceeding the skin tolerance.

Dose profiles along the beam axis combined with off axis dose profile gives the complete picture of dose distribution in the target volume. Plotting the variation across the field at a specific depth produces an off-axis beam profile. Typical beam profiles at various depths of water for MV x-ray beam of 10x10 and 30x30 $\mathrm{cm}^{2}$ field size are shown in Figure 1.7 [4]. The dosimetric field size is defined as the lateral distance between the $50 \%$ dose level points on the beam profile. There are three regions of the beam profile: central, penumbra and umbra. The central region represents the central portion of the beam profile extending from beam central axis to within 1-1.5 cm from the edge of geometrical field size. The central axis dose is higher at any depth. However, in a linac x-ray 
beam the beam profile exhibits higher dose or "horns" near the surface in the periphery of the field. These horns are created due to the flattening filter overcompensating near the surface to get a flat beam profile at greater depth, usually at $10 \mathrm{~cm}$. The penumbra is the region, near the edges of radiation beam, in which the dose falls off rapidly as a function of lateral distance from the beam axis.

There are three types of penumbra: geometrical penumbra, transmission penumbra, and scattering penumbra. Geometrical penumbra occurs due to the finite size of the source. Transmission penumbra is due to the transmission of radiation through the edges of the collimators. Transmission penumbra increases with increase in opening of the collimators because of the greater obliquity of the rays at the edges of the blocks. However, geometrical penumbra is independent of the collimator size. The scattering penumbra occurs because of the scattering of the radiation inside the patient. The combined effects of geometric penumbra, transmission penumbra, and scattering penumbra produces the dose variation along the field border. Dosimetrically, the physical penumbra width is the lateral distance between two specified isodose levels (eg. 80\% - 20\%) at a specific depth. Umbra is the region outside the radiation field, far from the edge. Dose in this region is very low and is the result of the side scatter from the field and radiation leakage from the collimator system.

\subsection{Intensity modulated radiation therapy (IMRT)}

The goal of radiotherapy is to maximize the tumor control while minimizing side effects. Tumor control requires delivery of a sufficient dose to the tumor, however excessive dose to the healthy tissue increases the normal tissue complication probability(NTCP). One way to reduce the normal tissue complication is to reduce the delivered dose, but this may decrease the tumor control probability (TCP). Therefore delivering the desired dose is a balancing act between TCP and NTCP.

Another way to spare the healthy tissue while delivering the desired dose to the 
tumor is to block the radiation outside the tumor as much as possible. In conventional radiotherapy, radiation outside of the target volume is blocked using collimators which have square or rectangular apertures [19]. However this may not completely spare the healthy tissue as the beam shape may not match the shape of the tumor.

Improvement in the conformality of the radiation delivery was introduced by $3 \mathrm{D}$ conformal radiotherapy (3DCRT) where the shape of the beam is matched to the shape of the target volume by using multi-leaf collimators (MLC). In this approach the beam intensity is uniform throughout the open field. Since the beam shape matches the tumor shape the healthy tissue sparing is greatly improved as compared to conventional radiotherapy. However, in a situation where the tumor is along the same beam path as a critical organ such as the spinal cord it is difficult to deliver radiation to the tumor only while sparing critical organs. Therefore the technique to deliver radiation beam needs further improvement.

Intensity Modulated Radiation Therapy (IMRT) is a more advanced innovation in radiation therapy. In this approach not only the beam shape matches the tumor shape but the fluence is modulated for each beam as shown in Figure 1.8(b). This makes it possible to reduce the dose in the areas near the sensitive organ and escalate the dose in areas away from the sensitive organ. So a desired dose distribution can be produced to match the complex/concave shape of the target [20] with a sharp dose gradient near the target and sensitive organ. Therefore dose conformity and healthy tissue sparing in IMRT is much higher compared to other methods. However IMRT is more sensitive to set-up errors and motion of the internal organs and it also takes longer time to deliver compared to 3DCRT.

Two approaches are used to modulate the beam intensity: filter-based IMRT and MLC-based IMRT. In filter-based IMRT, the intensity is modulated by placing a physical filter (compensator) in the path of beam. Linear accelerators without MLCs can deliver an IMRT plan using compensators which are fabricated with a computer-controlled 


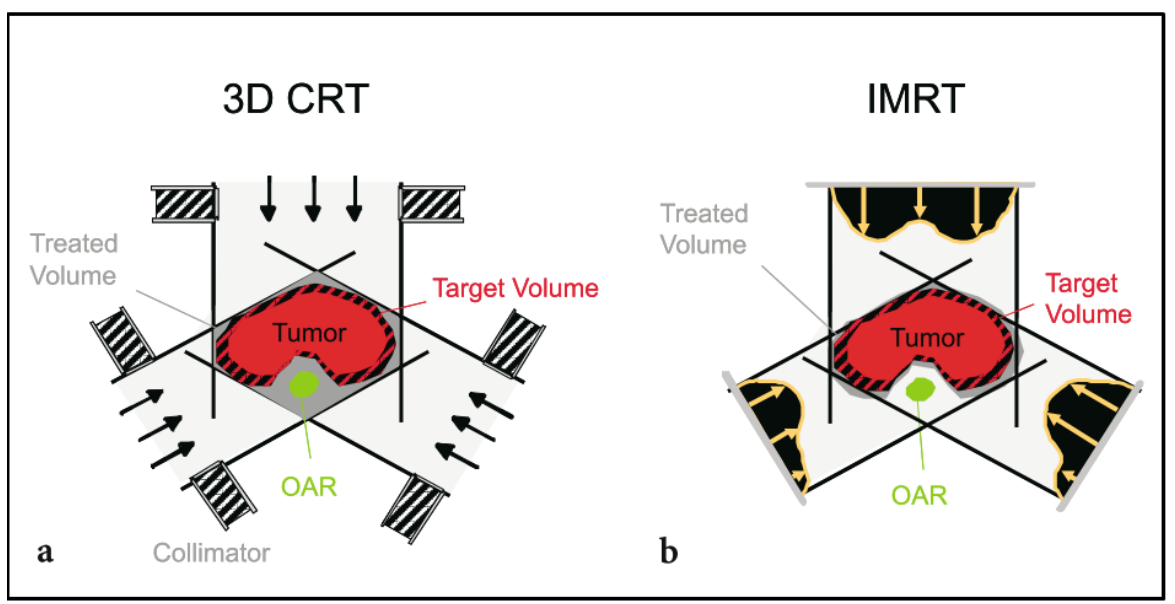

Figure 1.8: Illustration of beam intensity profile in 3DCRT (a) and in IMRT (b) [5].

milling machine. The disadvantage of this approach is that compensators need to be fabricated on a beam and patient specific basis, which is time consuming and costly. In MLC-based IMRT, the beam intensity is modulated by changing the beam aperture during delivery of a beam. Computer controlled MLCs are used to change the beam aperture. MLC-based IMRT is delivered either in static field delivery mode or in dynamic field delivery mode.

In static field delivery, the MLC leaves only move when the radiation beam is off and remain stationary when radiation beam is on. The treatment plan consists of several apertures or segments. Segments are shaped and delivered by the MLC; one at a time in a sequence without operator intervention. The superposition of different segments creates a modulated intensity pattern. This IMRT delivery method is called the step-and-shoot method $[2,21]$.

In the dynamic delivery mode, opposing pairs of MLC leaves move simultaneously in the same direction while the radiation beam is on. The velocities of the leaves determines the delivered fluence. This method is called dynamic MLC-based IMRT because modulation is done while the beam is on.

There are different approaches to IMRT treatment planning. "Forward" planning is 
similar to conventional planning in that the segment weights and shapes are adjusted manually by the planner based on their intution and experience. This method works well for tumors of simple shape that are not surrounded by numerous critical structures. The effectiveness of this method is limited for complex tumors geometries. Advantages of this method include the delivery efficiency and simplicity [22].

In an inverse planning approach, the inverse planning engine optimizes the beam apertures and fluence pattern in order to best meet the prescribed treatment objectives. Unlike in forward planning where beam apertures and intensities are manually optimized in few steps and resulting dose is compared to desired dose distribution, in inverse planning an optimization engine optimizes the aperture and intensities based on the prescribed dose and constraints in many iterative steps.

There are two approaches to IMRT optimization. In fluence-based inverse planning, each beam aperture is divided into small pencil beams known as bixels. Similarly, the treatment volume (CT cube) is divided in to small volume elements known as voxels. Since each pencil beam contributes to each voxel, the total dose to a voxel $i$ is the sum of dose from each bixel $j$ weighted by the corresponding bixel weight (intensity) $w_{j}$. Thus the dose in each voxel is given as [23]

$$
d_{i}=\sum_{j} D_{i j} w_{j}
$$

where the dose contribution matrix $D_{i j}$ is the dose delivered to voxel $i$ by bixel $j$ of unit weight. The dose $d_{i}$ is optimized by optimizing the bixel weights $w_{j}$, and the result is an optimized intensity (fluence) map. After the optimization is complete a leaf-sequencing algorithm translates the final intensity map for each beam into a set of deliverable beam segments [24].

Although fluence-based optimization is the most widely used approach to IMRT, it has some limitations. In fluence-based optimization the constraints imposed by the MLCs are ignored during intensity optimization. These constraints are considered only 
during the leaf-sequencing steps. The consequence is that the optimized intensity map can result in a large number of small segments. A large number of segments can lead to large leakage dose to the patient, long treatment time, and increased maintenance cost for MLCs. This problem is addressed in direct-aperture-optimization (DAO), where aperture shapes (segments) and weights are optimized simultaneously, and MLC constraints and number of segments are included in the optimization thereby eliminating the need for the separate leaf-sequencing step. The key feature of this method is that the user can define the number of segments to be delivered as a constraint during the optimization. The optimization only considers aperture shapes that satisfy the conditions set by the MLC. As a result, high-quality IMRT treatment plans can be generated using fewer segments (apertures) per beam. [24,25].

\subsection{Characteristics of respiratory motion}

The role of the lungs is to exchange $\mathrm{O}_{2}$ and $\mathrm{CO}_{2}$ between blood and air through a process known as respiration. The lung are situated inside the thoracic cavity. The most important muscle used in respiration is the diaphragm which separates the thorax from the abdomen. During inspiration, the diaphragm contracts and moves downward. This causes an increase in volume of the thoracic cavity. During normal breathing the lung volume changes up to 20\% [26]. Giraud et al. [27] reported that the maximum amplitude of diaphragm motion can be up to $67.8 \mathrm{~mm}$ in the superior-inferior (SI) direction with a mean amplitude of $34.3 \pm 20.4 \mathrm{~mm}$ between inspiration and expiration. Similarly, Hanley et al. [7] reported that the amplitude of diaphragm movement varies from 20 to $38 \mathrm{~mm}$, and the amplitude of chest wall movement from 2 to $2.5 \mathrm{~mm}$ during free breathing.

Some of the organs that move during breathing are the lungs, esophagus, liver, breast, kidneys, and prostate. However, the lung moves the most compared to other organs. So the motion effect will be highest for tumors in lung. Steven et al [28] studied 22 lung cancer patients with an average tumor size of $5.5 \pm 3.1 \mathrm{~cm}$ located in different parts of 
the lung. Among them, 10 patients showed no tumor motion in the superior-inferior (SI) direction, while the remaining 12 showed tumor motion with an average amplitude of $8.3 \pm 3.7 \mathrm{~mm}$ (range $3-22 \mathrm{~mm}$ ). They also found that respiration dependent lungtumor motion is independent of tumor size, tumor location, and pulmonary function test results. Similarly, Seppenwoolde et al [29] measured lung tumor motion in 20 patients using a fluoroscopic real-time tumor tracking system. They found that tumor motion was greatest $(12 \pm 2 \mathrm{~mm})$ in the SI direction for tumors situated in the lower lobes and not attached to the chest wall or vertebrae. Tumors attached to chest wall moved only slightly. The time-averaged tumor position was found to be close to exhale as the tumor spent more time in exhale than in inhale. Tumor motion was sinusoidal in the majority of patients. About fifty percent of the tumors followed a different path during inspiration and expiration showing hysteresis in the trajectory. The separation of the inhale and exhale trajectories was from 1-5 mm. In a study by Hanley et al. [7] the maximum amplitude of motion during free breathing was reported to be $20 \mathrm{~mm}$ for tumor in right mid lobe, and $15 \mathrm{~mm}$ for tumor in left lower lobe .

Characteristics of lung tumor motion can also be found in a paper by Shirato et al. [30]. In normal tidal breathing, the amplitude of motion was large (12 \pm 6$) \mathrm{mm}$ in SI direction for tumor in lower lobe not attached to chest wall. Motion of upper lobe tumors or tumors attached to rigid structures was $2.2 \pm 2 \mathrm{~mm}$. Motion in the leftright (LR) direction was $(1.2 \pm 0.9) \mathrm{mm}$ and in anterior-posterior (AP) direction was $(2.2 \pm 1.9) \mathrm{mm}$. The average period of respiration was $3.6 \pm 0.8 \mathrm{~s}$. No correlation was found between amplitude of tumor motion and patient characteristic such as height, weight, TNM stage, and pulmonary function.

From this literature review it can be inferred that diaphragm and lung tumor motion varies widely. Tumors in the lower lobe not attached to chest wall move the most. Lateral or AP motion is small compared to the motion in the SI direction. Also, the tumor spends most of the time in exhale and the tumor trajectory may have hysteresis. The amplitude 
and the trajectory of motion cannot be predicted from tumor size, location, and patient characteristics. This analysis suggests that tumor motion should be assessed individually for lung cancer treatment.

\subsection{Effects of motion}

Respiratory motion effects hinder the goal of the radiation therapy by compromising treatment delivery and the quality of images used to delineate the tumor. In the following sections the effects that occur if an organ moves during image acquisition or treatment are described [31].

\subsubsection{Effect on imaging}

During radiotherapy tumor information such as size, shape, and position is obtained through imaging. If tumor motion is not accounted for in image acquisition, it creates motion artifacts in the image. For example, in 3D CT which is generally used in conventional radiotherapy treatment planning, organ motion is not accounted for during image acquisition. This means that an image slice acquired in one phase of respiration is combined with another image slice acquired in a different phase of respiration creating a 3D image that is not a true representation of tumor (Fig 1.9). An ideal situation would be if all the slices acquired during imaging were in the same respiration phase. This happens only if the patient holds their breath or the acquisition is gated. But in reality, the tumor in the thorax and the abdomen are in motion due to respiration of the patient. One way that the tumor motion problem during imaging can be addressed is by using 4D CT in which tumor motion is accounted for during imaging. 4D CT is explained in section 1.9 . 

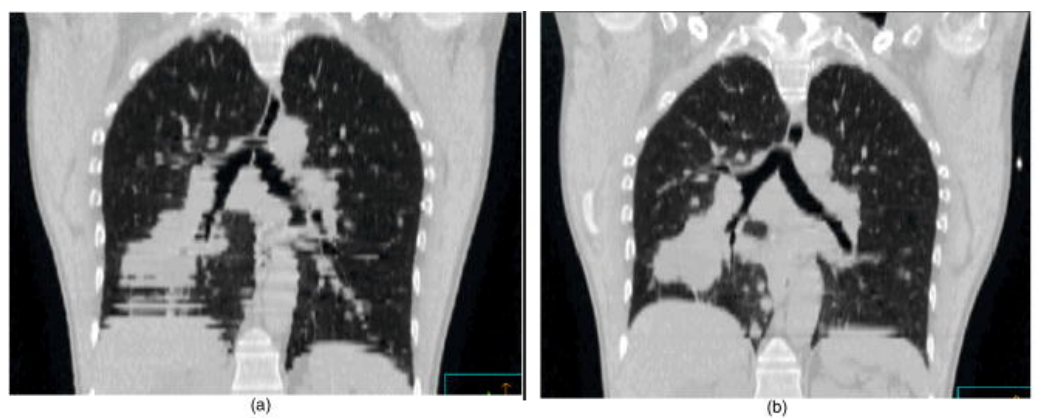

Figure 1.9: Coronal view of the CT scans of the same patient taken (a) during free breathing and (b) with respiratory gated scan at exhale. CT taken during free breathing has motion artifacts whereas respiratory gated CT shows the improvement in the image quality [26].

\subsubsection{Dose blurring}

Tumor movement during the treatment leads to geometrical mismatch between the radiation beam and the tumor location. The total dose at any point inside a moving organ is the average of the dose it receives over its range of motion, which is different from the static dose. The effect is more pronounced for a point near the edge of the tumor because part of the tumor volume moves in or out of the radiation field [32]. But for a point deep inside the target the effect is not significant because (1) the total dose from all treatment fields is almost uniform, and (2) the treatment period is much longer than the breathing period of patient [33]. Thus the dose distribution in the region near the border is blurred. The effect is independent of the delivery technique meaning that it occurs both in static and dynamic beam delivery. Intra-fraction and inter-fraction motion both cause dose blurring. Intra-fraction movement causes the dose distribution to be blurred in each treatment fraction, while the effect of inter-fraction motion can be seen only in the total cumulative dose. If there is inter-fraction motion but no intra-fraction motion the dose delivered in each fraction looks sharp but the total accumulated dose of all the fractions will be blurred. The blurring leads to large penumbra at the field edge making dose distribution less conformal as shown in figure 1.10 [31]. The dose blurring occurs 
not only due to organ motion but also due to set-up error if the set-up error is random.

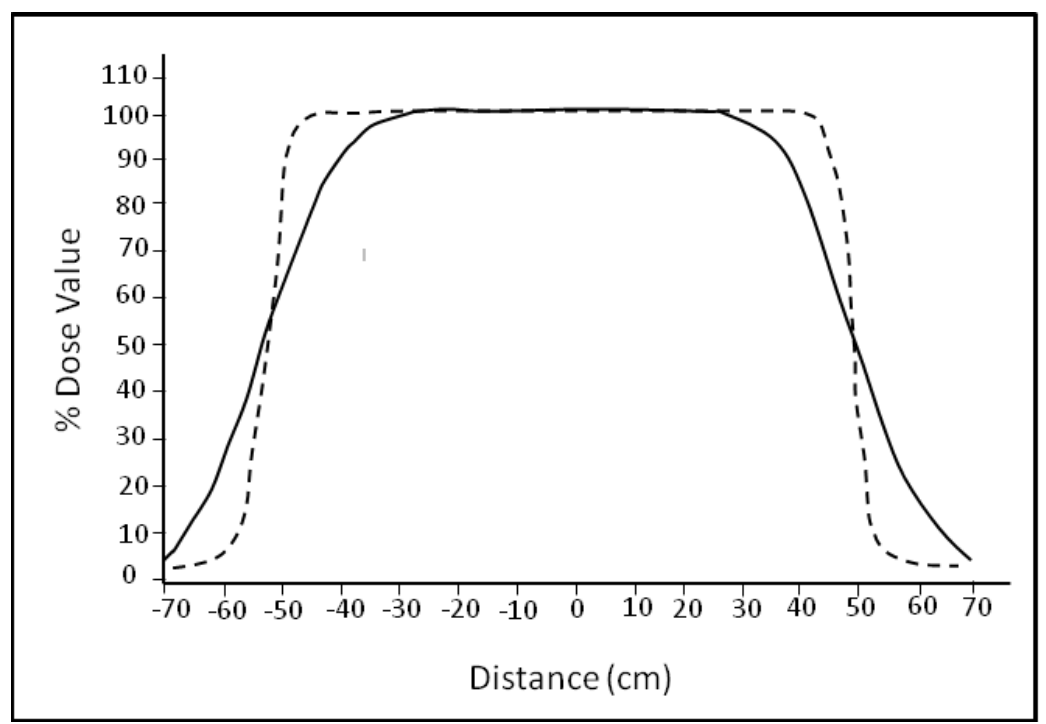

Figure 1.10: Illustration of dose blurring due to organ motion relative to radiation field. Dotted curve represents static dose profile and solid curve represents moving organ dose profile.

\subsubsection{Interplay effect}

The interplay effect occurs only for a dynamic dose delivery technique such as dynamic MLC based IMRT [33]. This effect occurs due to unsynchronized motion of organ and MLC leaves. As discussed above, there is dose blurring due to organ motion. On top of that, if the radiation beam is changing during treatment there is the interplay effect between intra-fraction motion of the organ and the radiation beam defined by the MLC apertures. This makes the variation of the dose in each voxel of the blurred region of the target even larger.

Figure 1.11 illustrates the interplay effect [6]. The leaves of MLC are moving from left to right. The organ motion is perpendicular to the motion of leaves. The filled star represents a point in the tumor and is moving down. The open star represents a point in an organ at risk and is moving up. The filled star moving downward never receives 

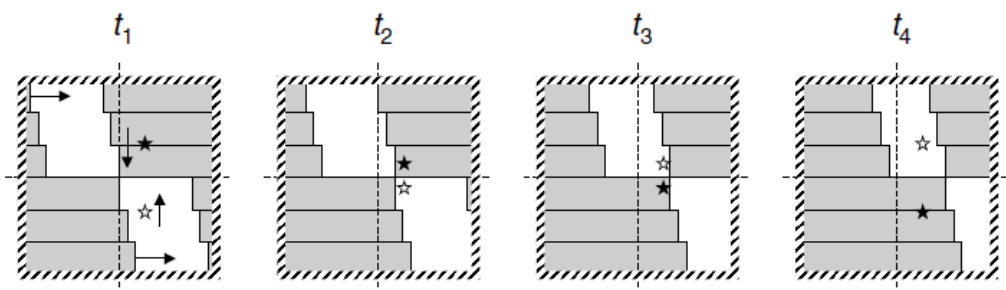

Figure 1.11: Illustration of interplay effect due to motion of organ relative to leafs of MLC [6].

primary dose between time $t_{1}$ and $t_{4}$, while the open star moving up receives primary dose at all time between $t_{1}$ and $t_{4}$. Seco et al. [34] reported that interplay effects are more pronounced if a beam is made up of small segments where the segment delivery time (1-2 sec) is of the order of breathing period (3-5 sec). For a single beam made up of a single segment the dose delivered to a point in 30 fractions can vary up to $20 \%$ and $10 \%$ for a medium sized segment. However, if the beam is made up of multiple small segments the variation in the delivered dose and motion-averaged dose to a point in the penumbra region is about only $2.4 \%$ - 4.3\% . For a beam made up of 100 segments, the error in the delivered normalized to motion-averaged dose is less than $1 \%$. Hence, either the increase in the segment weight or the increase in number of segments decreases the interplay effects.

\subsubsection{Dose deformation effect}

The dose blurring assumes that the dose distribution does not change as the anatomy moves with respiration, instead it moves with respect to the stable beam. This assumption is valid if the density of tissue is uniform (i.e medium is homogeneous). However, for a medium of non-uniform densities (heterogeneous media) the interfaces affect the dose distribution locally. If the interface moves there will be a distortion in the dose distribution. This effect is most pronounced for single fields and large amplitude motion. Bortfeld et al. [31] reported that the maximum effect of the distortion was $5 \%$ for 
(a)

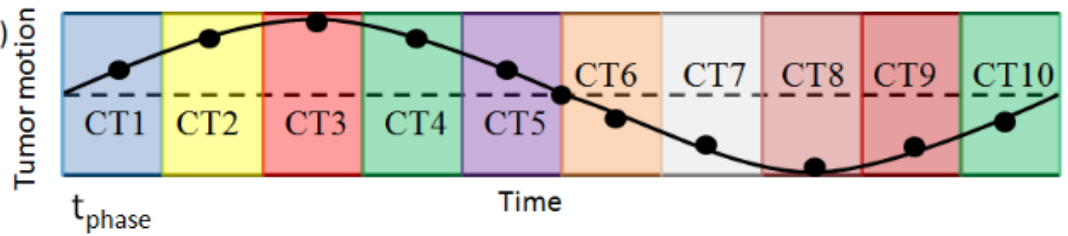

(b)

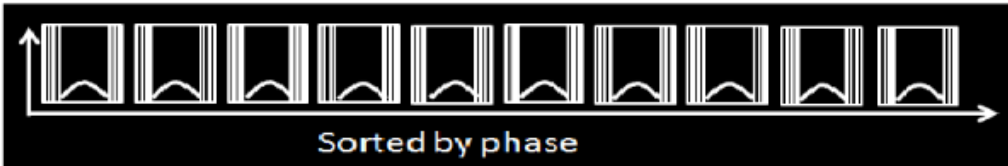

(c)

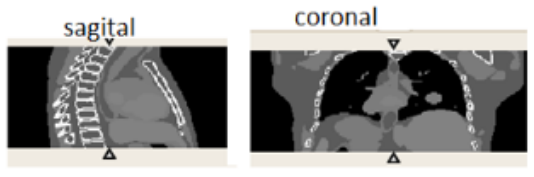

transverse

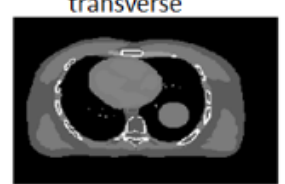

Figure 1.12: The mechanism of acquiring 4D CT images. (a) A breathing cycle of respiration is divided into 10 different phases. (b) CT image slices are stored in separated bins according to the phase in which it was acquired. (c) Combination of image slices in each bin form a 3D images of an organ in that phase.

a tumor displacement of $1.5 \mathrm{~cm}$.

\subsection{Respiratory motion information: 4D CT}

Tumor motion information may be obtained from a 4D CT image. 4D CT is a technique to acquire 3D images of a moving tumor. During 4D CT acquisition, tumor motion information is simultaneously measured and used to electronically tag each image slice as it is acquired. The recorded image slices are then sorted into different bins according to the phase in which they were taken. The image slices of each phase bin are combined together to form a 3D image of the patient's anatomy at that respiratory phase. A set of $3 \mathrm{D}$ images in different phases makes a 4D-CT data. The advantage of 4D CT over conventional 3D CT include reduced motion artifacts and the ability to image at different respiratory phases. Figure 1.12 shows the mechanism of acquiring 4D CT images. 


\subsection{Respiratory motion management techniques}

Uncertainty in the treatment planning and delivery arises from the patient setup error and variation in the size and shape of the CTV and organ motion. Setup errors can be reduced by immobilization techniques and careful positioning of the patient using portal imaging and alignment tools [35]. However, intra-treatment organ motion due to patient breathing presents a challenge in lung cancer treatment. As discussed above it creates artifacts in the simulation CT, compromises target coverage and increases the dose to the healthy tissue due to the mismatch between tumor location and the radiation beam. One approach to reduce the motion effect is to include the tumor motion information into the treatment planning processes so that we can either adjust the beam position or beam intensity or both. Motion information such as amplitude and characteristics of organ motion can be obtained from 4D CT images. Another approach is to minimize the respiratory motion amplitude during treatment delivery. Some of the approaches employed to manage the tumor motion during treatment are discussed below.

\subsubsection{Motion encompassing method}

In this approach, the PTV margin is designed by estimating the extent of setup uncertainty and organ motion. The ITV is estimated either from slow CT, inhale-exhale breath-hold CT or from 4D CT [26]. This approach is simple and results in good CTV coverage but increases integral dose to the patient substantially and critical tolerance dose of certain structures may be exceeded if they are close to the PTV [31].

\subsubsection{Breath hold}

In the breath-hold technique, the patient is coached to hold their breath at either full exhalation or at full inhalation for an extended period of time. Inhalation is favored as holding breath during inspiration increases lung volume and decreases lung density. From 


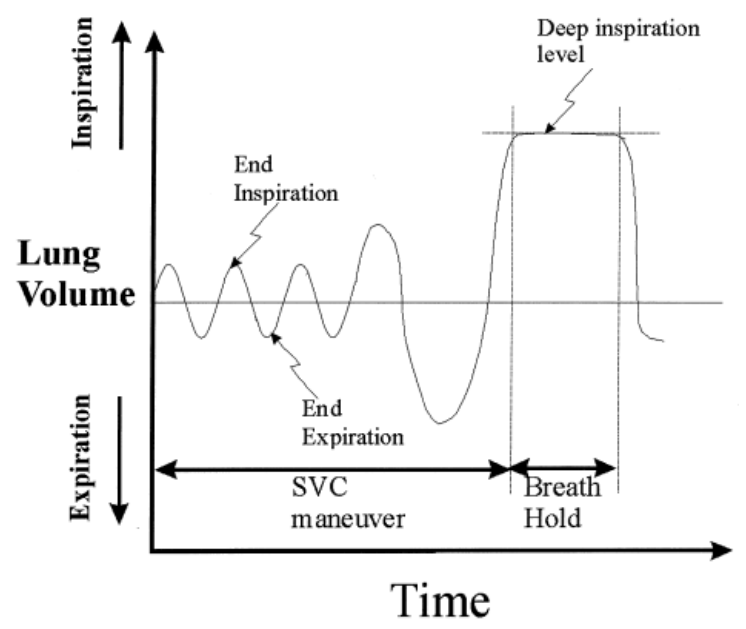

Figure 1.13: Graph shows that deep inspiration breath-hold increases the lung volume for extended period of time [7].

this situation we get two distinct advantages. First, the treatment margin is reduced due to immobilization of the tumor; second, the volume of normal tissue exposed to a high dose is decreased. An illustration of lung volume variation over a deep inspiration breath hold is shown in Figure 1.13. In a study by Hanley et al. [7] it was found that volume of lung receiving dose more that 25 Gy was reduced by $30 \%$ in deep-inspiration-breath-hold (DIBH) of 112-16 sec compared to free breathing. However, breath-hold requires patient training, and it is difficult for patients with poor pulmonary function.

Sometimes the patient is assisted to hold their breath using an 'active breathing control' device. In this technique, a nasal clip is used to prevent nasal breathing, forcing the patient to breath through the mouth. The patient is forced to hold their breath temporarily by blocking the air flow both in inspiration and expiration at predefined volumes that are monitored by spirometry. For stereotactic irradiation of early stage lung cancer treatment a technique known as 'forced shallow breathing with abdominal compression' is used [26]. The technique employs a stereotactic body frame with an attached plate that is pressed against the abdomen. The applied pressure to the abdomen reduces diaphragm excursion thereby reducing the tumor excursion. 


\subsubsection{Respiratory gating}

In this approach, the patient is allowed to breathe freely, and the radiation beam is turned on only for a small portion of the respiration cycle known as the gating window. Respiration is monitored either by detecting the movement of an external marker placed on the abdomen or by detecting the signal from an internal fiducial marker placed in or close to the tumor [36]. When the signal is within the gating window the beam turns on automatically. At all other times the beam is off. Usually the gating window is chosen near exhale. In one study it was found that the volume of lung receiving dose more than 25 Gy was reduced by $18 \%$ [7] in gated respiratory technique compared to free breathing. Although gating increases dose conformation and healthy tissue sparing, the drawback is that it increases the treatment time significantly.

\subsubsection{Real time tumor tracking}

In this approach the tumor position is tracked in real time and the beam position is adjusted dynamically. There are two approaches to track the tumor in real time. One approach is to adjust the opening of the multi-leaf collimator to track the real-time tumor position. The tumor position in real time is measured by detecting the gold markers implanted in or near the tumor using a fluoroscopic real time tumor tracking system [37]. Other approaches to tumor tracking include using a Cyberknife [38] which is a compact linac mounted in a robotic arm. Another approach is to use a robotic couch [36]. In this approach the couch position is adjusted according to the tumor position as detected by the tracking system, while the linac remains stationary. As in gating, real time tumor tracking increases the dose conformation. However, the draw back is that it significantly complicates the treatment procedure [23]. 


\subsubsection{D optimization}

The approaches discussed above either increase the treatment beam size, constrain the tumor motion by altering the patient breathing, or follow the tumor motion. These methods either compromise the healthy tissue sparing, and/or increase the treatment time and complexity of the procedure. 4D optimization is another approach to achieve a highly conformal dose delivery while allowing the patient to breathe freely. The idea behind this approach is to incorporate the tumor motion information into the treatment plan, therefore deconvolving the blurring effect of the motion.

Trofimov [23] outlines four different 4D optimization approaches to make a plan optimal for a moving tumor. In all approaches motion information is obtained from 4D-CT data. In each of the approaches discussed below, an optimization similar to conventional static IMRT optimization is performed, however, they differ in the way the motion information, dose calculation, and beam intensity map are combined and utilized.

1) Motion pdf approach: To adapt the motion information into treatment planning, the tumor displacement during one respiration cycle is divided into a number of phases. The pencil beam dose distributions are calculated for each phase and then mapped back to the reference phase and weighted by motion pdf to obtain a ' $4 \mathrm{D}$ ' pencil beam dose distribution $[39,40]$. If the dose a voxel $i$ receives in the given phase $x$ is

$$
d_{i}(x)=\sum_{j} D_{i j}(x) w_{j}
$$

then the total dose it receives in all phases of respiration is

$$
\left\langle d_{i}\right\rangle=\sum_{x} \sum_{j} D_{i j}(x, 0) w_{j}
$$

where $D_{i j}(x, 0)$ is the dose matrix mapped to the reference phase. However, to make the plan optimal for a given breathing pattern, the motion information is introduced by 
the probability distribution function (pdf) of tumor motion. For this, the dose a voxel receives at any phase is weighted by probability of it to be in that phase.

$$
\left\langle d_{i}\right\rangle=\sum_{x} \sum_{j} D_{i j}(x, 0) p(x) w_{j}
$$

where $p(x)$ is the fraction of time spent in phase $x$. The pencil beam weights $w_{j}$ are optimized so that the dose $\left\langle d_{i}\right\rangle$ meets the plan objective. This motion pdf approach is effectively the deconvolution of the dose blurring effect.

2) Optimal gating: In optimal gating, the gating window is optimized to encompass the phases with the least amount of motion [41]. For this, dose matrices are calculated on each phase and mapped to reference phase as in the motion pdf approach. The difference between optimal gating and pdf approach is that in optimal gating only certain phases are chosen that make window optimal. In this manner several plans are optimized for gating on different windows, and the best one is chosen for delivery.

3) Optimal tracking: In this approach more dose is delivered to phases in which the treatment objectives are easily achievable, e.g when separation between the target and critical structures is maximum. The delivered dose distribution in different phases of motion will not be same. However, the sum of doses adds up to a desired dose distribution [23]. For a optimized tracking plan dose contribution matrices are calculated for different respiration phases and are mapped to reference phase. Then the optimization is done for each phase simultaneously.

$$
\left\langle d_{i}\right\rangle=\sum_{j} D_{i j}(1,0) p(1) w_{j}(1)+\sum_{j} D_{i j}(2,0) p(2) w_{j}(2)+\cdots
$$

or

$$
\left\langle d_{i}\right\rangle=\sum_{x} \sum_{j} D_{i j}(x, 0) p(x) w_{j}(x)
$$

where $w_{j}(x)$ is the intensity map for phase phase $x$ and $p(x)$ is the corresponding phase 
duration. The difference between this approach and other approaches discussed above is that the other approaches generate only a single of optimized intensity map for each beam. However, this optimal tracking approach results in different intensity maps for each phase, i.e $w_{j}(x)=\left[\left(w_{1}(1)+w_{2}(1)+..\right),\left(w_{1}(2)+w_{2}(2)+..\right), \ldots . .,\left(w_{1}(x)+w_{2}(x)+..\right)\right]$. These set of intensity maps are then used to generate dynamically changing 'tracking' MLC segments for delivery.

\subsection{Motion variations}

Although the 4D optimization methods discussed above account for the tumor motion in the planning, they ignore the uncertainties in the tumor motion. These approaches are based on the assumption that tumor motion is stable and reproducible during treatment delivery. However, tumor motion is neither stable nor reproducible. Variation in tumor motion arising from the variations in breathing pattern degrades the quality of dose distribution making the plan suboptimal. A patient's breathing pattern may vary in magnitude, period, regularity, and/or in baseline shift. Such variation may occur in an inter-fraction basis as well as intra-fraction basis. Shirato et al. [30] have reported $12 \pm 6 \mathrm{~mm}$ of changes in mean amplitude of tumor motion during normal tidal breathing, and 2.7 to $6.6 \mathrm{sec}$ of changes in the breathing period. Similarly, variations in breathing pattern are reported in a study by Keall et al. [26]. Figure 1.14 shows the variation in breathing traces measured in a few minutes apart. In (a) the breathing pattern is relatively reproducible in shape. However in (b) breathing traces are so irregular that it is difficult to distinguish any pattern.

Zhang et al. [42] have evaluated the inter-fraction variations of lung tumor motion using motion pdfs. Figure 1.15 shows such pdf variations of a representative patient over different treatment fractions. From these literature reviews it can be inferred that tumor motion not only differs between patients but also changes during the treatment of a patient introducing uncertainties in the treatment planning. 


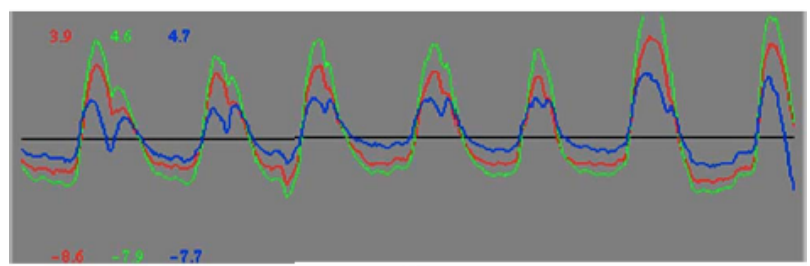

(a)

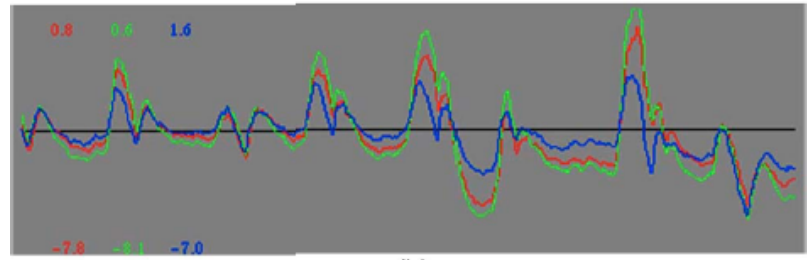

(b)

Figure 1.14: The respiratory pattern variation of a patient within a few minutes apart. Three curves in different colors represent surrogate motion in SI, AP and LR direction $[26]$.
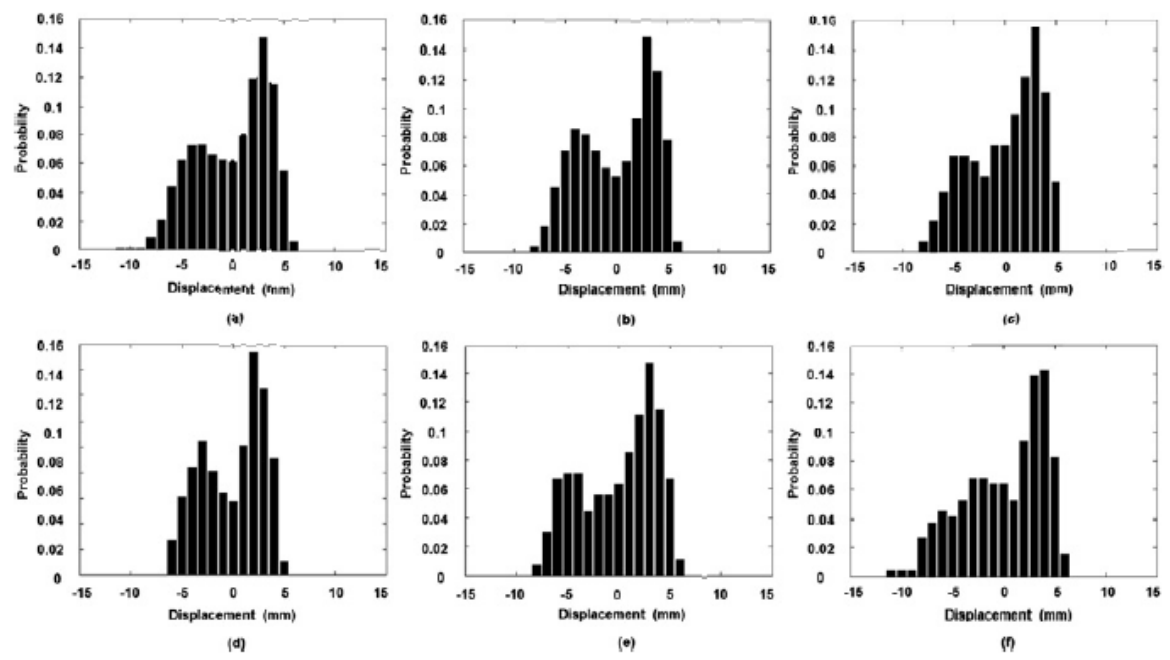

Figure 1.15: The motion pdf variation of a representative patient in different treatment fractions. (a) is average pdf, and (b)-(f) are pdf for different treatment fractions [42]. 


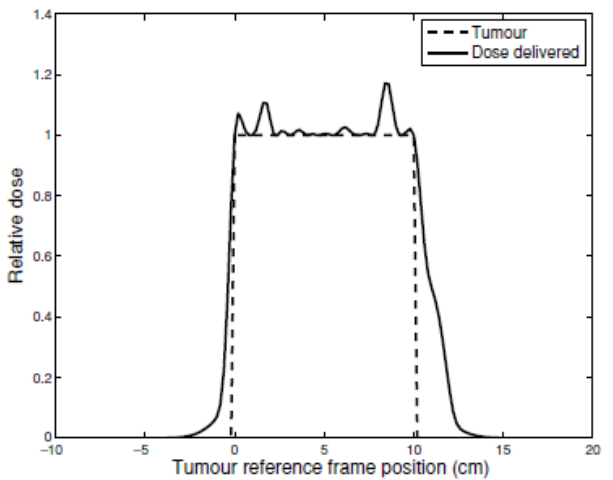

(a)

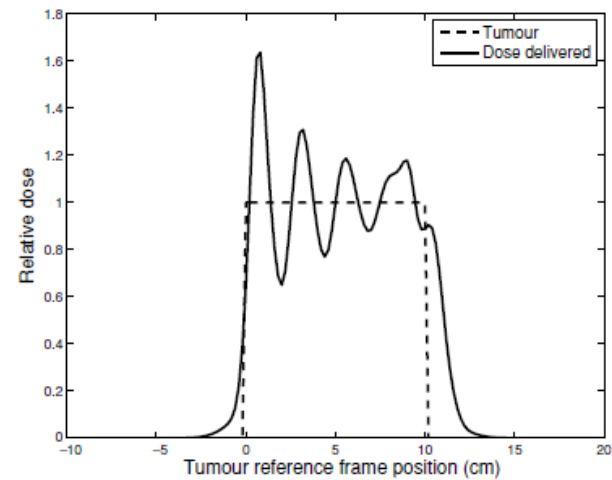

(b)

Figure 1.16: Effect of motion uncertainty in the treatment. (a) Dose profile when tumor motion during treatment delivery is same as in planning. (b) Dose profile when tumor motion during treatment delivery is different from planning [40].

Chan et al. [40] has analyzed the effect of motion uncertainties on a 4D optimized treatment plan. They have shown that a treatment plan optimized for moving tumor achieves treatment goals if the tumor motion during treatment delivery is same as during planning (Fig.1.16) (a). However the plan becomes suboptimal when tumor motion during treatment delivery is different from the tumor motion used in the treatment planning (Fig.1.16 (b)). The effect of motion uncertainties can also be found in a study by Heath et al [43]. They report that if the variations in amplitude and/or baseline position are not included in the treatment planning there is a large variation in the delivered dose to the target. But variation in delivered dose is highly reduced if the motion variations were accounted for in the planning. These studies lead us to the conclusion that there should be an approach that not only considers the tumor motion but also address the variations in tumor motion to make the plan robust under such circumstances. 


\subsection{Robust optimization}

Robust optimization is an approach to address the uncertainties in the tumor motion during treatment. One approach to account tumor motion uncertainty is the probabilistic optimization as discussed by Heath et al. [43]. This approach employs a quadratic objective function with a variance term $V_{i}$ representing the variance of the dose in each voxel of the target volume, and the phase weighted cumulative dose $\left\langle D_{i}\right\rangle$. The objective function is

$$
\begin{gathered}
F_{\text {target }}=\sum_{i}^{N_{t}}\left[\alpha_{u}{ }^{t}\left(D_{\min }-\left\langle D_{i}\right\rangle\right)_{+}^{2}+\alpha_{o}^{t}\left(\left\langle D_{i}\right\rangle-D_{\max }\right)^{2}\right]_{+}+V_{i} \\
V_{i}=\sum_{i}^{N_{k}}\left[\left\langle D_{i}^{2}\right\rangle_{k}-\left\langle D_{i}\right\rangle_{k}^{2}\right]
\end{gathered}
$$

where $N_{t}$ is the number of voxel in the target, $N_{k}$ is the number of treatment beams, $\alpha_{u}^{t}$ is under dose penalty and $\alpha_{o}^{t}$ is over dose penalty. The cumulative dose which is the sum of dose a voxel receives in each phase weighted by the product of corresponding probability distribution of motion amplitude and baseline shift is

$$
\left\langle D_{i}\right\rangle=\sum_{\mu} \sum_{\eta}\left\langle D_{i}\left(A_{\mu}, b_{\eta}\right\rangle P\left(A_{\mu}\right) P\left(b_{\eta}\right)\right.
$$

and $P\left(A_{\mu}\right)$ and $P\left(b_{\eta}\right)$ are Gaussian probability distributions of motion amplitude and base line shift respectively.

Heath et al. [43] also proposed another method of optimization known as worst-caseoptimization. The objective function employed in worst-case approach is the sum of the objective function for the nominal dose distribution (no error) and the worst-case dose distribution weighted by the probability $\left(P_{w c}\right)$ of worst-case distribution.

$$
F=F\left(D_{n o m}\right)+F\left(D_{w c}\right) P_{w c}
$$




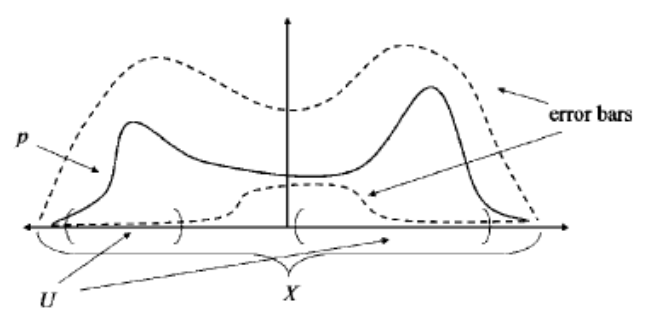

Figure 1.17: Model of uncertainty in motion pdf [40].

Chan et al. [40] and Vrancic et al. [44] have proposed a model of uncertainty in the motion pdf which, instead of using a variance term in the objective function, considers variations in the pdf during optimization. Their optimization model consists of a nominal pdf $p$ surrounded by upper and lower "error bars" $p+\bar{p}$ and $p-\underline{p}$ respectively. The nominal pdf is the average of the set of pdfs, and error bars bound the deviations of any pdf from above and below the nominal pdf as shown in Figure 1.17. In this approach the robust formulation for the target volumes $t$ is

$$
\begin{array}{r}
\text { minimize } \sum_{t} \sum_{i} \sum_{j} D_{t, i, j}(x, 0) p(x) w_{j} \\
\text { subject to } \sum_{i} \sum_{j} D_{t, i, j}(x, 0) \widehat{p}(x) w_{j} \geqslant d^{p r e s}
\end{array}
$$

where $w_{j}$ is the bixel weight, $d^{p r e s}$ is the prescribed dose, and $p(x)-\underline{p}(x) \leq \widehat{p}(x) \leq$ $p(x)+\bar{p}(x)$.

This robust optimization approache addresses the issue of motion uncertainty as demonstrated in Figure 1.18 which shows the invariability of the dose profile even if the treatment pdf is different from the planning pdf [40]. 


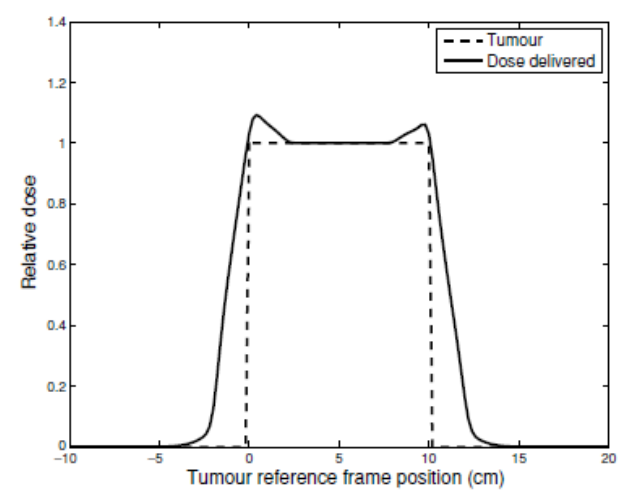

(a)

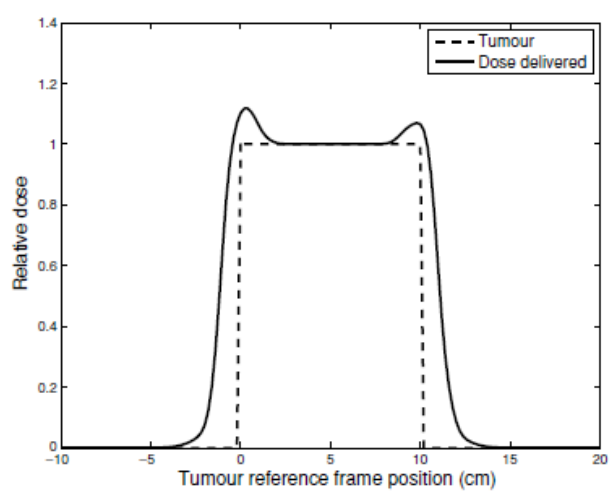

(b)

Figure 1.18: Dose profile in robust optimization using model of uncertainty in motion pdf when tumor motion during treatment delivery and planning is same (a) and different (b) [40].

\subsection{Hypothesis and objective}

Robust optimization has been demonstrated to address the issue of motion uncertainty. However, the methods of Chan and Heath were implemented in research treatment planning systems. The implementation of these models demand the modification in the existing treatment planning system requiring FDA approval. Hence the approach although being good for a research planning system is not applicable clinically. Further more these models require a large number of uncertainty sets to be sampled which makes the implementation of the plan computationally too intense. Therefore, we feel a need to find a simpler approach.

Our objective is to develop a robust optimization method that is clinically applicable in the existing treatment planning system with out any computational difficulty or need of modification in the existing system. We propose that these objectives can be achieved by the use of either (1) the average-pdf from the set of patient pdfs or (2) a set of "worst case" pdfs representing the largest variations in the optimizations formulation. Therefore our hypothesis is 
"A 4D plan based on the average-pdf or worst case pdfs is robust to any changes to tumor displacement"

To test our our hypothesis, we will optimize 4D plans using either the average-pdf or worst case pdfs from a set of simulated motion pdfs to make a robust plan. The robust plan will then be applied to each of the individual motion pdfs separately. If the dose distribution remains invariant with the change of pdf then the hypothesis is said to be valid. 


\section{Chapter 2}

\section{Materials and methods}

In this chapter the software used to generate image data and design treatment plans is described. The methods for generating robust treatment plans that account for respiratory motion are presented. A set of motion variation scenarios that were used to evaluate the robustness of these plans is described.

\subsection{Materials}

\subsubsection{XCAT phantom}

XCAT [8] is a virtual phantom software that can be used to generate 4D CT images. The advantage of the digital phantom is that the user can control parameters such as tumor size and motion amplitude.

XCAT provides a virtual model of patient's anatomy and physiology making it possible to do experiments entirely on the computer. It can realistically model complex shapes of human (both male and female) organs and has the flexibility to model anatomical variation and physiological motion. A model human anatomy generated by XCAT is shown in figure 2.1 [8]. Although the phantoms generated by XCAT are virtual they look very realistic. The software is very user friendly allowing the user to control organ motion, 


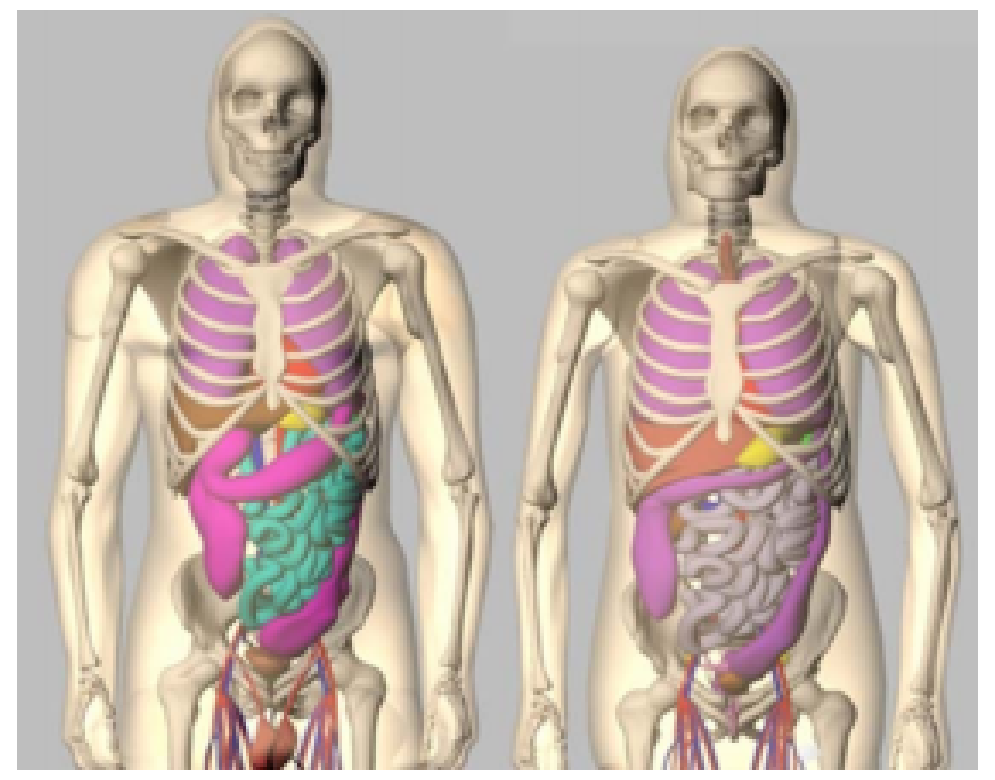

Figure 2.1: Anterior view of male (left) and female (right) anatomies of 4D XCAT phantom [8]

tumor size, and shape.

XCAT works in six different image modes. Mode 0 generates a phantom of the whole body (human torso). Mode 1 generates a heart lesion at a user defined location. The heart lesion is subtracted from the image generated in mode 0 to place a defect in the heart. Similarly, mode 2 generates a spherical lesion of user defined size at a user defined location. In this study we generated a spherical lesion with a diameter of $30 \mathrm{~mm}$ at the co-ordinates of $(\mathrm{x}, \mathrm{y}, \mathrm{z})=(215,270,42)$ which places it in the right lung when combined with the image generated in mode 0 . Mode 3 generates cardiac plaque. The phantom in mode 3 is combined with phantom in mode 0 to the place plaque in the heart. Mode 4 outputs phantom motion vectors in text format. These vectors give the organ movement due to heart beat and/or respiration. Mode 5 generates a phantom from user defined anatomy parameters.

There are three motion modes. Mode 0 models only heart motion, mode 1 models 
only respiratory motion, and mode 2 models both heart and respiratory motion. Using these modes, the user can choose whether to incorporate cardiac and/or respiratory motion into the phantom sets. We chose mode 1 to incorporate respiratory motion only. The user can also choose the length and the starting phase of both the heart and respiratory motion. Normal values for length of heart beat and respiratory cycles are 1 sec and 5 secs, respectively. An index value between 0 and 1 is used to specify the starting phase of the heart or respiratory cycles, for example if the starting phase is full exhale, the index is 0.0 , and if it is full inhale the index is 0.4 . Breathing motion can be altered by changing the respiration period, diaphragm motion, and/or chest wall motion.

The number of 4DCT phase images generated by XCAT can be controlled by choosing the number of output frames. We generated 10 frames per respiration period of $5 \mathrm{sec}$ starting at full exhale. Each of these CT image represents a different phase of a patient's respiratory cycle. Each images consisted of 80 slices with slice width $3.125 \mathrm{~mm}$, a pixel size of $1.5625 \mathrm{~mm}$ and a $512 \times 512$ field of view.

The image intensities generated by XCAT represent linear attenuation coefficients. Since the CT images used for treatment planning are in CT numbers (Hounsfield Units) we convert the XCAT image intensities into Hounsfield Units (HU) by the following formula

$$
H U=\frac{\mu_{x}-\mu_{w a t e r}}{\mu_{w a t e r}} \times 100
$$

where $\mu_{x}$ is the the attenuation coefficient of tissue and $\mu_{w a t e r}$ is the attenuation coefficient of water.

\subsubsection{Virtuos}

Virtuos [45] is a software for radiotherapy planning. It allows three dimensional visualization of the image, delineation of the volumes of interest, treatment plan design, dose calculation, and plan evaluation. It has four different working modes: image processing 


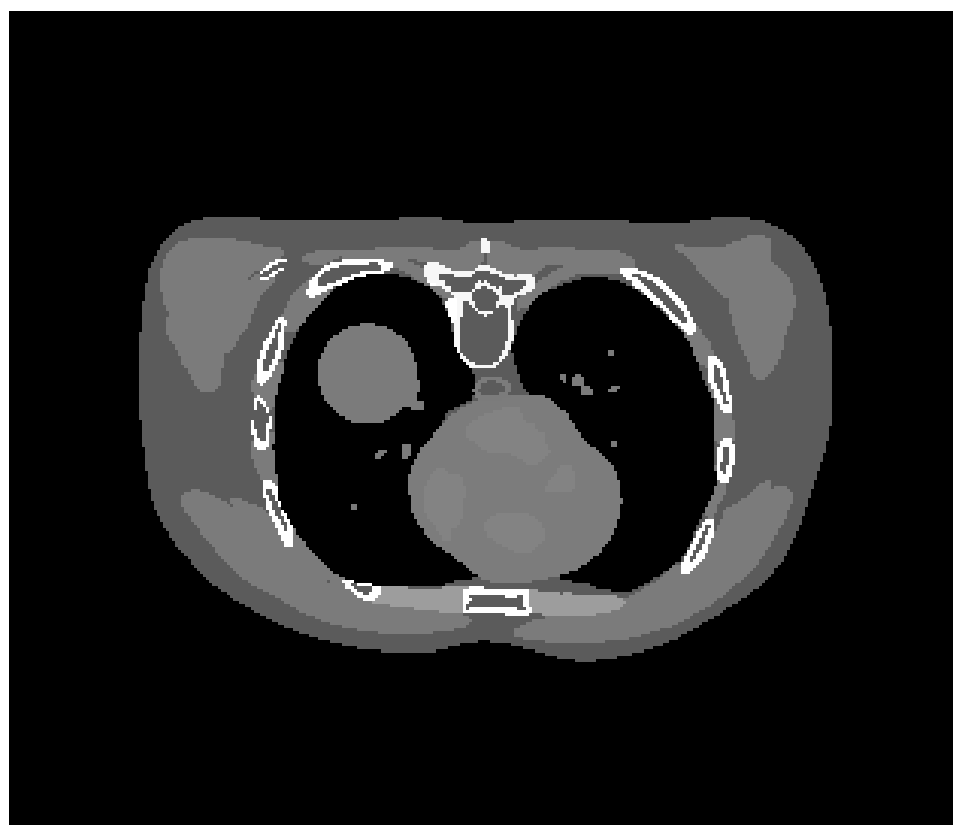

Figure 2.2: Reconstructed transverse CT slice simulated using XCAT phantom with a spherical lesion of diameter of $30 \mathrm{~mm}$ in the right lung.

mode, plan mode, result mode and compare mode. In image processing mode, the user can load different image cubes, register them, and delineate different structures. The delineation is done by drawing the outline of a structure in every transverse slice. Figure 2.3 shows the contours of the GTV, CTV, ITV, right lung, left lung, heart, spinal cord, and body.

After the delineation of the patient's anatomy, a treatment plan can be designed in planning mode. It allows the user to choose the desired number of beams and their incident angles. The treatment beam energy, and gantry angle can also be selected. For an intuitive visualization of treatment beams and treatment volume Virtuos provides 2D and $3 \mathrm{D}$ views of the beam placement on the patient. Figure 2.4 shows a treatment plan with 5 treatment beams at gantry angles of 0, 180, 225, 270, and 315 degrees.

In result mode, Virtuos allows the planner to evaluate the calculated dose distribution qualitatively and quantitatively by calculating dose volume histograms and statistics for 


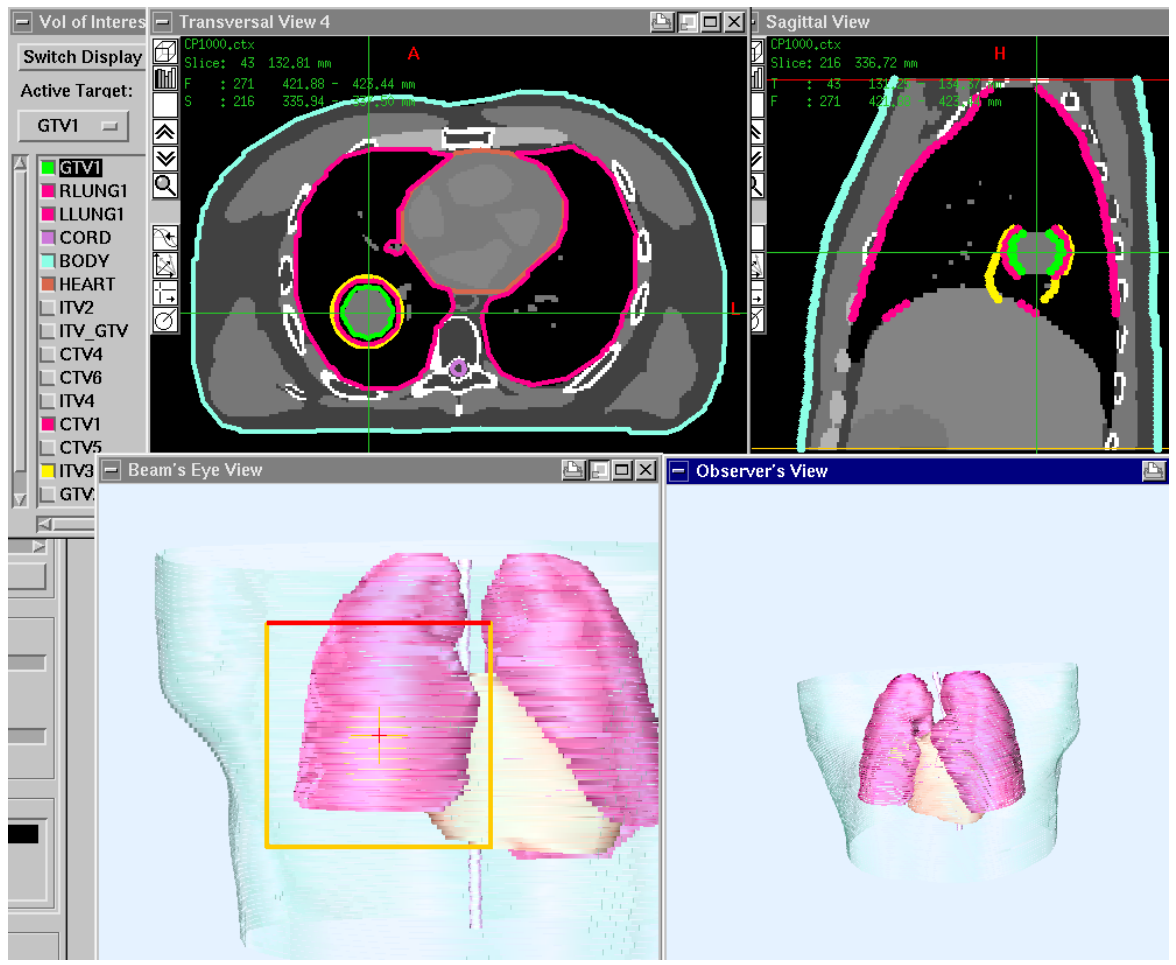

Figure 2.3: Virtuos image processing mode showing the delineation of different volumes of interest in transverse, sagital, beam's eye view and observer's view. Legend: GreenGTV, red-CTV, yellow-ITV, magenta-lungs, orange- heart, pink-spinal cord, blue- body.

different structures as shown in Figure 2.5. The dose distribution can be visualized from three different directions namely frontal view, sagital view, and traverse view.

\subsubsection{KonRad}

IMRT treatment plans designed in Virtuos were optimized using an inverse treatment planning system called KonRad [9], originally developed at the German Cancer Research Center (DKFZ). KonRad performs fluence-based optimization. The CT cube is divided into small volume elements called voxels, with a size of $2.62 \times 2.62 \times 2.62 \mathrm{~mm}^{3}$. Similarly, each treatment beam is divided into pencil beams known as bixels of size $5 \times 5 \mathrm{~mm}^{2}$. The relationship between bixels and voxels is shown in Figure 2.6. KonRad determines the optimal weight, $w_{j}$, of each bixel in order to satisfy, as closely as possible, a set of 


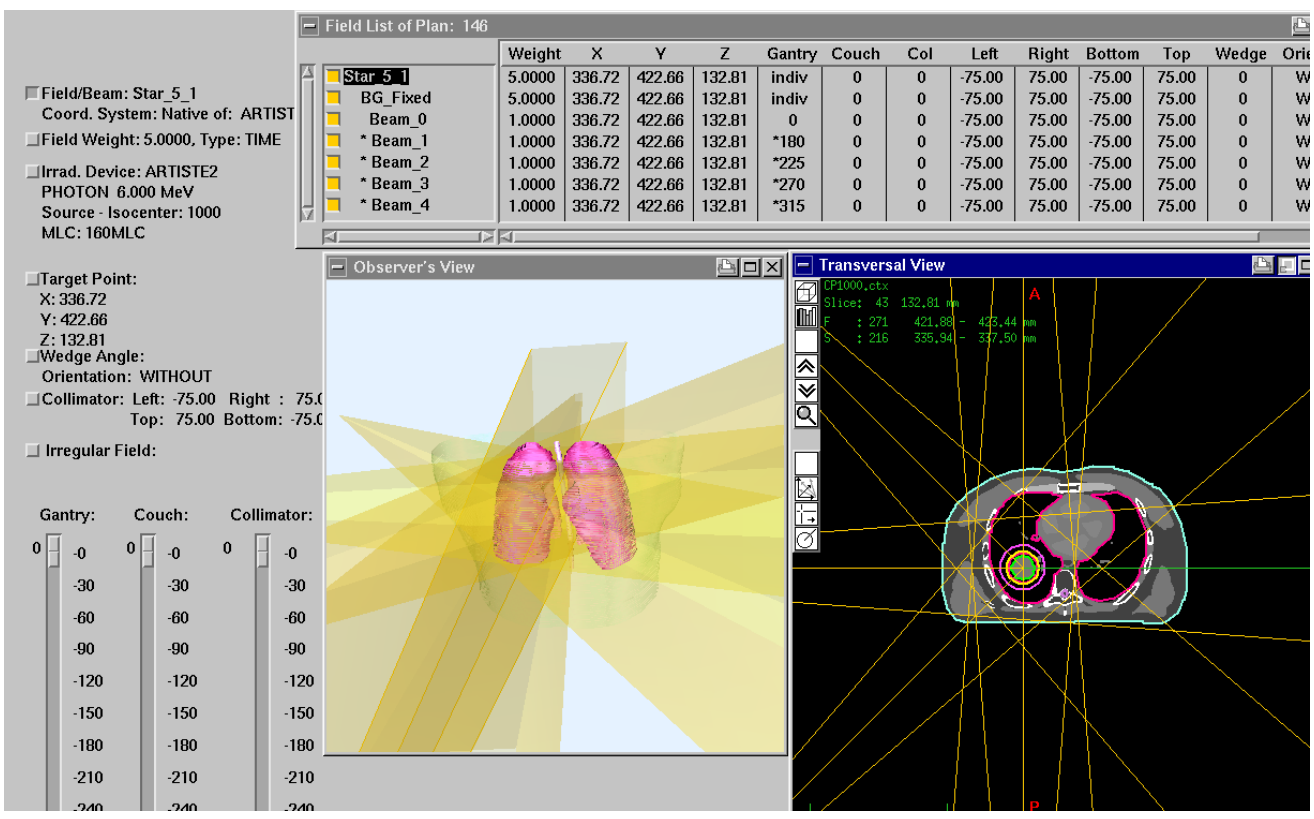

Figure 2.4: Virtuos in planning mode showing the 3D patient model in observer's view, and $2 \mathrm{D}$ transverse view of beams arrangements with different parameters such as beam energy and gantry angles.

user defined dose constraints.

In the first step of the optimization, the dose contribution of each bixel $j$ to each voxel $i$ is calculated. The matrix containing the dose contribution of all bixels in a single treatment beam is called the $D_{i j}$ matrix. The total dose $d_{i}$ delivered to voxel $i$ is the linear superposition of the dose delivered by all the bixels in each beam multiplied by their weights.

$$
d_{i}=\sum_{j}^{N_{j}} D_{i j} w_{j}
$$

where $w_{j}$ is the optimized weight of the bixel and $N_{j}$ is the number of bixels. The dose contribution matrix $D_{i j}$ is calculated only once. Once the $D_{i j}$ matrix is calculated, the optimization engine starts the optimization loop to find optimal bixel weights. If there are $N_{k}$ beams used in the treatment plan then the total dose in voxel $i$ is 


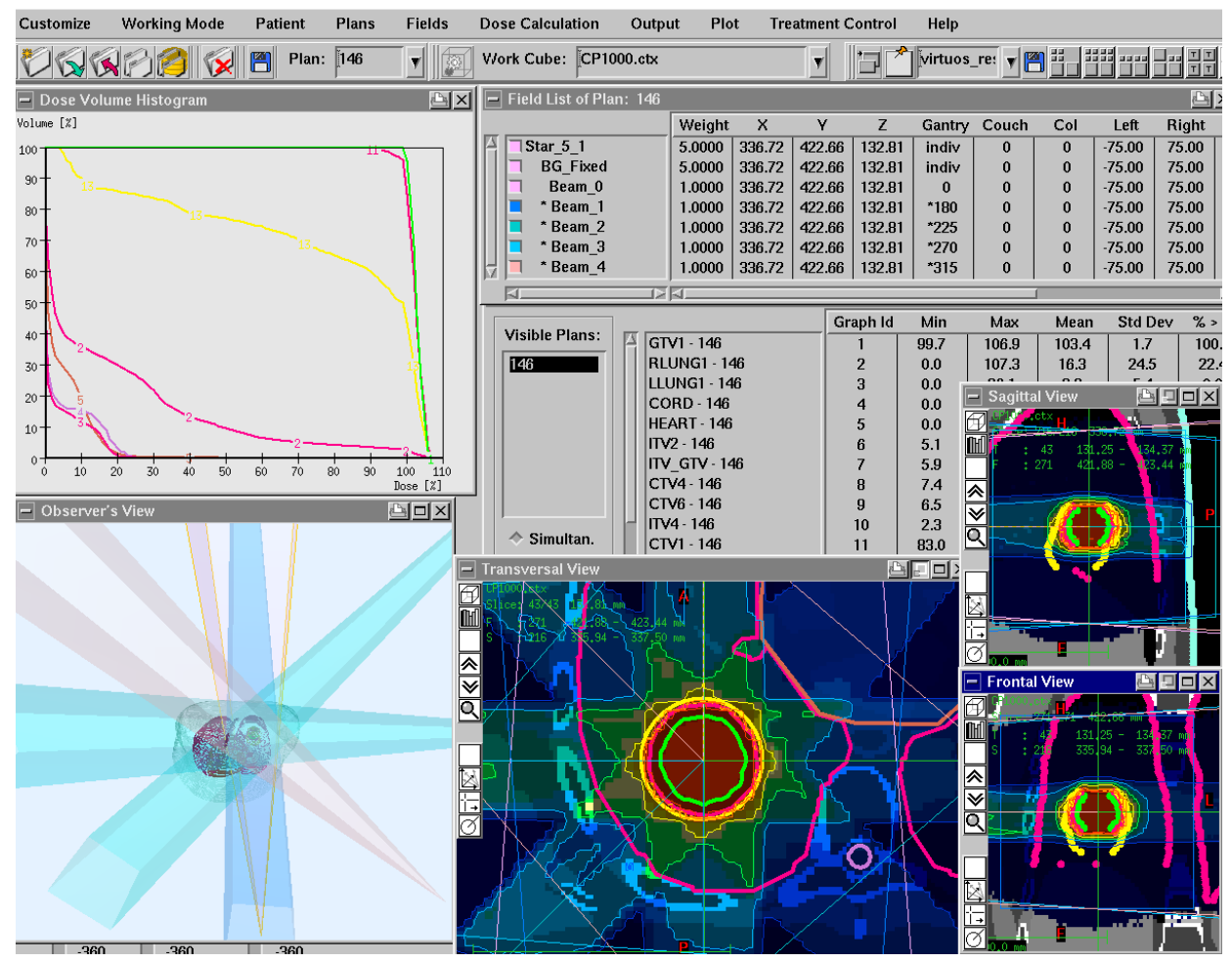

Figure 2.5: Virtuos in result mode allows to evaluate the the treatment plan by providing DVHs, dose statistics, and dose distributions.

$$
d_{i}=\sum_{k}^{N_{k}} \sum_{j}^{N_{j}} D_{i j}^{k} w_{j}^{k}
$$

\subsubsection{Optimization engine}

The optimization engine is responsible for the calculation of the bixel weights $w_{j}$. The optimization engine has two components: the objective function, and the optimization algorithm.

The objective function: The plan is optimized by determining the bixel weights which minimize the value of the objective function. We used the quadratic objective function which minimizes the difference between actual and desired dose 


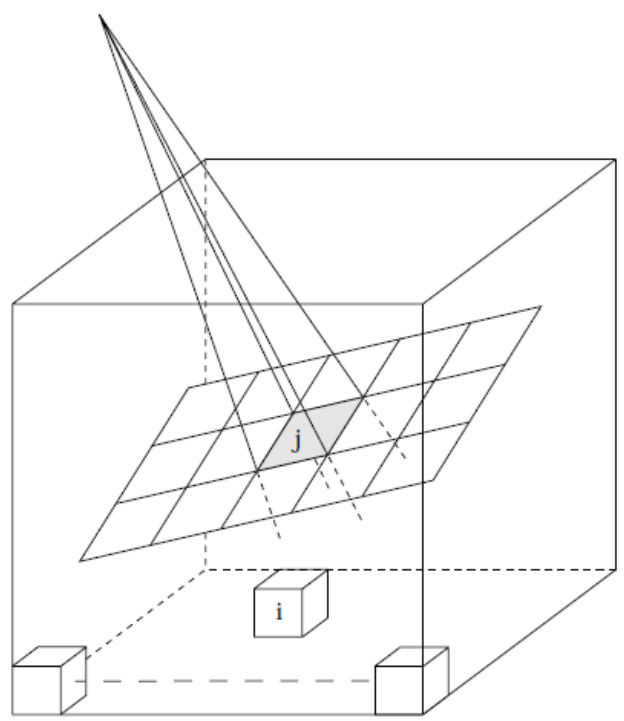

Figure 2.6: Illustration of relationship between bixels and voxels [9].

$$
F_{t}=\sum_{i}^{N_{t}}\left(s_{u}\left[d_{\text {min }}-d_{i}\right]_{+}^{2}+s_{o}\left[d_{i}-d_{\text {max }}\right]_{+}^{2}\right)
$$

where $d_{\min }$ and $d_{\max }$ are the user defined minimum dose and maximum dose to the target, and $s_{u}$ and $s_{o}$ are the user defined penalties for under dose and over dose, and $d_{i}$ is the dose in a voxel as defined by equation $2.2 . N_{t}$ is the number of voxels in the target. The user defines the dose limits and penalties via the KonRad GUI (Figure 2.7). The operator $[.]_{+}$is the positive operator defined as

$$
\begin{aligned}
{[x]_{+} } & =x H(x)=x \quad \text { for } \quad x \geq 0 \\
& =0 \quad \text { everywhere else }
\end{aligned}
$$

For organs at risk (OAR) another objective function is defined: 


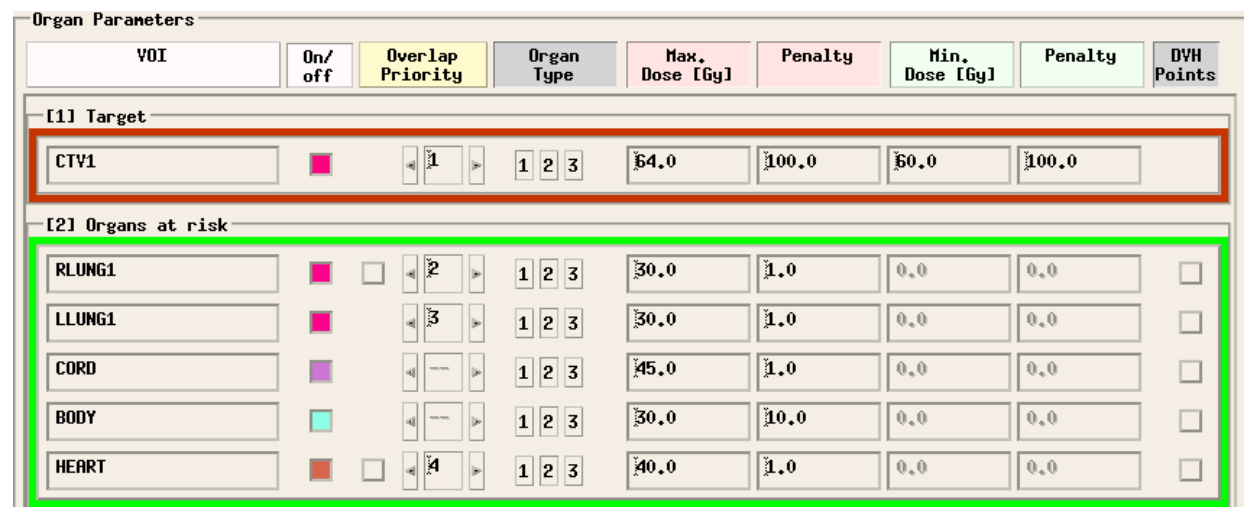

Figure 2.7: Graphical user interface of KonRad treatment plan optimization system allows to choose maximum and minimum dose limits for different organs.

$$
F_{r}=\sum_{i}^{N_{r}} s_{o}\left[d_{i}-d_{\max }\right]_{+}^{2}
$$

where $d_{\max }$ is the maximum dose in the organ, $s_{o}$ is the user defined penalty for over dose and $N_{r}$ is the number of voxels in the organ at risk. The complete objective function is the sum over the number of targets $N_{T}$ and number of organs at risk $N_{R}$.

$$
F=\sum_{i}^{N_{T}} F_{t}(i)+\sum_{i}^{N_{R}} F_{r}(i)
$$

The optimization algorithm: The optimization algorithm is used to search for the optimal weights $w_{j}$ for each bixel for which the objective function value is minimized. The algorithm uses a Newton gradient approach to find the stationary point of the objective function where the gradient is zero. It is an iterative method that assumes that function is quadratic around the minima and uses first and second derivatives to find the stationary points. A generalized form of objective function $F$ given by equation 2.8 and its derivatives can be written as 


$$
\begin{aligned}
& f_{i}=s_{i}\left[d_{i}-d i^{\text {pres }}\right]^{2} \\
& F=\sum_{i} f_{i}
\end{aligned}
$$

$$
\begin{aligned}
\frac{\partial f_{i}}{\partial d_{i}} & =2 s_{i}\left[d_{i}-d_{i}^{p r e s}\right] \\
\frac{\partial^{2} f_{i}}{\partial d_{i}^{2}} & =2 s_{i}
\end{aligned}
$$

By using Newton approach, the weight of each bixel is updated in each iteration by

$$
w_{j}^{t+1}=\left[w_{j}^{t}-\alpha \frac{\frac{d F}{d w_{j}}}{\frac{d^{2} F}{d w_{j}^{2}}}\right]
$$

where $\alpha$ is normalization, or damping, factor. The derivatives of objective function with respect to beam weight $w_{j}$ are

$$
\begin{aligned}
\frac{d F}{d w_{j}} & =\sum_{i} \frac{\partial F}{\partial d_{i}} \frac{\partial d_{i}}{\partial w_{j}} \\
& =\sum_{i} 2 s_{i}\left[d_{i}-d_{i}^{\text {pres }}\right] D_{i j} \\
\frac{d^{2} F}{d w_{j}^{2}} & =\frac{d}{d w_{j}}\left(\frac{d F}{d w_{j}}\right) \\
& =\frac{d}{d w_{j}} \sum_{i} 2 s_{i}\left[d_{i}-d_{i}^{\text {pres }}\right] D_{i j} \\
& =2 \sum_{i} s_{i} D_{i j}^{2}
\end{aligned}
$$

The updated bixel weight in every iterative step becomes

$$
w_{j}^{t+1}=\left[w_{j}^{t}-\alpha \frac{\sum_{i} 2 s_{i}\left[d_{i}-d i^{p} r e s\right] D_{i j}}{2 \sum_{i} s_{i} D_{i j}^{2}}\right]
$$


The optimization starts with all bixel weights equal to one. In each iteration the dose distribution is calculated according to equation 2.2 using the current bixel weight $w_{j}$. Thereafter the value of objective function is calculated. If the relative difference of current and previous objective function is smaller than $0.1 \%$, the optimization stops, otherwise the bixel weight are updated according to equation 2.15 in a new iteration.

\subsection{Treatment planning methods}

The focus of our project is to investigate the methods to manage lung tumor motion for radiotherapy treatment. Therefore we have proposed different methods for generating treatment plans that incorporate tumor motion and its variations. For each of the methods discussed below a treatment plan was optimized. In the following subsections we describe different treatment planning methods and their implementation in detail. Normally a PTV would be required to account for setup and other geometrical errors but no PTV margin was used in any of the planning methods discussed below as our goal was to evaluate the effects of variations in respiratory motion only.

\subsubsection{ITV plan}

ITV based treatment plans were generated for comparison with $4 \mathrm{D}$ optimized plans. The definition of the ITV was given in section 1.4. To delineate the ITV, the full inhale and exhale phase images were fused and a contour was drawn that encompassed the GTVs contoured on both images. The contour was then expanded by $5 \mathrm{~mm}$ to obtain the ITV (Figure 2.8). To create an ITV plan, the ITV was designated as the target in KonRad.

\subsubsection{Nominal 4D plan optimization}

In this section we discuss the implementation of the nominal $4 \mathrm{D}$ optimization approach in KonRad. The plan which accounts for the tumor motion but ignores the variability in motion is called a nominal $4 \mathrm{D}$ plan. Thus the nominal $4 \mathrm{D}$ plan works for a motion that 


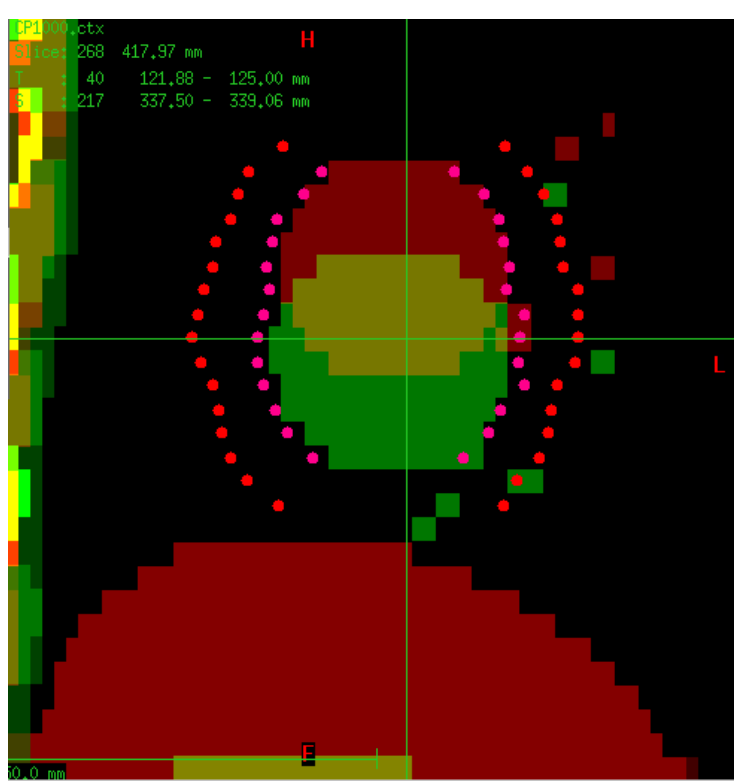

Figure 2.8: A contour (magenta) encompassing GTV of inhale and exhale phases is expanded by $5 \mathrm{~mm}$ to get an ITV (red).

is reproducible. In a nominal plan we optimized the cumulative dose using the exhale phase as a reference phase using the motion pdf approach as described in section 1.10.5. However, KonRad performs only static optimization (i.e 3D optimization) and does not allow modifications to perform 4D optimization. Therefore, we 'tricked' KonRad to perform $4 \mathrm{D}$ optimization by replacing the standard $D_{i j}$ with the 4 DDIJ which is the cumulative dose calculated as follows:

$$
4 D D I J=\sum_{x=0}^{9} D_{i j}(x, 0) p(x)
$$

where $p(x)$ is the weight of phase $x$ and $D_{i j}(x, 0)$ is the dose contribution matrix in phase $x$ mapped to the reference phase. Generating a nominal 4D plan involves the following steps.

(1) Calculation of the dose contribution matrix $D_{i j}(x)$ on each phase: The dose contribution matrix $D_{i j}(x)$ was calculated by optimizing an identical 3D plan in 
each phase. The plans were made identical by copying a plan made on the reference phase to all other phases. The 3D plans on each phase were optimized with the ITV as the target. This was done because KonRad optimizes only preselected bixels based on the target size. However, to account for respiratory motion a larger number of bixels must have non-zero weight.

(2) Deformable image registration: Since the tumor is moving, the linear superposition of doses calculated for preselected bixel area will not give the total dose received by the tumor. The cumulative dose in each voxel of the reference phase must be calculated by determining the corresponding location of that voxel in each phase. This is done by deformable registration of each $4 \mathrm{D}$ CT image to the reference image using a software called ANIMAL (Automated Nonlinear Image Matching and Anatomical Labeling) $[46,47]$. The image registration generates a 3D map of vectors that join voxels in a reference image to the corresponding location in the target image. For each voxel $i$ at location $(i, j, k)$ in the reference phase there is corresponding location $\left(i^{\prime}, j^{\prime}, k^{\prime}\right)$ in another phase $x$ as illustrated in Figure 2.9. These vectors are called deformation vectors as they account the deformation of the voxel in the new location. The images used for registration were the $\mathrm{CT}$ cubes used by KonRad. The quality of each registration was visually checked by evaluating the overlap between the transformed source and target image.

(3) Calculation of 4DDIJ: To calculate the 4DDIJ, dose contribution matrices in the different phases were mapped to the reference phase. To do this, the dose contribution on a given phase $D(x)$ must be mapped to the reference phase to obtain $D(x, 0)$. This requires knowledge of the correspondence between the voxel in the reference phase and the voxel in another phase. The set of vectors calculated in step (2) describes the transformation required to make this correspondence. Using these vectors the dose mapped to a reference voxel at location $(i, j, k)$ is determined by interpolating the dose 


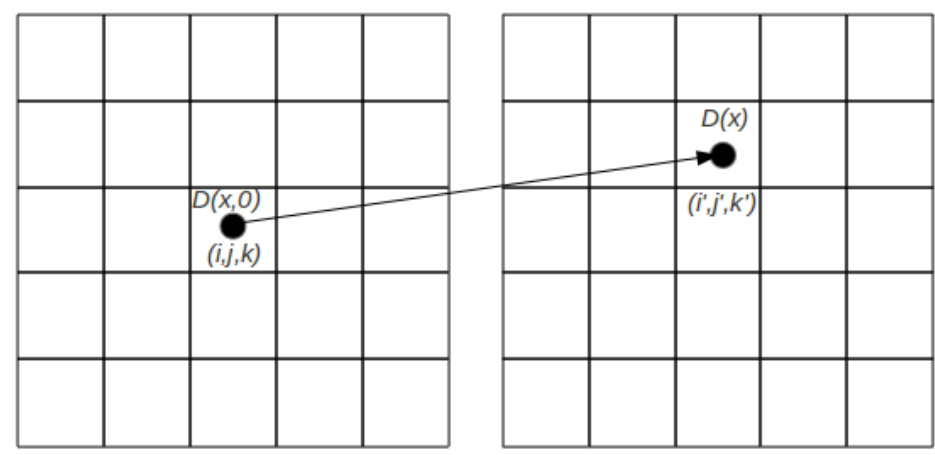

Source image

Target image

Figure 2.9: Illustration of anatomical voxel movement when tumor moves. Arrow indicates deformation vector for voxel $(i, j, k)$. Motion information given by the vector is used to match the corresponding voxels.

at the corresponding location $\left(i^{\prime}, j^{\prime}, k^{\prime}\right)$ on phase $x$. This was done for all voxels each of size $2.62 \times 2.62 \times 2.62 \mathrm{~mm}^{3}$ in a phantom of size $162 \times 103 \times 104 \mathrm{~mm}^{3}$. The sum of the dose mapped to the reference phase from all 9 other phases multiplied by the corresponding phase weight gives 4DDIJ as expressed in Equation 2.16.

(4) 4D optimization The 3DDIJ of the treatment plan in the reference phase was replaced by the 4 DDIJ. The plan optimization was repeated this time with the CTV as a target. The dose distribution obtained from KonRad is now the cumulative dose over the respiratory cycle (equation 2.17).

$$
\left\langle d_{i}\right\rangle=\sum_{x=0}^{9} \sum_{j} D_{i j}(x, 0) p(x) w_{j}
$$

\subsubsection{Robust 4D plan optimization}

The nominal 4D optimization method discussed above does not account for tumor motion variation. If there is any variation in tumor motion the nominal $4 \mathrm{D}$ plan could become suboptimal. In general, tumor motion varies between treatment fractions and also during 
the treatment. Hence there is a need to improve the nominal $4 \mathrm{D}$ plan to address tumor motion variations. A plan that works regardless of the tumor motion variation is called a robust plan. In this section we discuss methods to address the problem of motion variation in IMRT optimization in order to make the plan robust . The two approaches discussed here are the 'average-pdf' and 'worst-case-pdf' approaches.

In the average-pdf approach the 4DDIJ is calculated by using $p_{a v g}(x)$ - the average of the all possible motion pdfs. If there are $n$ individual motion pdfs the average-pdf is given as

$$
p_{\text {avg }}(x)=\frac{p_{1}(x)+p_{2}(x)+\ldots .+p_{n}(x)}{n}
$$

The phase weight $p(x)$ in equation 2.17 was replaced by $p_{a v g}(x)$, to calculate the 4 DDIJ. The 'average-pdf' plan was obtained by optimizing a plan using this 4DDIJ.

$$
\left\langle d_{i}\right\rangle=\sum_{x=0}^{9} \sum_{j} D_{i j}(x, 0) p_{a v g}(x) w_{j}
$$

An alternative to this approach is the worst-case pdf approach. In this approach we first optimized two nominal 4D plans, each on a different pdf representing a 'worst-case' motion variation. These plans were then combined by calculating the average of the bixel weights (equation 2.20) for each beam. Figure 2.10 shows bixel weight maps of two 'worst-case' nominal plans and their average.

$$
w_{j}(a v g)=\frac{w_{j}(1)+w_{j}(2)}{2}
$$

where $w_{j}(1)$ and $w_{j}(2)$ are the bixel weights of the worst case plans. To calculate the cumulative dose distribution from the 'worst-case' $4 \mathrm{D}$ plan, the bixel weight $w_{j}$ in equation 2.17 is replaced by the average bixel weight, $w_{j}(a v g)$ and combined with the 4DDIJ according to the following formula: 

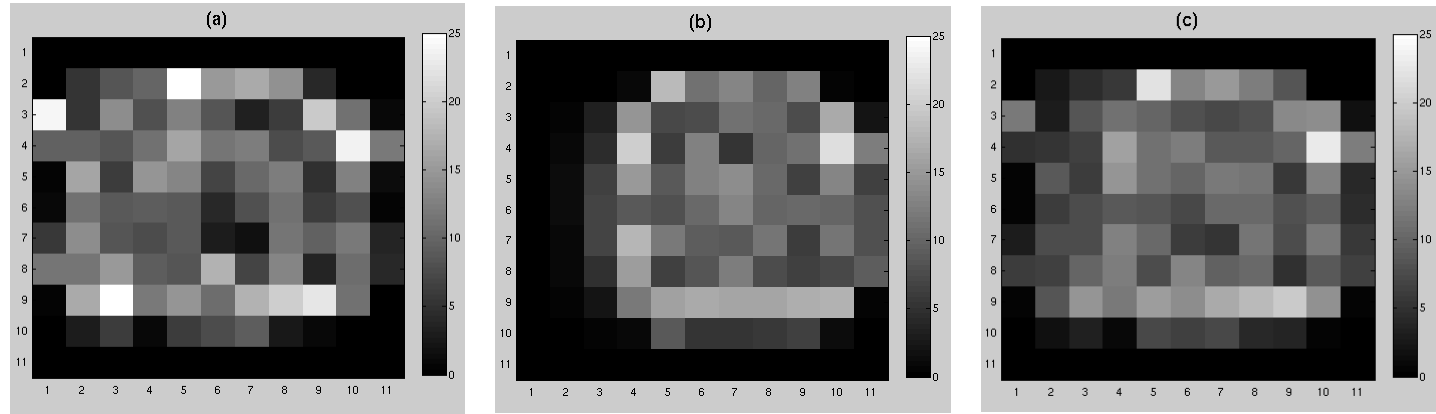

Figure 2.10: Bixel intensity maps in 'worst-case' pdf approach. Figure (a) and (b) are bixel intensity maps of 4D nominal plans optimized for two worst case plans. Figure (c) is the average of (a) and (b).

$$
\left\langle d_{i}\right\rangle=\sum_{x=0}^{9} \sum_{j} D_{i j}(x, 0) p(x) w_{j}(\text { avg })
$$

\subsection{Validation of $4 \mathrm{D}$ optimization}

The following tests were performed to validate the nominal $4 \mathrm{D}$ optimization method, in particular the calculation of the 4DDIJ. We tested three scenarios: ITV plan applied to 4DDIJ, CTV plan applied to 4DDIJ and a 'tracking plan' applied to a 4DDIJ.

ITV plan applied to 4DDIJ: The dose distribution was obtained by applying the ITV plan to a 4DDIJ. Since the ITV encompasses the CTVs in all phases, it is expected that the dose distribution should cover the CTV, if our 4DDIJ calculation is correct.

CTV plan applied to 4DDIJ: A treatment plan which delivers 60 Gy to the CTV at exhale was made. We applied this plan to the 4DDIJ. Since this plan only covers CTV at exhale, we expect that the delivered dose should only partly cover the CTV.

Tracking plan: For this test, a treatment plan was optimized on the CTV delineated on the inhale CT image. A 4DDIJ was calculated using only the inhale dose contribution. 
When the inhale plan is applied to this 4DDIJ we expect that the dose distribution should perfectly cover the CTV at exhale.

\subsection{Motion variation scenarios}

Tumor motion information was obtained from 4D CT images generated by XCAT. Tumor motion in a respiration cycle of 5 sec was divided into ten different phases. Since XCAT generates these phases with exhale as the reference phase, we measured the displacement of each phases from the exhale phase. The phase vs displacement in the superior inferior direction is given in Table 2.11 and Figure 2.11.

Table 2.1: Tumor displacement

\begin{tabular}{ccccccccccc}
\hline Phase & 1 & 2 & 3 & 4 & 5 & 6 & 7 & 8 & 9 & 10 \\
\hline Displacement $(\mathrm{mm})$ & 0 & 3.32 & 8.2 & 15.23 & 17.58 & 16.01 & 12.69 & 8.2 & 5.27 & 0.81 \\
\hline
\end{tabular}

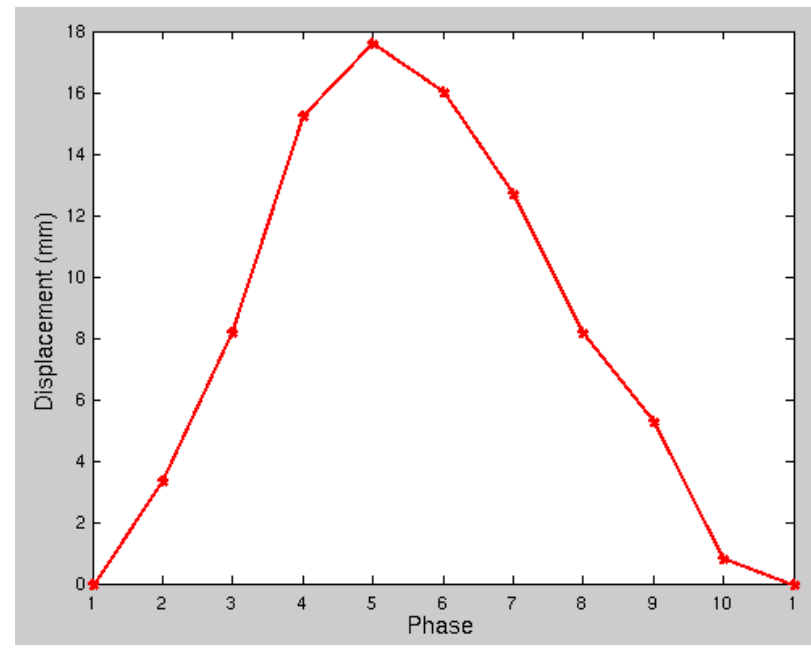

Figure 2.11: Tumor displacement in a respiration cycle of 5 sec as generated by XCAT.

To test the robustness of the proposed 4D optimization methods different scenarios of tumor motion variation were generated. All the scenarios discussed below were created manually, however they are close to reality. We considered the following two different 
scenarios.

Scenario 1 - more time inhaling vs exhaling: This scenario considers variation in the time spent inhaling versus time spent exhaling. If a patient spends more time inhaling, the tumor will also take more time to reach the maximum displacement than to return from the maximum displacement to the exhale phase. Similarly, if the patient takes more time exhaling, the tumor will also take more time to return from maximum displacement to the exhale phase. Four different pdfs that model these scenarios are in Table 2.2.

Table 2.2: Tumor motion variation scenario: more time inhaling vs exhaling

\begin{tabular}{cccccc}
\hline \hline phase & case3 & case14 & case17 & case20 & average-pdf \\
& $p(x)$ & $p(x)$ & $p(x)$ & $p(x)$ & $p_{\text {avg }}$ \\
\hline 1 & 0.243 & 0.08 & 0.25 & 0.14 & 0.17852 \\
2 & 0.17 & 0.06 & 0.2 & 0.093 & 0.13075 \\
3 & 0.074 & 0.05 & 0.15 & 0.074 & 0.087 \\
4 & 0.057 & 0.04 & 0.1 & 0.056 & 0.06325 \\
5 & 0.056 & 0.03 & 0.04 & 0.037 & 0.04075 \\
6 & 0.037 & 0.04 & 0.03 & 0.056 & 0.04075 \\
7 & 0.056 & 0.1 & 0.04 & 0.057 & 0.06325 \\
8 & 0.074 & 0.15 & 0.05 & 0.074 & 0.087 \\
9 & 0.093 & 0.2 & 0.06 & 0.17 & 0.13075 \\
10 & 0.14 & 0.25 & 0.08 & 0.243 & 0.17825 \\
\hline Total probability & 1 & 1 & 1 & 1 & 1 \\
\hline
\end{tabular}

Figure 2.12 illustrates the location of each phase in the respiration cycle. Phase 1 to phase 5 are inhaling phases, whereas phase 6 to phase 10 are exhaling phases. Phase 5 corresponds to full inhale as it is at the maximum displacement. These phases are equally spaced in time. However due to asymmetry of time inhaling vs exhaling there are more phases on exhaling phases. The inhaling time is proportional to the sum of weights of phase 1 to phase 5, and exhaling time is proportional to sum of weights of phase 6 to phase 10. In 'case3' and 'case17' the relative inhaling time is $60 \%$ and $74 \%$ respectively, and therefore they are more time inhaling cases. Similarly, in 'case14' and 


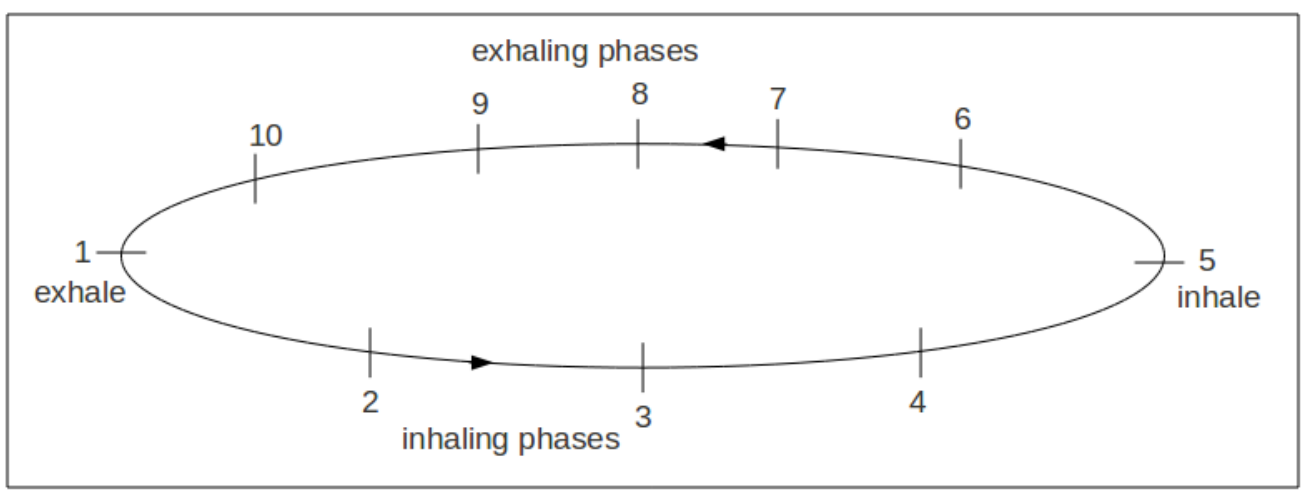

Figure 2.12: Illustration of phases in a respiration cycle. The numbers indicate the different phases of tumor motion, and the ellipsoid represents the periodic nature of the motion but not the trajectory.

'case20' the relative exhaling time is $74 \%$ and $60 \%$ respectively, and therefore they are more time exhaling cases. The average-pdf, $p_{a v g}$ of all these cases is shown in Table 2.2.

Scenario 2 - amplitude variation: Another scenario of tumor motion variation is the variation in the maximum tumor displacement. The variation in tumor displacement scenarios are presented in table 2.3 .

Table 2.3: Tumor motion variation scenario: amplitude variation

\begin{tabular}{cccccccc}
\hline \hline phase & $\begin{array}{c}\text { displacement } \\
\mathrm{x}(\mathrm{mm})\end{array}$ & $\begin{array}{c}\text { case22 } \\
p(x)\end{array}$ & $\begin{array}{c}\text { case23 } \\
p(x)\end{array}$ & $\begin{array}{c}\text { case24 } \\
p(x)\end{array}$ & $\begin{array}{c}\text { case25 } \\
p(x)\end{array}$ & $\begin{array}{c}\text { case26 } \\
p(x)\end{array}$ & $\begin{array}{c}\text { average } \\
\text { pdf }\left(p_{\text {avg }}\right)\end{array}$ \\
\hline 1 & 0 & 0.145 & 0.2 & 0.112 & 0.134 & 0.124 & 0.144 \\
2 & 3.32 & 0.145 & 0.2 & 0.111 & 0.134 & 0.136 & 0.1476 \\
3 & 8.2 & 0.14 & 0.2 & 0.111 & 0.132 & 0.14 & 0.1384 \\
4 & 15.23 & 0 & 0 & 0.111 & 0.12 & 0 & 0.034 \\
5 & 17.58 & 0 & 0 & 0 & 0 & 0 & 0 \\
6 & 16.01 & 0 & 0 & 0.111 & 0.12 & 0 & 0.032 \\
7 & 12.69 & 0.14 & 0 & 0.111 & 0.12 & 0.15 & 0.106 \\
8 & 8.2 & 0.14 & 0 & 0.111 & 0.12 & 0.15 & 0.106 \\
9 & 5.27 & 0.145 & 0.2 & 0.111 & 0.12 & 0.15 & 0.146 \\
10 & 0.81 & 0.145 & 0.2 & 0.111 & 0.12 & 0.15 & 0.146 \\
\hline Total & & 1 & 1 & 1 & 1 & 1 & 1 \\
probability & & & & & & & \\
\hline
\end{tabular}


We generated scenarios with different amplitudes by putting the weight equal to 0 for certain phases. For example, in full inhalation, the tumor amplitude is $17.58 \mathrm{~mm}$, but in case 25 we make the amplitude to be only $16.01 \mathrm{~mm}$ by putting weight equal to 0 for phase 5. Similarly, in case22 the amplitude is made to be $12.69 \mathrm{~mm}$ by putting phase weight equal to 0 in phase 4,5 and 6 . Putting the phase weight equal to 0 means that tumor does not spend any time in that phase. The motion amplitude is measured with respect to the position in reference phase. We have not considered the case of baseline shift.

\subsection{Testing sensitivity of plans to motion variations}

We expect that the nominal $4 \mathrm{D}$ plans are sensitive to any variation in motion under the scenarios discussed above. Similarly we also expect that the robust $4 \mathrm{D}$ plans are invariant under similar variation in tumor motion. To compare with conventional planning techniques we also perform the same sensitivity tests on the ITV plan to demonstrate any advantages of robust 4D plans over ITV-based plans in terms of lung dose sparing.

The sensitivity test of a given plan is done by projecting its bixel weights over the 4DDIJ calculated using different motion pdfs. For example, a nominal 4D plan is optimized for a given $p(x)$, say case22. To check if it is sensitive to other type of motion, we apply the bixel weights $w_{j}$ of this plan to 4DDIJ in equation 2.16 calculated using different $p(x)$, say either case 24,25 or 26 .

Similarly, to check the sensitivity of a robust plan we apply the bixel weights $w_{j}$ of a robust plan to the 4 DDIJ in equation 2.16 calculated using different $p(x)$. We expect that the robust plan is insensitive to any variation in motion. For comparison, a similar test is done for the ITV plan by projecting its bixel weights to different 4DDIJs. This comparison enables us to evaluate if the robust plans are advantageous over the ITV plan under similar motion variations. For a quantitative comparison we use parameters 
such as the volume of the CTV that receives at least $95 \%$ of the prescribed dose (V95), the lung volume that receives at least 20 Gy (V20), the minimum, mean and maximum dose in the CTV, and the maximum dose in the spinal cord, heart and body.

\subsection{Dose prescription and OAR dose tolerance}

All the treatment plans used in this study were optimized for a prescribed dose of 60 Gy. To evaluate the plan we used the ICRU guidelines [16] that states that the dose in the target volume should be at least $95 \%$ of the prescribed dose and not exceed $107 \%$ of the prescribed dose. This means that the CTV in our plans should receive at least 57 Gy but not more than 64.2 Gy. The dose tolerance limits for organs at risk (OAR) were taken from RTOG protocol 0117 [48] for IMRT treatment of lung cancer. In this protocol the maximum heart dose is $40 \mathrm{~Gy}$, maximum spinal cord dose 45 Gy and the lungs must have V20 $\leq 30 \%$. 


\section{Chapter 3}

\section{Results and discussion}

\subsection{Validation test results}

\subsubsection{ITV plan applied to 4DDIJ}

Figure 3.1 shows the dose distribution around the CTV when the ITV plan is applied to the exhale phase. The CTV dose meets the ICRU criteria. The CTV minimum dose is greater than $95 \%$ of prescribed dose (57 Gy), and less than $107 \%$ of the prescribed dose (64 Gy). Since the ITV covers the CTV in all phases we expect that the CTV is covered by the accumulated dose from the ITV plan. Figure 3.2 shows the accumulated dose distribution where the $95 \%$ isodose curve fully covers the CTV as expected.

\subsubsection{CTV plan applied to 4DDIJ}

The CTV plan has a smaller beam size compared to the ITV plan. Figure 3.3 shows the dose distribution on the exhale phase for a plan where the exhale CTV was the target volume. Since the exhale CTV does not encompass the CTVs of the other phases, the cumulative dose from such a plan results in the tumor being highly under dosed as shown in Figure 3.4. 


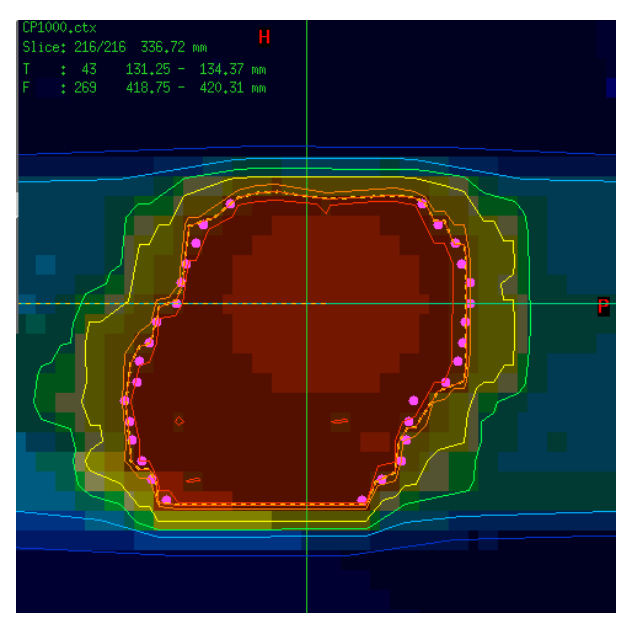

Figure 3.1: ITV plan applied to Exhale phase. Dashed yellow line indicate 95\%(57 Gy) isodose line. Pinl dots represent the ITV.

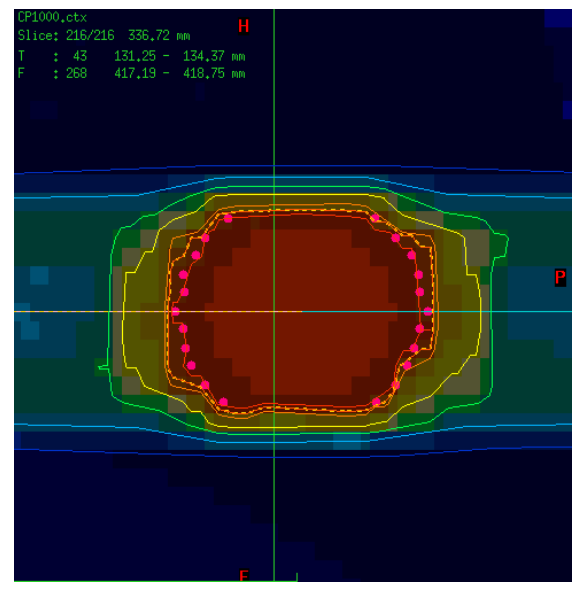

Figure 3.3: Dose distribution of CTV plan on Exhale phase. Dashed yellow line indicate 95\%(57 Gy) isodose line. Red dots represent exhale CTV.

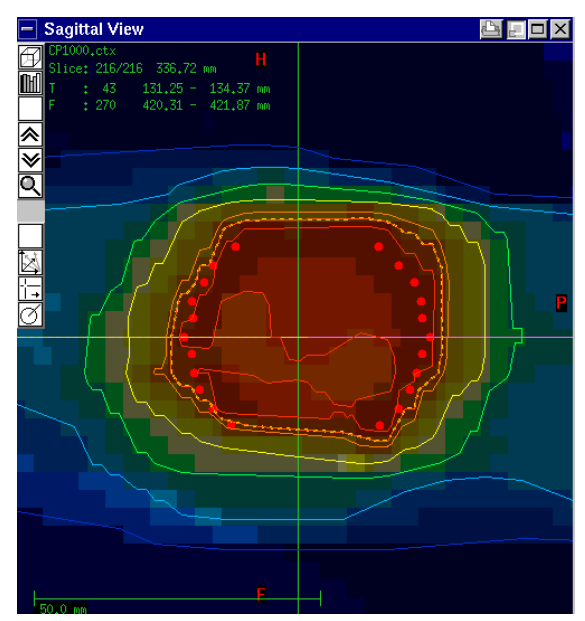

Figure 3.2: Accumulated dose over 10 phases from ITV plan. Dashed yellow line indicate 95\%(57 Gy) isodose line. Red dots represent the exhale CTV.

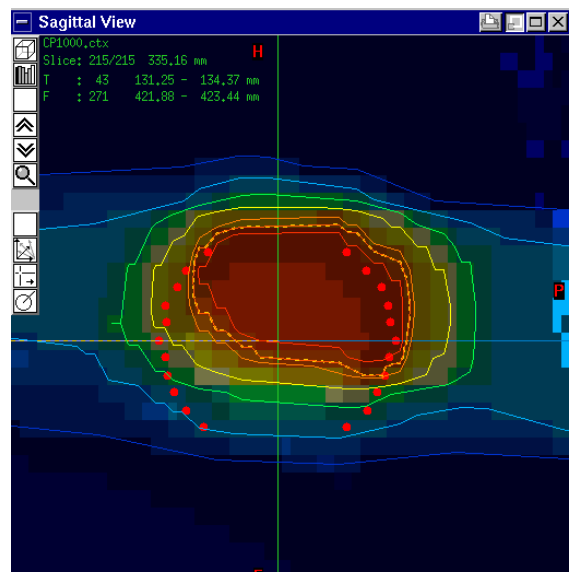

Figure 3.4: Accumulated dose distribution for CTV plan. Dashed yellow line indicate 95\%(57 Gy) isodose line. Red dots represent exhale CTV. 


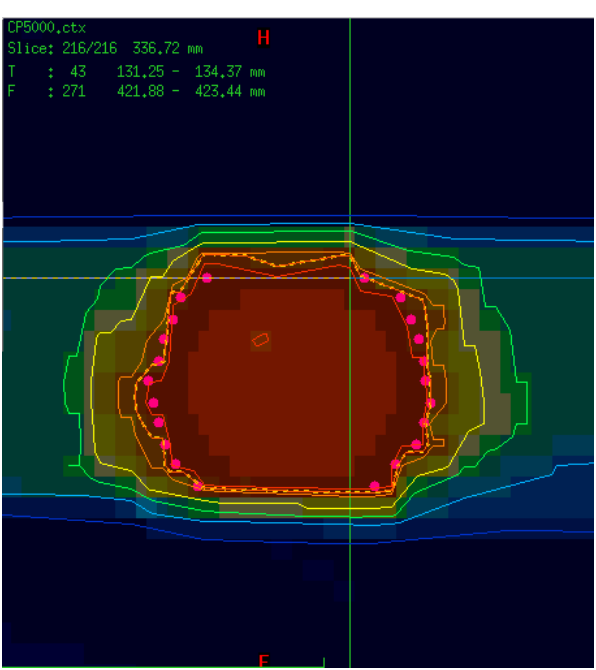

Figure 3.5: Dose distribution of tracking plan on Inhale phase. Red dots represent the CTV in inhale.

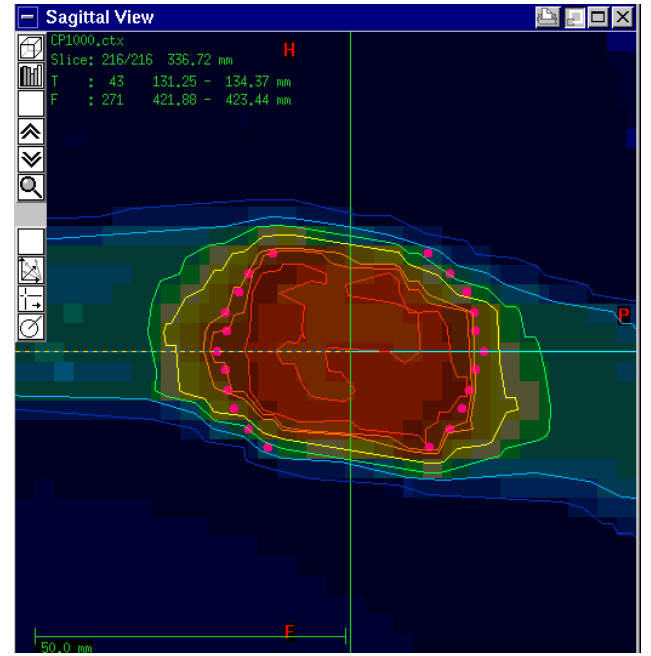

Figure 3.6: Accumulated dose distribution of tracking plan. Red dots represent the CTV in exhale.

\subsubsection{Tracking plan}

This is another method of checking the 4DDIJ calculation code. A plan was made using the CTV at maximum displacement (full inhale) as the target volume. Then the dose was mapped to the position of zero displacement (full exhale). In this case the accumulated dose (Equation 2.17) was calculated only from the inhale phase. In other words this method is similar to tumor tracking. If the image registration and dose accumulation are correctly performed, then it is expected that the accumulated dose distribution should conform perfectly to the tumor at exhale.

Figure 3.5 shows the dose distribution from this tracking plan in the inhale phase. Figure 3.6 shows the accumulated dose on the exhale phase. In Figure 3.6 we see that dose is mapped inside the exhale CTV however it is not perfectly covered. The reason for this could be imperfect image registration. While comparing the registered exhale and inhale images, we found that the alignment of the tumor in both images was not perfect along the tumor borders (Figure 3.7). The transformed exhale tumor is larger than the inhale tumor. So when dose is mapped from inhale to exhale it maps to a 

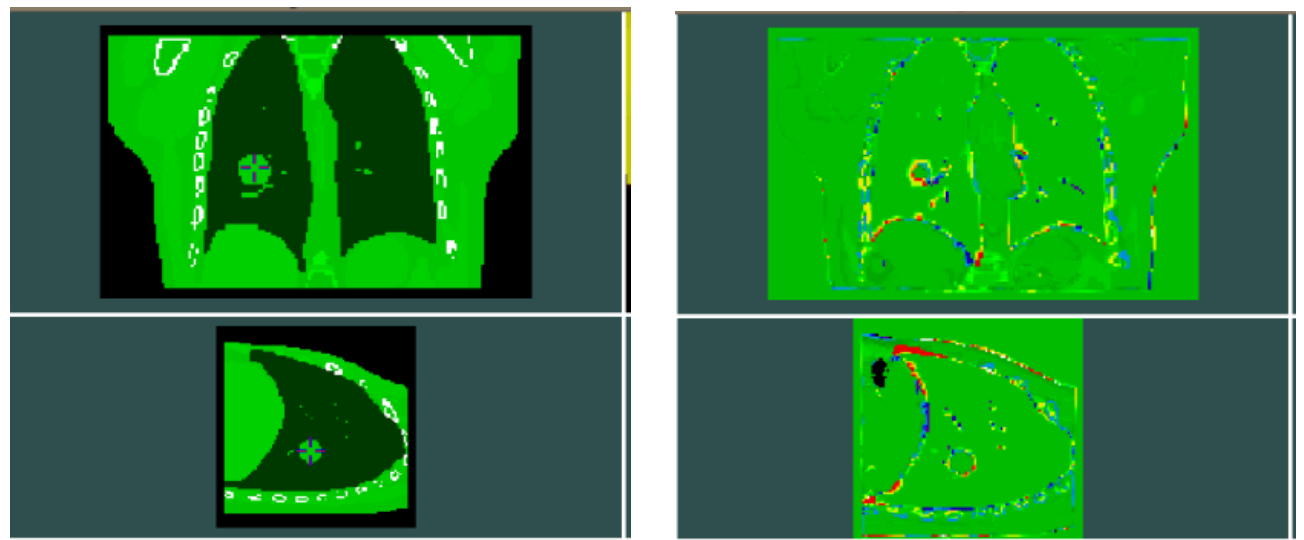

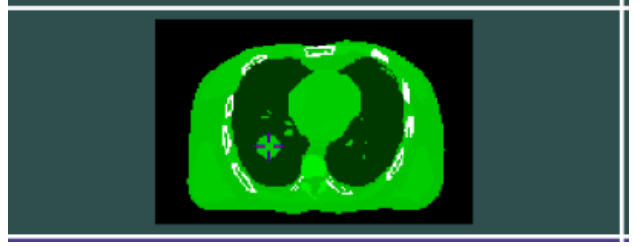

(a)

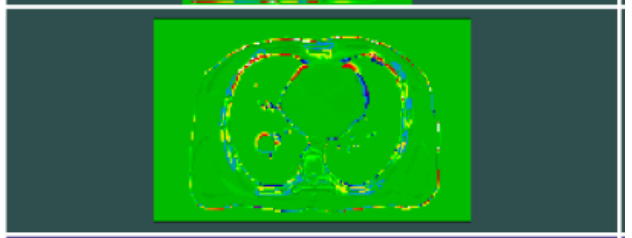

(b)

Figure 3.7: Figures showing the imperfect image registration. (a) Inhale image and (b) inhale image subtracted from transformed exhale to inhale phase. Yellow and red color represent a positive image difference.

smaller volume, which causes dose to not cover CTV at exhale.

\subsection{Motion variation scenario 1: more time inhaling vs exhaling}

The tumor motion is guided by the patient breathing pattern. Thus, tumor motion will change according to the time the patient takes to inhale and exhale. We considered four different cases of patient breathing style by varying the time for inhaling and exhaling. They are case3, case14, case17 and case20. Figure 3.8 illustrates the tumor motion variation scenario when patient takes different amounts of time inhaling vs exhaling. In case 17 patient spends $74 \%$ of the breathing cycle in inhaling and $26 \%$ exhaling. Case14 is just opposite to case17. Similarly, in case 3 the patient spends $60 \%$ of the breathing 
cycle inhaling and $40 \%$ exhaling. The case 20 is opposite to case 3.

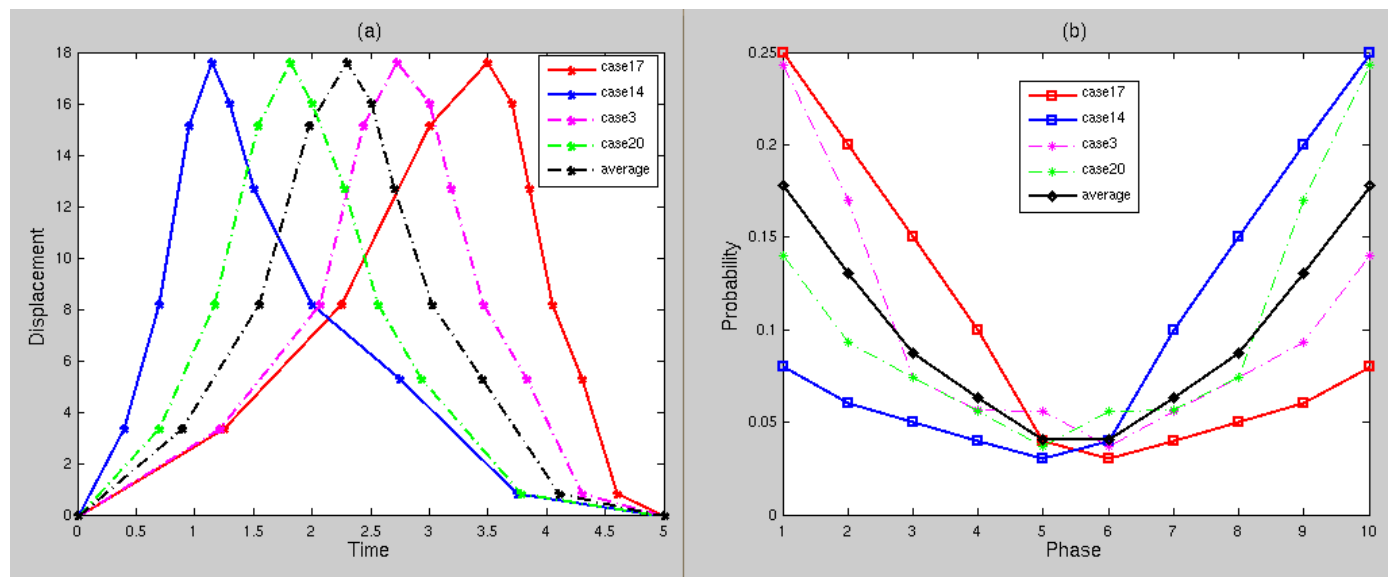

Figure 3.8: (a) The tumor displacement as a function of time and (b) phase weight as a function of phase.

\subsubsection{Nominal 4D plan}

As discussed in Chapter 2, nominal 4D plans account for the tumor motion but do not account for the variations in the tumor motion. We made a nominal $4 \mathrm{D}$ plan using the case17 pdf and applied this plan to the other cases to see if the plan is sensitive to the motion variations. The solid line in Figure 3.9 shows the dose volume histograms of nominal plan using case 17. The dose statistics for different organs are shown in Table 3.1. The vertical dashed lines in Figure 3.9 indicate the lower and upper CTV dose criteria. The DVH and dose statistics for the nominal plan on case17 all meet the plan criteria, except for CTV max dose which is slightly higher.

The dashed lines in Figure 3.9 show the dose volume histograms when the nominal $4 \mathrm{D}$ plan is applied to the three different cases. Table 3.1 gives the corresponding dose statistics. These DVHs show that the nominal $4 \mathrm{D}$ plan is sensitive to motion variation to some extent as the DVHs deviate from nominal plan DVH. The nominal $4 \mathrm{D}$ plan is most sensitive to case 14 in which time spent inhaling is $26 \%$ and time spent exhaling is 


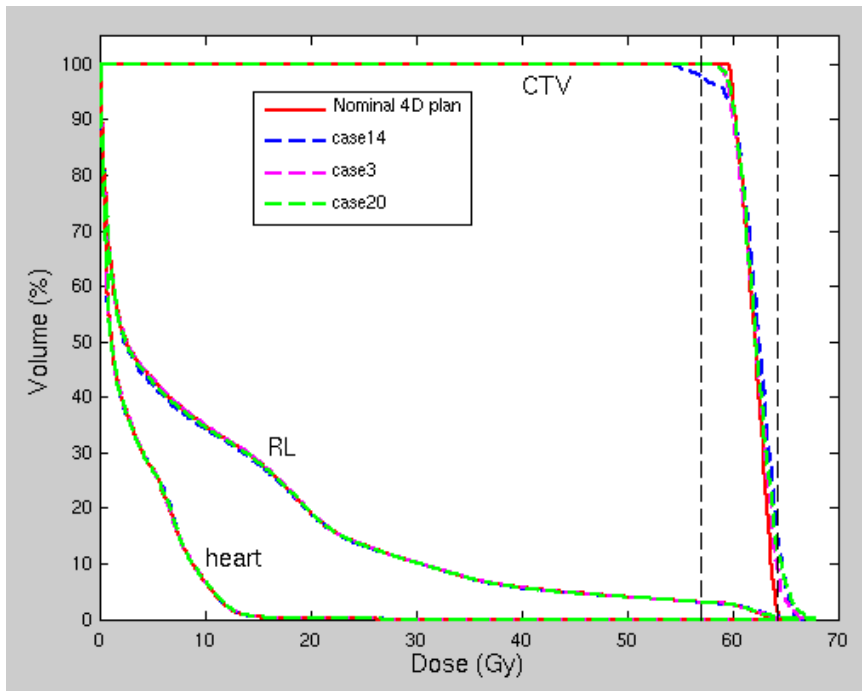

Figure 3.9: Dose volume histograms (DVH) showing the sensitivity of a nominal 4D plan to motion variation scenario 1 . The vertical dashed lines represent ICRU min and max dose criteria.

Table 3.1: Dose statistics for Nominal 4D plan applied to the motion variations in scenario 1. $(\mathrm{RL}=$ right lung, $\mathrm{LL}=$ left lung, $\min / \mathrm{mean} / \mathrm{max}=\operatorname{minimum} / \mathrm{mean} / \mathrm{maximum}$ dose in Gy).

\begin{tabular}{|c|c|c|c|c|}
\hline & $\begin{array}{c}\text { nominal } \\
\text { 4D plan }\end{array}$ & case14 & case3 & case20 \\
\hline RL(max) & 62.6 & 63.5 & 62.7 & 62.9 \\
\hline LL(max) & 14.5 & 14.5 & 14.6 & 14.6 \\
\hline Cord(max) & 16.6 & 16.5 & 16.6 & 16.5 \\
\hline Body(max) & 43.2 & 43.1 & 43.0 & 43.0 \\
\hline Heart(max) & 26.3 & 26.8 & 26.6 & 27.0 \\
\hline CTV(min) & 59.2 & 53.3 & 58.1 & 57.5 \\
\hline CTV(mean) & 62.0 & 62.1 & 62.1 & 62.2 \\
\hline CTV(max) & 64.6 & 66.7 & 66.8 & 67.8 \\
\hline V20(\%-lung) & 17.4 & 17 & 17.4 & 17.1 \\
\hline V95(\%) & 100 & 97.6 & 97.8 & 100 \\
\hline
\end{tabular}

74\%. For this case the minimum dose to the CTV is below the ICRU criteria of 57 Gy. The maximum CTV dose for all test cases is higher than the ICRU criteria of 64 Gy. 


\subsubsection{Robust plan using average pdf}

Designing a robust plan using the average-pdf approach was discussed in Chapter 2. In the average pdf approach we used the average of the pdfs of the cases discussed in scenario 1 to make a robust plan. The robust plan was then applied to each of the cases separately to check the sensitivity of the robust plan to motion variations. The solid line in Figure 3.10 shows the DVH for the robust plan optimized with the average pdf.

The dashed lines in Figure 3.10 shows the dose volume histograms for the average pdf plan applied to the different cases. We can see that the CTV is covered by $95 \%$ of the prescribed dose in each of the cases except in case14 which looks slightly under dosed in the DVH plot. However, if we analyze the cumulative dose distribution shown in Figure 3.11, the $95 \%$ isodose appears to almost cover the CTV fully. Therefore only a few voxels of the CTV are receiving less than 57 Gy. Compared to the sensitivity of nominal 4D plan under the same motion variation, in the average pdf plan the CTV minimum dose is improved for each case. The maximum CTV dose is also reduced except for case3 where it is increased by 0.3 Gy (Table 3.2). The heart and body max dose are increased, but the increase is small. The OAR max doses are below the limits in our plan criteria described in Chapter 2 section 2.6.

Table 3.2: Dose statistics for average-pdf plan applied to cases in motion variation scenario 1.(RL=right lung, $\mathrm{LL}=$ left lung, $\mathrm{min} / \mathrm{mean} / \mathrm{max}=\mathrm{minimum} / \mathrm{mean} / \mathrm{maximum}$ dose in Gy).

\begin{tabular}{|c|c|c|c|c|c|}
\hline & $\begin{array}{c}\text { average } \\
\text { pdf plan }\end{array}$ & case17 & case14 & case3 & case20 \\
\hline RL(max) & 62.7 & 62.5 & 63.4 & 62.6 & 62.8 \\
\hline LL(max) & 14.6 & 14.6 & 14.5 & 14.8 & 14.9 \\
\hline Cord (max) & 18.3 & 18.3 & 17.9 & 18.2 & 18.3 \\
\hline Body(max) & 44.4 & 44.5 & 44.5 & 44.3 & 44.4 \\
\hline Heart(max) & 27.3 & 26.9 & 27.6 & 27.2 & 27.5 \\
\hline CTV(min) & 59.0 & 58.0 & 54.6 & 58.4 & 58.8 \\
\hline CTV(max) & 64.6 & 64.8 & 66.2 & 67.1 & 66.4 \\
\hline V20(\%-lung) & 17.1 & 17.0 & 17.3 & 17.2 & 17.5 \\
\hline V95(\%) & 100 & 100 & 97.8 & 100 & 100 \\
\hline
\end{tabular}




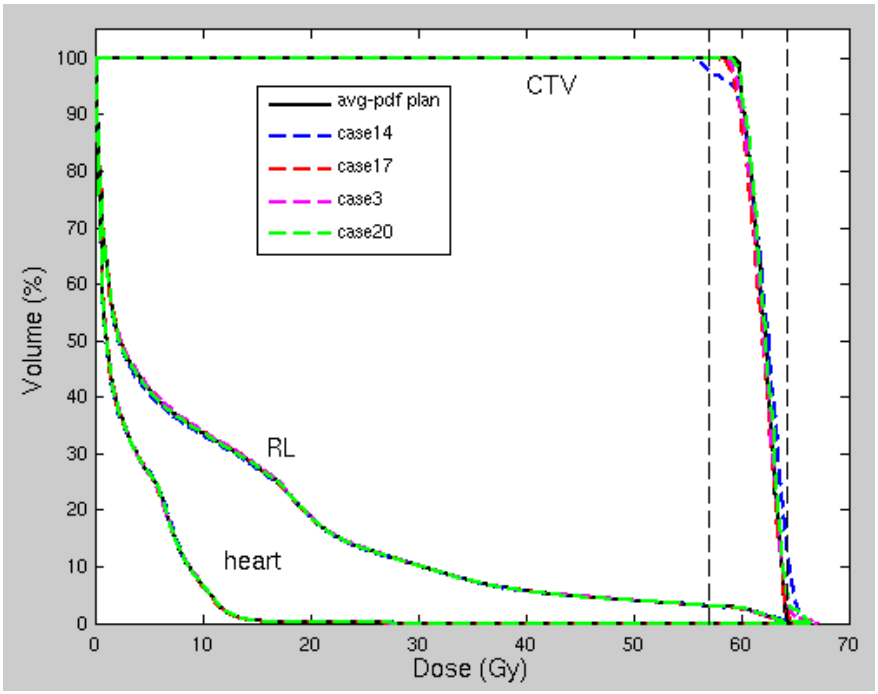

Figure 3.10: Dose volume histograms showing the sensitivity of average pdf plan under motion variation scenario 1 . Vertical dashed lines indicate ICRU $\min / \max$ dose criteria.

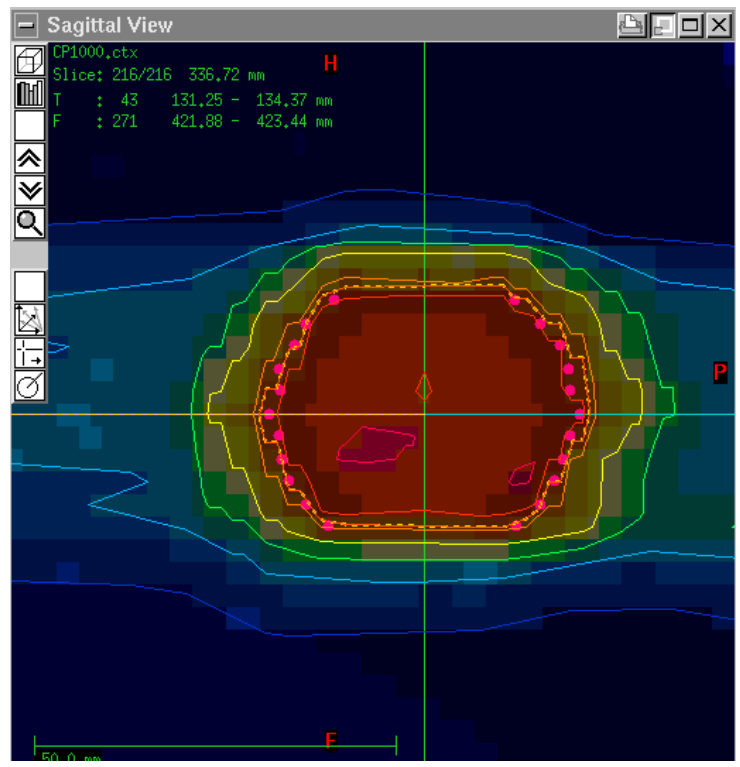

Figure 3.11: Dose distribution when average pdf plan is applied to case14. Yellow dashed line indicates $95 \%$ of prescribed dose. Red dots represent CTV at exhale. 
The reason for the average pdf plan working for all cases but case14 can be found if we look the pdf table in Chapter 2 section 2.2 and compare the phase weights. We can see that the average pdf has similar phase weights as in case 3 and case20 where the inhale phases (phase 4, 5,6 and 7) are weighted less. But in case14 phases 7 and 8 are weighted more than in the average pdf plan. It is also interesting to note that in case17 phases 3 and 4 are weighted more than in the average pdf plan. However, the CTV is fully covered in case17 but not in case14. Further study is required to fully understand why the CTV is under dosed in case14 but not in case17. The reason for nominal plan to be more sensitive than average pdf plan can also be explained using the same pdf table. The nominal plan was made in case 17 which is heavily weighted only in inhaling phases where as average pdf puts weights more on exhale phases.

\subsubsection{Robust plan using worst case pdfs}

A robust $4 \mathrm{D}$ treatment plan designed using the worst-case pdf approach was also tested. In scenario 1, the worst case pdfs were identified as being case14 where the inhaling/exhaling time is $26 \% / 74 \%$ and case 17 where the inhaling/exhaling time is $74 \% / 26 \%$. These cases were considered as the worst cases because they represent the two extremes of the phase weighting variations. The beam intensity maps of the nominal $4 \mathrm{D}$ plans made for these two pdfs were combined with equal weight in each case and then applied to each of the four test cases separately.

The CTV dose volume histograms and dose statistics for the worst case pdf plan applied to all the test cases are presented in Figure 3.12 and Table 3.3. In all the cases the CTV is covered by $95 \%$ (57 Gy) of the prescribed dose (60 Gy). It can be seen that the worst-case pdf approach results in a more robust plan than the average-pdf approach. Furthermore the maximum CTV dose is reduced compared to the avg pdf plans. 


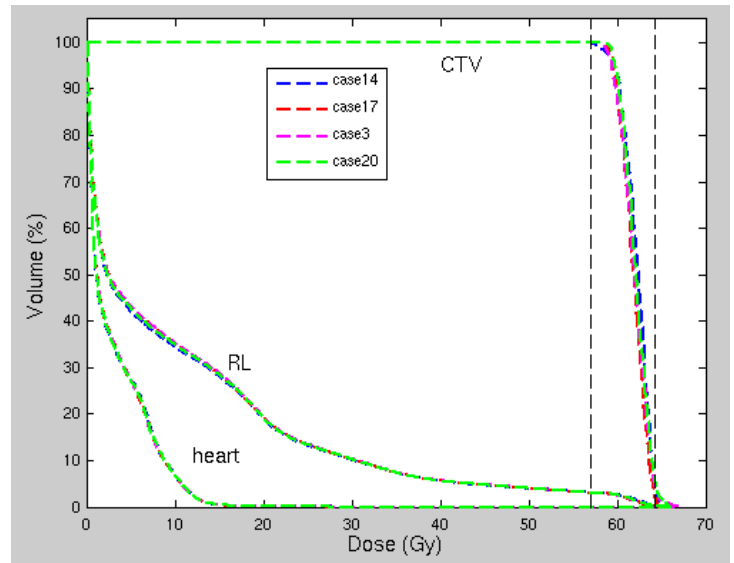

Figure 3.12: Dose volume histogram of robust plan using worst case approach in motion variation scenario 1 . Vertical dashed lines represent ICRU $\min / \max$ dose criteria.

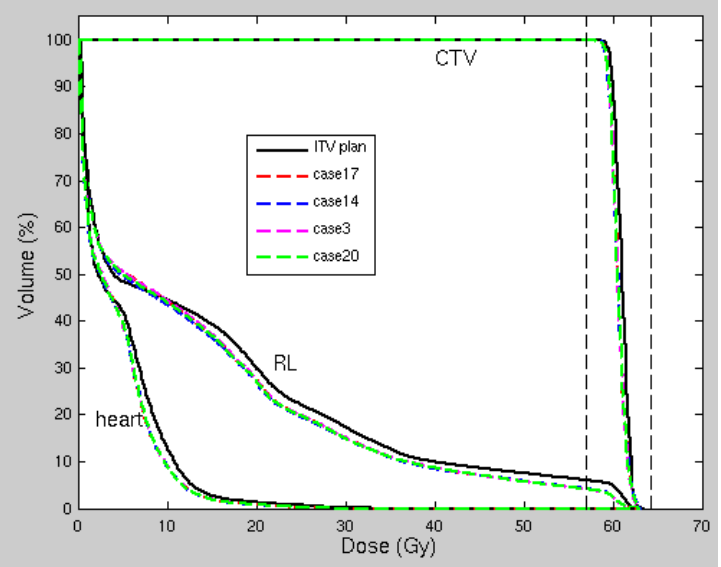

Figure 3.13: Dose volume histograms of ITV plan under motion variation scenario 1 . Vertical dashed lines represent ICRU $\min / \max$ dose criteria.

Table 3.3: Dose statistics for worst case pdf plan applied to motion variation scenario 1. $(\mathrm{RL}=$ right lung, $\mathrm{LL}=$ left lung, $\mathrm{min} / \mathrm{mean} / \mathrm{max}=\operatorname{minimum} / \mathrm{mean} /$ maximum dose in Gy)

\begin{tabular}{|c|c|c|c|c|}
\hline & case14 & case17 & case3 & case20 \\
\hline RL(max) & 63.0 & 62.2 & 63.9 & 62.4 \\
\hline LL(max) & 14.4 & 14.5 & 14.7 & 14.7 \\
\hline Cord(max) & 17.6 & 17.7 & 17.7 & 17.6 \\
\hline Body(max) & 43.6 & 43.6 & 43.5 & 43.5 \\
\hline Heart(max) & 27.6 & 27.0 & 27.6 & 27.4 \\
\hline CTV(min) & 56.5 & 58.3 & 58.0 & 58.5 \\
\hline CTV(max) & 65.4 & 64.7 & 66.8 & 66 \\
\hline V20(\%-lung) & 17.4 & 17.4 & 17.7 & 16.9 \\
\hline V95(\%) & 99.8 & 100 & 100 & 100 \\
\hline
\end{tabular}

\subsubsection{ITV plan:}

We compared the robust plans discussed above with an ITV plan under the same motion variations to see if the robust plans are advantageous over the ITV plan. The ITV encompasses the CTVs of all 10 phases. Therefore, we expect that changing the weighting of each phase will not have any impact on the accumulated dose from an ITV plan.

It can be seen in Figure 3.13 that the ITV plan is not sensitive to motion variations 
as expected. However, Table 3.4, shows that V20 for right lung is larger in the ITV plan compared to any of the $4 \mathrm{D}$ plans. While this value of V20 is still acceptable, it limits the potential for escalating the dose delivered to the tumor. The difference between the ITV plan and robust plans can also be seen if we compare dose distribution of the ITV plan and robust plan applied to the same motion variation case, say case17. Figure 3.14 shows that the ITV plan puts extra dose to the healthy tissue outside CTV, whereas the average pdf plan applied to the same case (Figure 3.15 ) is very conformal to the CTV. The V20 tells us that about $10 \%$ more of the lung volume is receiving $\geq 20$ Gy. The heart max dose is also increased in the ITV plan.

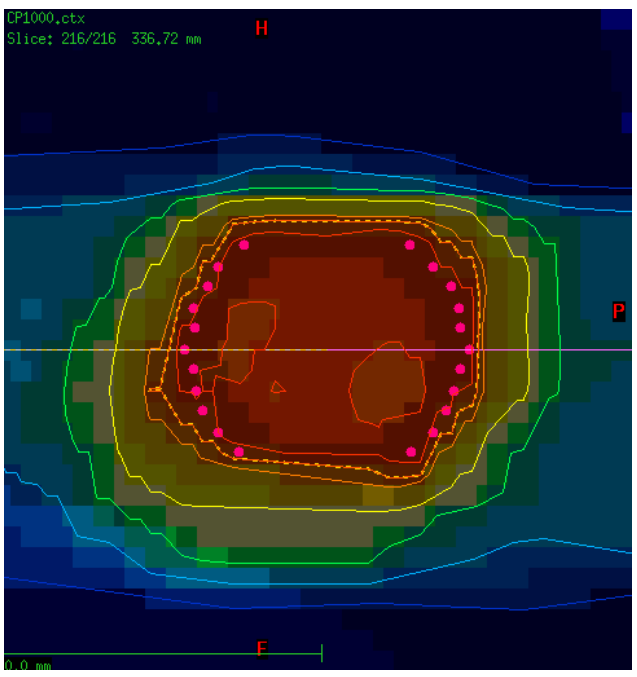

Figure 3.14: Dose distribution when ITV plan is applied to case17. Pink dots represent exhale CTV.

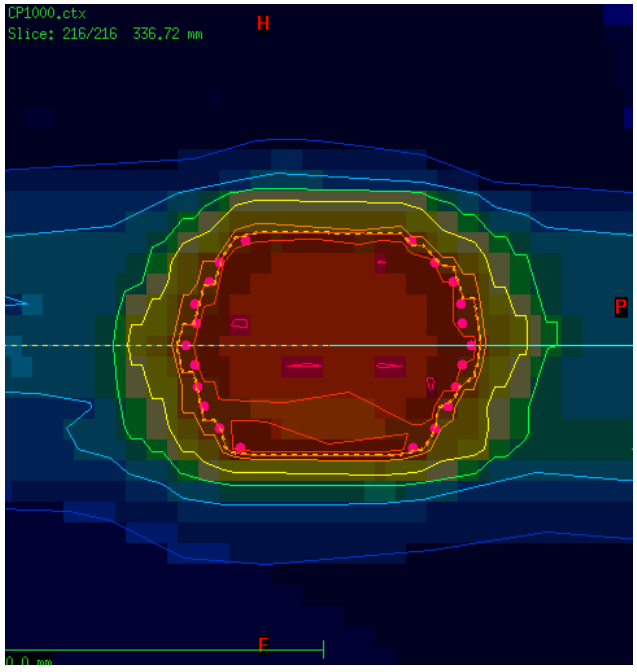

Figure 3.15: Dose distribution when average pdf plan is applied to case17. Pink dots represent exhale CTV.

For a comparative study beam intensity maps of the plans made using the different approaches are shown in Figure 3.16 and the corresponding statistics are shown in Table 3.5. The difference in the intensity maps is most visible in beam 4. As compared to the nominal plan, the average pdf intensity distribution is narrower, especially in particular bright pixels at the left side of the image of the nominal plan are not seen in the average pdf plan intensity map. Pixels at the center of the figure in the nominal 
Table 3.4: Dose statistics for ITV plan applied to motion variations scenario 1.(RL=right lung, $\mathrm{LL}=$ left lung, $\min / \mathrm{mean} / \max =\operatorname{minimum} /$ mean $/$ maximum dose in Gy)

\begin{tabular}{|c|c|c|c|c|c|}
\hline & ITV plan & case17 & case14 & case3 & case20 \\
\hline RL(max) & 61.0 & 62.2 & 62.3 & 62.2 & 62.2 \\
\hline LL(max) & 15.8 & 14.6 & 14.6 & 14.7 & 14.6 \\
\hline Cord(max) & 12.0 & 11.6 & 11.7 & 11.6 & 11.6 \\
\hline Body(max) & 45.6 & 42.1 & 42.0 & 42.1 & 42.0 \\
\hline Heart(max) & 32.8 & 28.9 & 28.7 & 28.9 & 28.6 \\
\hline CTV(min) & 58.4 & 57.9 & 57.3 & 57.6 & 57.6 \\
\hline CTV (mean) & 60.9 & 60.5 & 60.5 & 60.5 & 60.5 \\
\hline CTV(max) & 63.4 & 63.0 & 63.2 & 62.9 & 63.0 \\
\hline V20(\%-lung) & 26.9 & 26.3 & 26 & 26 & 25.7 \\
\hline V95(\%) & 100 & 100 & 100 & 100 & 100 \\
\hline
\end{tabular}

plan get brighter in the average pdf plan making the intensity distribution more uniform. This makes the plan less sensitive to motion variations. Similarly, the worst case pdf intensity distribution looks brighter and more uniform than the intensity maps of both the nominal and the average pdf plan, especially pixels at the center of the images. However, the intensity differences between these figures is very small, as can be seen from the statistics in Table 3.5 and therefore their sensitivity to motion variations are also not greatly different. But in the case of the ITV plan the beam intensity distribution covers a larger area and is more uniform compared to the nominal and robust plans. 


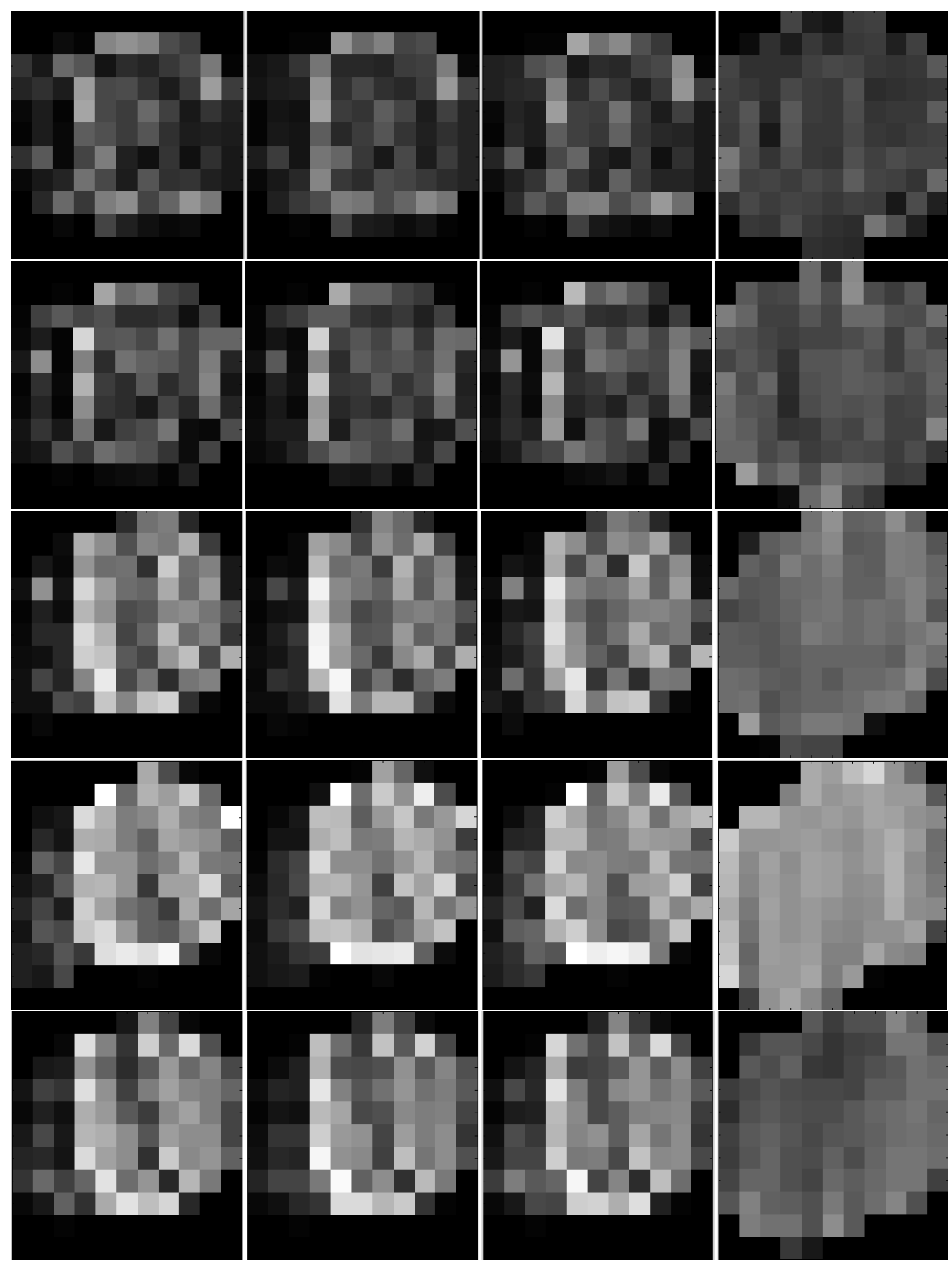

Figure 3.16: Left to right: beam intensity profiles of plans in nominal, average pdf, worst case pdf and ITV approach. Top to bottom: beam 1 to beam 5 . 


\begin{tabular}{|c|c|c|c|}
\hline $\begin{array}{c}\text { Nominal } \\
\text { plan }\end{array}$ & mean & std & $\begin{array}{c}\text { coefficient } \\
\text { of variation }\end{array}$ \\
\hline Beam 1 & 8.10 & 6.87 & 0.85 \\
\hline beam 2 & 8.25 & 7.35 & 0.89 \\
\hline Beam 3 & 13.38 & 10.73 & 0.80 \\
\hline Beam 4 & 16.11 & 12.08 & 0.75 \\
\hline Beam 5 & 14.37 & 10.73 & 0.74 \\
\hline
\end{tabular}

(a)

\begin{tabular}{|c|c|c|c|}
\hline $\begin{array}{c}\text { Worst } \\
\text { case pdf }\end{array}$ & mean & std & $\begin{array}{c}\text { coefficient } \\
\text { of variation }\end{array}$ \\
\hline Beam 1 & 8.06 & 6.59 & 0.82 \\
\hline Beam 2 & 8.36 & 7.60 & 0.90 \\
\hline Beam 3 & 13.36 & 10.61 & 0.79 \\
\hline Beam 4 & 16.18 & 11.81 & 0.73 \\
\hline Beam 5 & 14.24 & 10.44 & 0.73 \\
\hline
\end{tabular}

(c)

\begin{tabular}{|c|c|c|c|}
\hline $\begin{array}{c}\text { Average } \\
\text { pdf }\end{array}$ & mean & std & $\begin{array}{c}\text { coefficient } \\
\text { of variation }\end{array}$ \\
\hline Beam 1 & 8.00 & 6.54 & 0.82 \\
\hline Beam 2 & 8.28 & 7.24 & 0.87 \\
\hline Beam 3 & 13.01 & 10.81 & 0.83 \\
\hline Beam 4 & 15.94 & 12.43 & 0.78 \\
\hline Beam 5 & 13.89 & 10.82 & 0.77 \\
\hline
\end{tabular}

(b)

\begin{tabular}{|c|c|c|c|}
\hline $\begin{array}{c}\text { ITV } \\
\text { plan }\end{array}$ & mean & std & $\begin{array}{c}\text { coefficient } \\
\text { of variation }\end{array}$ \\
\hline Beam 1 & 9.80 & 2.98 & 0.30 \\
\hline Beam 2 & 13.18 & 3.58 & 0.27 \\
\hline Beam 3 & 16.01 & 3.39 & 0.21 \\
\hline Beam 4 & 23.13 & 4.57 & 0.19 \\
\hline Beam 5 & 14.27 & 3.24 & 0.23 \\
\hline
\end{tabular}

(d)

Table 3.5: Beam intensity statistics for (a) nominal (b) average pdf (c) worst case pdf and (d) ITV plan.

\subsection{Motion variation scenario 2: Amplitude variation}

In this scenario of motion variation the tumor is displaced with different amplitudes. The tested motion variations are shown in Figure 3.17. There are five cases. Case24 represents maximum displacement and case 23 represents minimum displacement. The other three cases lie between these two.

\subsubsection{Nominal 4D plan}

As said before, the nominal 4D plan does not consider the variation in motion. We chose case 22 as the pdf for the nominal plan as the tumor displacement in this case is smaller than two cases (case24 and case25) and bigger than two cases (case23 and case26). Figure 3.18 and Table 3.6 show the dose volume histograms and dose statistics of the nominal plan where it is applied to all the test cases. It is clear from this graph 


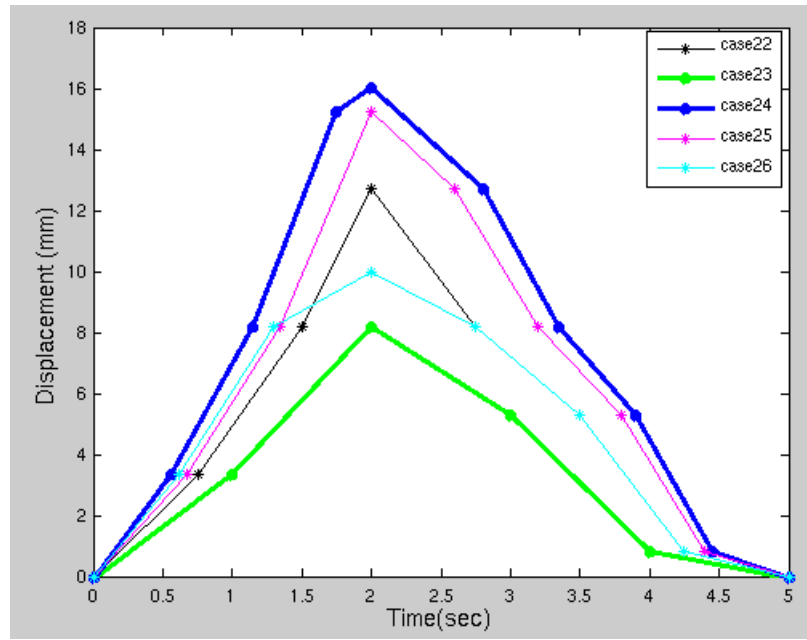

Figure 3.17: Tumor motion amplitude variation scenarios. The bold lines represent worst case pdfs.

that the nominal plan is highly sensitive to amplitude variations.

Table 3.6: Dose statistics for nominal 4D plan applied to amplitude variation scenario. $(\mathrm{RL}=$ right lung, $\mathrm{LL}=$ left lung, $\max / \mathrm{maen} / \mathrm{min}=\operatorname{maximum} / \mathrm{mean} / \mathrm{minimum}$ dose in $\mathrm{Gy})$

\begin{tabular}{|c|c|c|c|c|c|}
\hline & case22 & case23 & case24 & case25 & case26 \\
\hline RL(max) & 62.7 & 71.51 & 63.4 & 63.3 & 62.8 \\
\hline LL(max) & 14.1 & 14.7 & 13.7 & 39.9 & 14.0 \\
\hline Cord(max) & 17.3 & 17.6 & 17.0 & 17.2 & 17.2 \\
\hline Body(max) & 39.4 & 42.4 & 39.4 & 39.4 & 39.4 \\
\hline Heart(max) & 27.1 & 29.6 & 25.2 & 26.3 & 27.3 \\
\hline CTV (min) & 59.2 & 54.2 & 47.8 & 53.5 & 57.8 \\
\hline CTV (mean) & 61.9 & 62.6 & 60.3 & 61.2 & 61.9 \\
\hline CTV(max) & 64.4 & 74.9 & 68.5 & 66.4 & 65.0 \\
\hline V20(\%-lung) & 17.0 & 16.7 & 16 & 17 & 16.6 \\
\hline V95(\%) & 100 & 98.3 & 81.9 & 93.7 & 100 \\
\hline
\end{tabular}

\subsubsection{Robust plan using average pdf}

As before the average pdf is calculated from the cases in scenario 2 where the amplitude changes from $8.2 \mathrm{~mm}$ (case23) to $16.01 \mathrm{~mm}$ (case24). To test the sensitivity of the 
average pdf plan we applied this plan to all other cases. Figure 3.19 and Table 3.7 show the dose volume histogram and dose statistics of the average pdf plan and applied to all the test cases. The average pdf plan is not robust as can be seen in the graph and the table. The CTV is highly underdosed in case24 (Figure 3.20) for which the amplitude is largest. This makes sense because if we look at the weights of the average pdf (Table 2.3). In the average pdf the weight of phase 4, which is closest to inhale, is 0.034 . However, in case 24 the weight of that phase is 0.111 . So in the case 24 , the tumor is spending more time in phase 4 than is accounted for in the average pdf plan. As can be seen in Figure 3.20, the distal end of the tumor, which will move out of the beam as the tumor displacement increases, is underdosed.

Table 3.7: Dose statistics of average pdf plan applied to amplitude variation scenario. $(\mathrm{RL}=$ right lung, $\mathrm{LL}=$ left lung, $\max / \mathrm{maen} / \mathrm{min}=\operatorname{maximum} / \mathrm{mean} / \mathrm{minimum}$ dose in Gy)

\begin{tabular}{|c|c|c|c|c|c|c|}
\hline & $\begin{array}{c}\text { average } \\
\text { pdf plan }\end{array}$ & case22 & case23 & case24 & case25 & case26 \\
\hline RL(max) & 62.6 & 62.6 & 72.9 & 63.2 & 63 & 62.9 \\
\hline LL(max) & 14.3 & 14.3 & 14.8 & 14.2 & 14.2 & 14.2 \\
\hline Cord(max) & 18.3 & 18.4 & 18.7 & 18 & 18.2 & 18.3 \\
\hline Body(max) & 42.3 & 42.7 & 45.6 & 39.3 & 41 & 42.7 \\
\hline Heart(max) & 27.5 & 27.8 & 30.5 & 26.6 & 27 & 27.9 \\
\hline CTV (min) & 59.3 & 58.2 & 54.4 & 49.4 & 55.0 & 57.8 \\
\hline CTV(max) & 64.4 & 65.8 & 77.6 & 68.3 & 66.0 & 66.6 \\
\hline V20(\%-lung) & 17.4 & 17.4 & 19 & 16.9 & 17.5 & 17.1 \\
\hline V95(\%) & 100 & 100 & 98.3 & 84.3 & 96.2 & 100 \\
\hline
\end{tabular}

Furthermore, in case 23 the CTV max dose is more than the ICRU criteria. The accumulated dose distribution is shown in Figure 3.21. It can be seen that there is an area of high dose of the distal end of the tumor. Case 23 corresponds to when the tumor has the smallest displacement. If we compare the pdfs, in case 23 exhale phases are weighted more than in average pdf and inhale phases have no weight at all. This leads to over dosage to the CTV. This can be explained by examining the dose distributions obtained if we apply the nominal or average pdf plan to the exhale phase (Figure 3.22). 


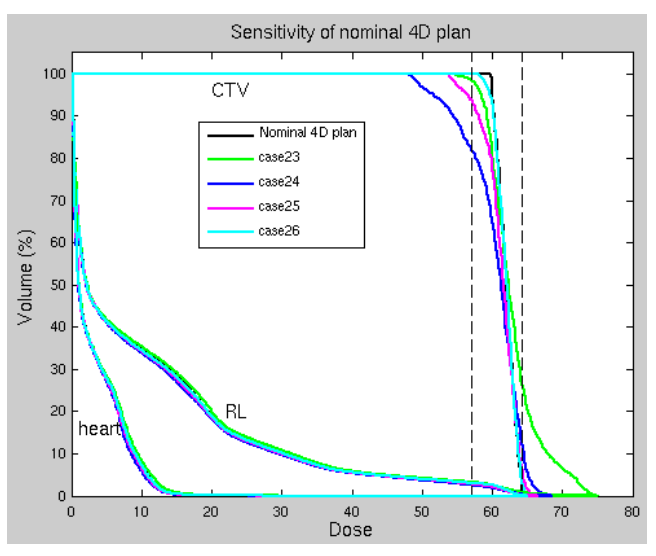

Figure 3.18: CTV DVH of nominal plan under amplitude variation.

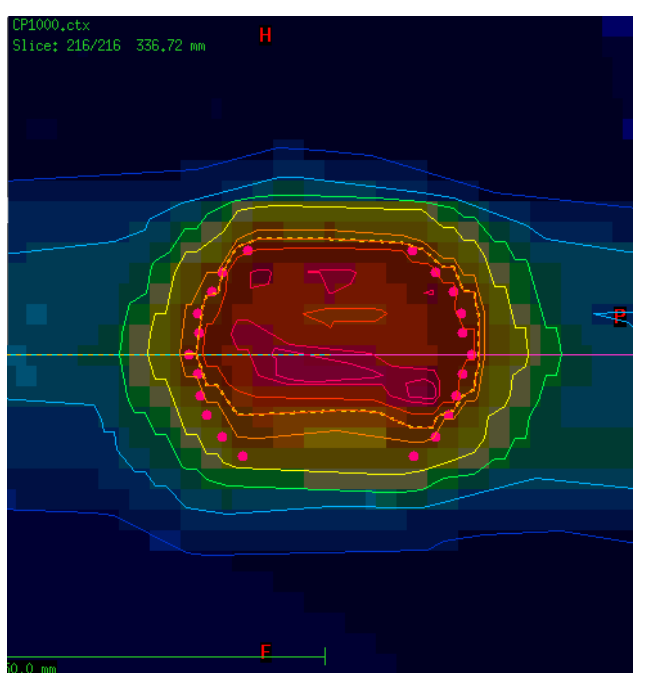

Figure 3.20: Sagital view of dose distribution when average pdf plan is applied to case24.

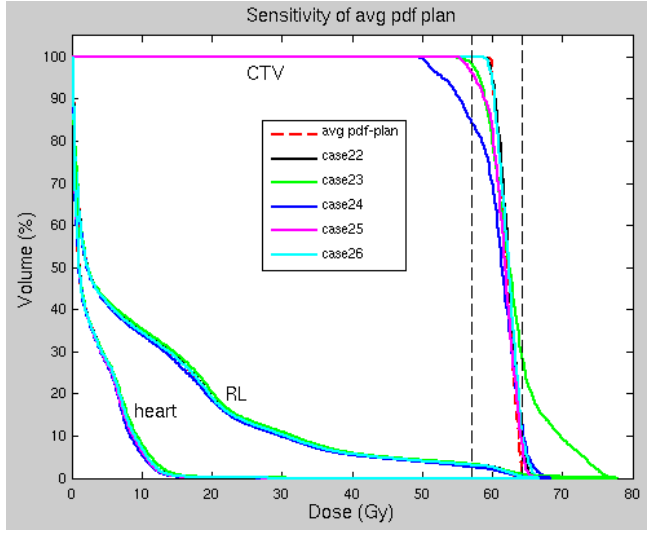

Figure 3.19: CTV DVH of average pdf plan under amplitude variation.

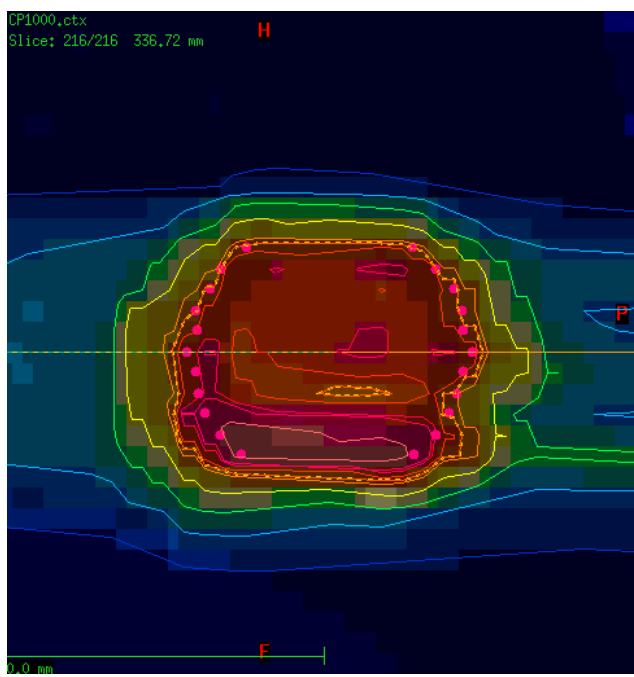

Figure 3.21: Sagital view of dose distribution when average pdf plan is applied to case23.

It can be seen that there is high dose at the distal end of the tumor. If the amplitude is decreased, the tumor spends more time in this region which explains the tumor over dosage. Despite the CTV dose not meeting the plan criteria for case 23, 24 and 25, the average pdf plan better accounts for the amplitude variation compared to the nominal 

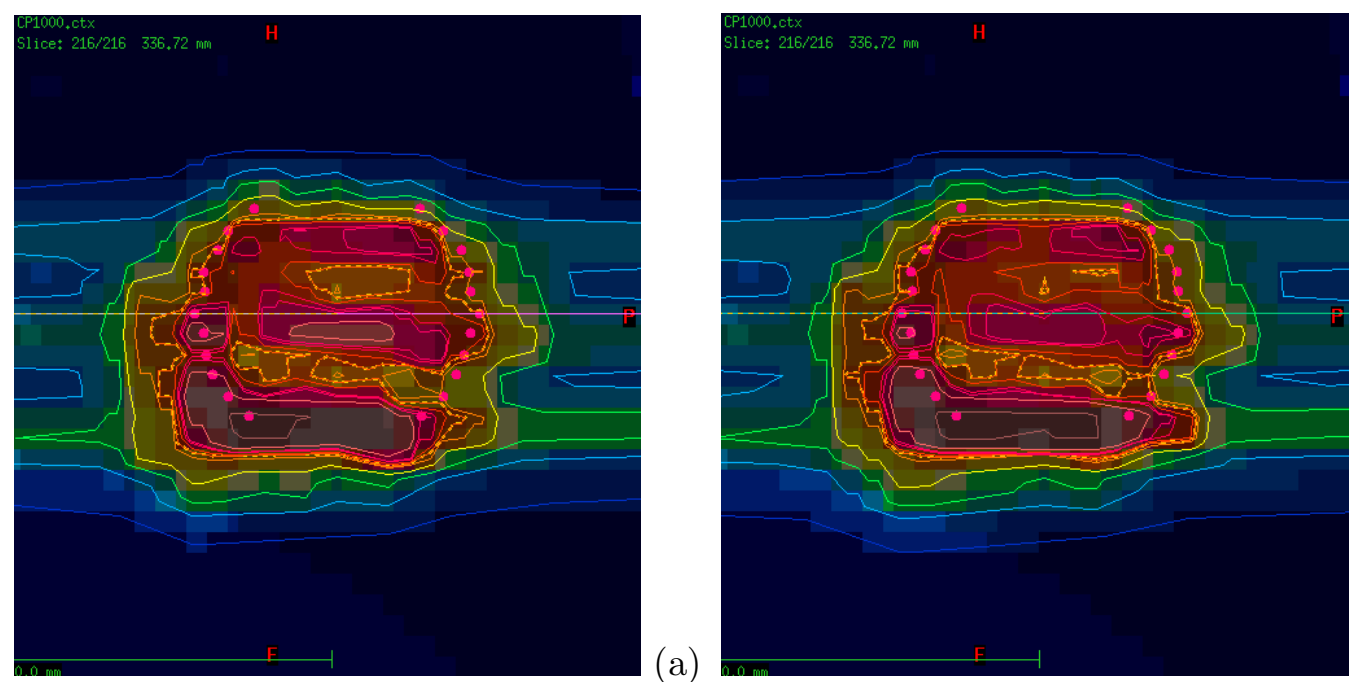

(b)

Figure 3.22: Dose distribution of (a) nominal and (b) average pdf plan applied to exhale CTV. Pink dots represent exhale CTV.

plan under same amplitude variations. If we compare with the phase weights of the average pdf to the phase weights of case22, which was used for the nominal plan, we see that the average pdf puts more weights on phase 4 and 6 , which means that dose is delivered to those phases which improves coverage in case 24 and case 25 .

\subsubsection{Robust plan using worst case pdf}

The two maximum amplitude variation cases, case23 and case24, were chosen as the worst case pdfs. Nominal $4 \mathrm{D}$ plans were made for these pdfs. Their beam weight files were combined and applied to each of the amplitude variation cases. At first they were combined with equal weights and were applied to each of the cases to test the sensitivity under amplitude variation. This resulted in robust plan sensitive to amplitude variation. As shown in Figure 3.23, except for case22, the CTV is underdosed. In another attempt to improve the robustness, the beam weight files were combined with different weights. Figures 3.24 - 3.26 show the resulting DVH with the weights applied to case 24 and 23 
are 70-30, 80-20 and 60-40.

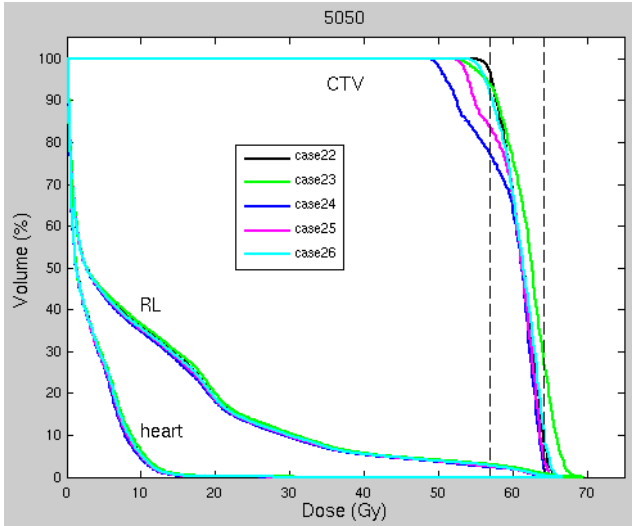

Figure 3.23: Dose volume histogram of wc pdf plan (50/50 ratio) under amplitude variation.

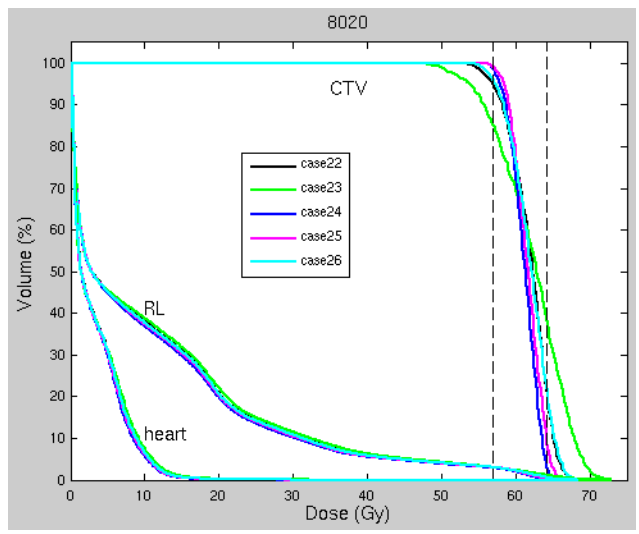

Figure 3.25: Dose volume histogram of wc pdf plan (80/20 ratio) under amplitude variation.

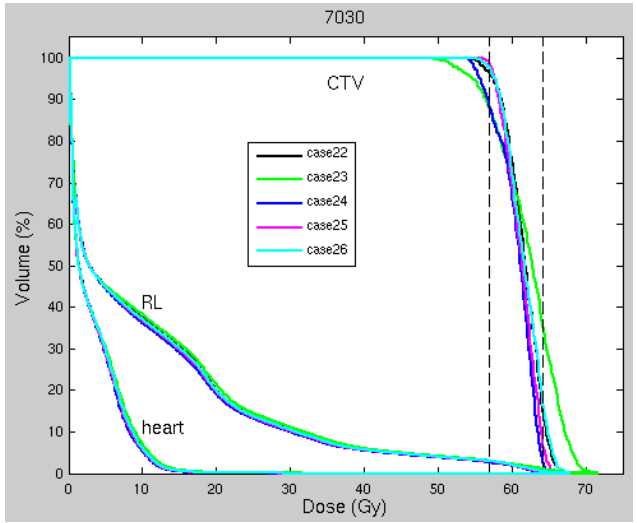

Figure 3.24: Dose volume histogram of wc pdf plan (70/30 ratio) under amplitude variation.

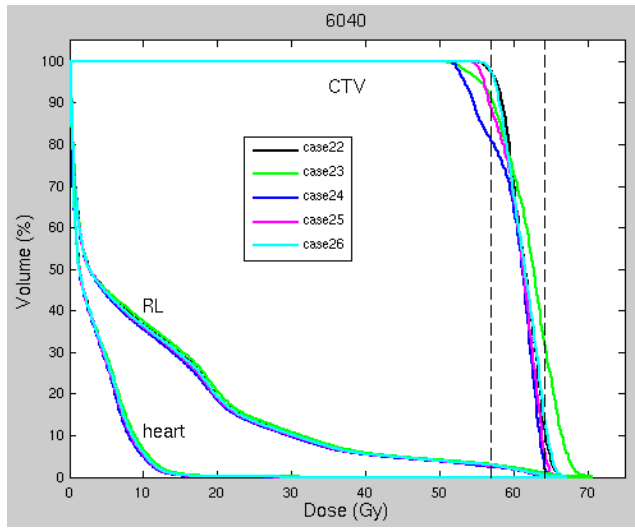

Figure 3.26: Dose volume histogram of wc pdf plan (60/40 ratio) under amplitude variation.

Looking at the DVH and the dose statistics (Table 3.8 and 3.9) none of these ratios produced a plan that was robust to all the tested variations. Compared to others, 70/30 and 80/20 ratios produce relatively better results. If we compare CTV(min) and V95 the combination $80 / 20$ produces almost robust plan except in case23, whereas combination $70 / 30$ does not change case 23 much, but worsens the case 24 and case 25 slightly and 
Table 3.8: Dose statistics for worst case pdf plan (combination ratio 70/30) applied to amplitude variation scenarios ( $\mathrm{RL}=$ right lung, $\mathrm{LL}=$ left lung, $\max / \mathrm{maen} / \mathrm{min}=\operatorname{maximum} / \mathrm{mean} / \operatorname{minimum}$ dose in Gy).

\begin{tabular}{|c|c|c|c|c|c|}
\hline & case22 & case23 & case24 & case25 & case26 \\
\hline RL(max) & 63.9 & 69.1 & 62.1 & 62.3 & 63.8 \\
\hline LL(max) & 15.2 & 16.1 & 14.4 & 14.7 & 15.1 \\
\hline Cord(max) & 16.6 & 16.6 & 16.6 & 16.6 & 16.6 \\
\hline Body(max) & 40.5 & 42.7 & 40.0 & 39.6 & 40.5 \\
\hline Heart(max) & 29.4 & 31.5 & 27.1 & 28.2 & 29 \\
\hline CTV (min) & 53.2 & 48.9 & 53.9 & 54.8 & 53.7 \\
\hline CTV(max) & 68 & 71.6 & 64.9 & 66.1 & 67.8 \\
\hline V20(\%-lung) & 18 & 19.4 & 17.1 & 17.7 & 18 \\
\hline V95(\%) & 96.2 & 87.8 & 88.3 & 98 & 97.3 \\
\hline
\end{tabular}

Table 3.9: Dose statistics for worst case pdf plan (combination ratio 80/20) applied to amplitude variation scenarios ( $\mathrm{RL}=$ right lung, $\mathrm{LL}=$ left lung, $\max / \mathrm{maen} / \mathrm{min}=\operatorname{maximum} / \mathrm{mean} /$ minimum dose in Gy).

\begin{tabular}{|c|c|c|c|c|c|}
\hline & case22 & case23 & case24 & case25 & case26 \\
\hline RL(max) & 64.4 & 70.2 & 61.9 & 62.5 & 65.4 \\
\hline LL(max) & 15.2 & 16.3 & 14.4 & 14.7 & 15.1 \\
\hline Cord(max) & 17.2 & 17.3 & 17.2 & 17.2 & 17.2 \\
\hline Body(max) & 40.9 & 43.1 & 40.5 & 40.0 & 40.4 \\
\hline Heart(max) & 30.0 & 32.0 & 27.8 & 28.9 & 29.6 \\
\hline CTV (min) & 52.1 & 47.4 & 55.9 & 55.5 & 52.8 \\
\hline CTV(max) & 68.7 & 72.7 & 65.1 & 66.5 & 68.4 \\
\hline V20(\%-lung) & 18.3 & 21.0 & 17.7 & 18.6 & 19.0 \\
\hline V95(\%) & 94.9 & 88.7 & 98.3 & 98.9 & 95.8 \\
\hline
\end{tabular}

improves the case 22 and case 26 slightly. The long tail of CTV DVH in case 23 implies that part of CTV volume is overdosed as is seen in the Figure 3.27. This approach of making robust plan is not working for amplitude variation and requires further research. The selection of the worst case pdfs should be investigated to see if it could improve this approach. 


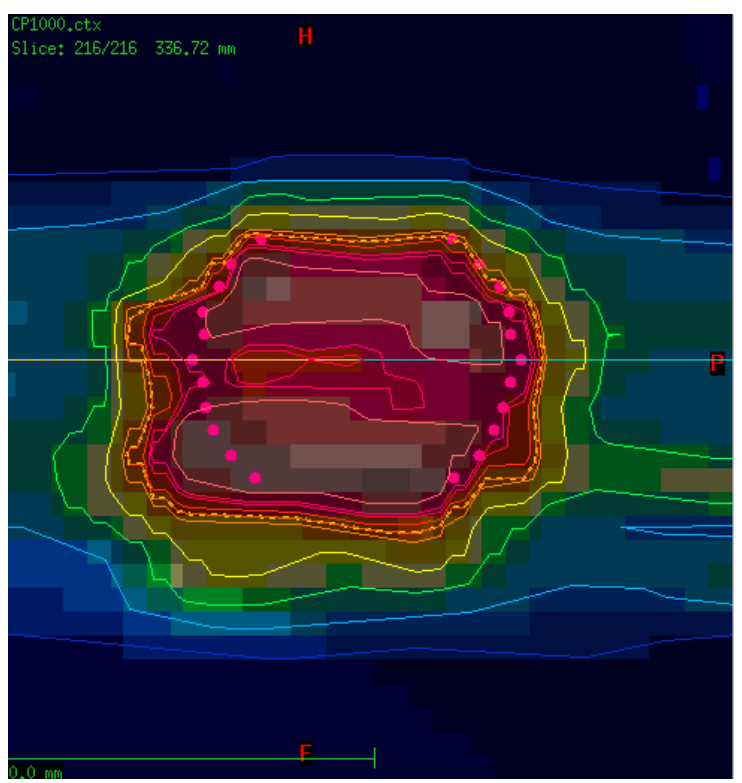

Figure 3.27: Dose distribution when worst case pdf plan (80-20 combination) is applied to case23. Yellow dashed line indicates $95 \%$ isodose. Pink dots represent the exhale CTV.

\subsubsection{ITV plan}

Once again, the average pdf plan and worst case pdf plans were compared with an ITV plan under the same amplitude variations. An ITV was designed which combined the CTV at phase 1 and phase 7 to be consistent with case22. As expected the ITV plan covered the CTV in each case. The DVHs are shown in Figure 3.28 and corresponding dose statistics in Table 3.10. As before, the ITV plan is robust to motion variation as the V95 is better compared to average pdf plan or worst case pdf plans in all cases. However, the V20 for lung is large for the ITV plan compared to the 4D plans. Also, the ITV plan puts extra dose outside the CTV (Figure 3.30). 


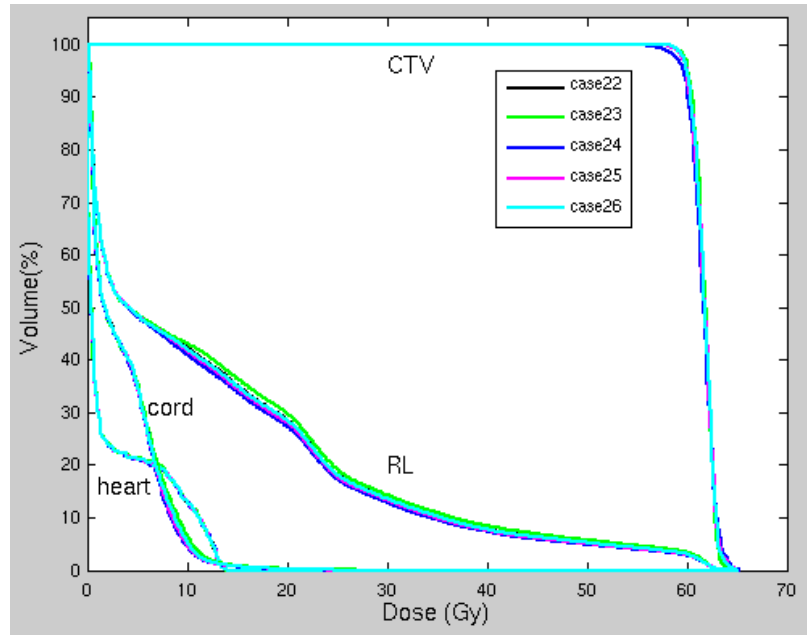

Figure 3.28: Dose volume histograms of the ITV plan under amplitude variation.

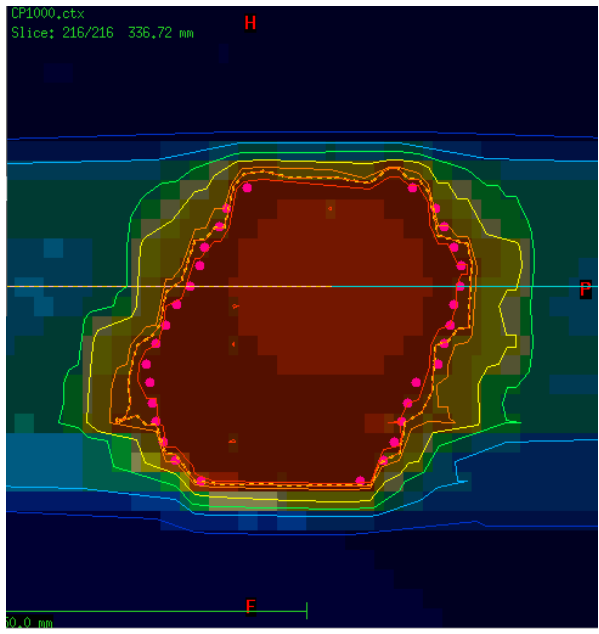

Figure 3.29: Dose distribution of the ITV plan on exhale phase.

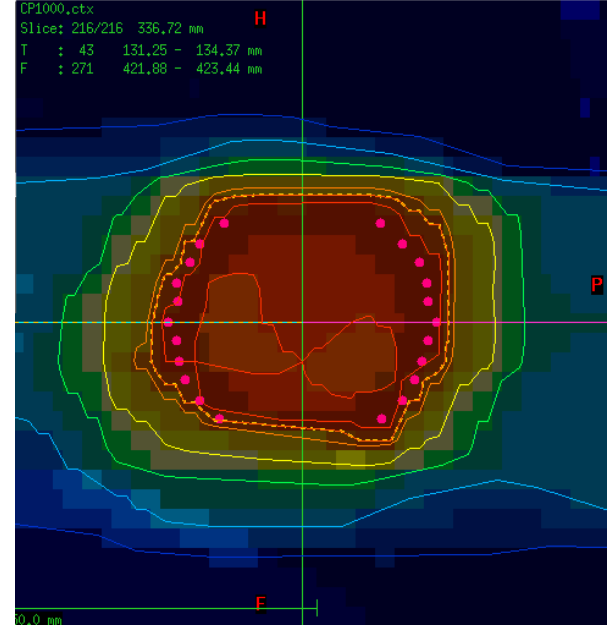

Figure 3.30: Accumulated dose distribution of the ITV plan applied to case 24 . 
Table 3.10: Dose statistics for ITV plan applied to amplitude variation scenario ( $\mathrm{RL}=$ right lung, $\mathrm{LL}=$ left lung, $\max / \mathrm{maen} / \mathrm{min}=\operatorname{maximum} / \mathrm{mean} /$ minimum dose in Gy).

\begin{tabular}{|c|c|c|c|c|c|c|}
\hline & $\begin{array}{l}\text { ITV } \\
\text { plan }\end{array}$ & case22 & case23 & case24 & case25 & case26 \\
\hline RL(max) & 62.0 & 63.3 & 63.5 & 63.5 & 63.5 & 63.4 \\
\hline LL(max) & 15.5 & 13.3 & 13.8 & 13.2 & 13.1 & 13.3 \\
\hline Cord(max) & 14.3 & 14.0 & 14.0 & 14.0 & 14.0 & 14.0 \\
\hline Body(max) & 41.0 & 32.8 & 32.6 & 33.6 & 33.2 & 32.9 \\
\hline Heart(max) & 26.67 & 24.6 & 25.9 & 23.6 & 24.0 & 24.5 \\
\hline CTV(min) & 59.5 & 56.7 & 57.3 & 54.0 & 56.3 & 56.7 \\
\hline CTV(max) & 64.3 & 64.9 & 64.5 & 65.2 & 65.1 & 65.0 \\
\hline V20(\%-lung) & 29.0 & 28.0 & 29.0 & 27.0 & 27.0 & 27.5 \\
\hline V95(\%) & 100 & 99.9 & 100 & 99.5 & 99.9 & 99.9 \\
\hline
\end{tabular}




\section{Chapter 4}

\section{Conclusions and future work}

\subsection{Summary and conclusions}

Tumor motion is a major challenge in lung cancer radiotherapy. The effect of the tumor motion is dose blurring and the consequence is higher dose to the surrounding healthy tissue and lower dose to the tumor. This may increase normal tissue complication and lower the tumor control probability. Several methods have been proposed to address tumor motion due to respiration. One approach is $4 \mathrm{D}$ optimization, however it is sensitive to tumor motion variations. Robust optimization methods have been developed to account for respiratory motion but all of them required modification to the treatment

planning system. In this thesis two simple approaches to make $4 \mathrm{D}$ robust optimization were proposed.

The first approach, which we call average pdf, involved optimizing a 4D plan using the average of the patient pdfs. In the second approach, which we call worst case pdf, two $4 \mathrm{D}$ plans were optimized using the worst case pdfs and the beam weight files of these plans were combined to make a robust plan. These methods were tested for two different types of tumor motion variation scenarios. In one scenario, the patient spends a different amount of time inhaling vs exhaling. In the other scenario the amplitude of 
tumor displacement was changed.

The preliminary results are encouraging. In the inhaling vs exhaling variation scenario both methods gave robust plans. In the amplitude variation scenario, both the methods work for small variations. The worst case pdf approach resulted in plans that were more robust than average pdf approach. These methods were compared to nominal $4 \mathrm{D}$ optimization and ITV plans to see if the robust plans were advantageous over them. We found that the nominal plans were very sensitive to motion variation compared to the robust plan and, although it gave the most robust plans, the ITV plan puts extra dose outside CTV compromising the healthy tissue sparing. Therefore, if our proposed approaches can be improved, they may prove to be advantageous over the ITV approach.

Some of the limitations of this study include the inability to generate displacement binned 4D CT image using XCAT which hindered our ability to vary amplitude in equal intervals. Also, we did not investigate how the number of phases affects our results. Furthermore, imperfect image registration may affect our comparison of accumulated dose for the different plans. The $4 \mathrm{D}$ plans take into account the image registration but the ITV plan does not. Therefore the accumulated dose of the ITV plan may indicate lower CTV coverage than the actual coverage. Finally, time limitations made us unable to test more tumor motion variation scenarios.

\subsection{Future work}

Future work continuing from this study should include testing more motion variation scenarios such as varying the time in inhale and exhale phases as well as baseline variations. The influence of the number of respiratory phases should be tested by generating a large number of phases in a respiration cycle so that the inter phase distance is small. Furthermore, displacement binned 4D CT images should be generated for testing the amplitude variation scenario. Further research should also include the use of pdfs from real patient data. Generating pdfs from real patient data and making robust plans on 
real patient 4DCTs will give more realistic scenarios of tumor motion variation.

Finally, further investigation is required to understand why the CTV in average pdf plan is slightly under dosed in case14 under motion variation scenario 1 but not in case17 although they mirror each other. Other areas of improvement include the understanding of worst case pdf approach working well in scenario 1 but not in scenario 2. Further improvement to the worst case pdf approach in motion variation scenario 2 should be investigated by selecting different pdfs to be the worst case pdfs. For example, in the amplitude variation scenario, pdfs representing the average and the largest amplitude could be chosen as the as the worst case pdf. 


\section{Bibliography}

[1] Canadian Cancer Societys Steering Committee on Cancer Statistics. Canadian cancer statistics, @online, January 2013.

[2] F.M. Khan and FM Khan. The physics of radiation therapy, volume 4. Lippincott Williams \& Wilkins Philadelphia, 2003.

[3] Varian Medical System Inc.com. Treatment techniques, @online, May 2013.

[4] EB Podgorsak and Kristofer Kainz. Radiation oncology physics: A handbook for teachers and students. Medical Physics, 33:1920, 2006.

[5] T. Bortfeld, R. Schmidt-Ulrich, W. De Neve, and D.E. Wazer, editors. Image-Guided IMRT. Springer, 2006.

[6] T. Bortfeld et al. Effects of intra-fraction motion on IMRT dose delivery: statistical analysis and simulation. Physics in Medicine and Biology, 47(13):2203, 2002.

[7] J. Hanley, M.M. Debois, D. Mah, G.S. Mageras, A. Raben, K. Rosenzweig, B. Mychalczak, L.H. Schwartz, P.J. Gloeggler, W. Lutz, et al. Deep inspiration breath-hold technique for lung tumors: the potential value of target immobilization and reduced lung density in dose escalation. International Journal of Radiation Oncology* Biology* Physics, 45(3):603-611, 1999.

[8] WP Segars, M Mahesh, TJ Beck, EC Frey, and BMW Tsui. Realistic CT simulation using the 4D XCAT phantom. Medical Physics, 35:3800, 2008. 
[9] S Nill. Development and application of a multi-modality inverse treatment planning system [Ph.D thesis], 2001.

[10] American Cancer Society. Lung cancer (non-small cell), @online, January 2013.

[11] C.F. Mountain et al. Revisions in the international system for staging lung cancer. chest, 111(6):1710-1717, 1997.

[12] Canadian Cancer Society. Treatment options at a glance,@online, April 2013.

[13] Cancer Research UK. Types of treatment for lung cancer, @online, April 2013.

[14] Kara Lynne Leonard, Thomas A DiPetrillo, John J Munro, and David E Wazer. A novel ytterbium-169 brachytherapy source and delivery system for use in conjunction with minimally invasive wedge resection of early-stage lung cancer. Brachytherapy, 10(2):163-169, 2011.

[15] Edward Yu, Craig Lewis, Ana Luisa Trejos, Rajni V Patel, and Richard A Malthaner. Lung cancer brachytherapy: Robotics-assisted minimally invasive approach. Current Respiratory Medicine Reviews, 7(5):340-353, 2011.

[16] International Commission on Radiation Units and Measurements. ICRU Report 50. Prescribing, recording, and reporting photon beam therapy. Bethesda, MD: ICRU, 1993.

[17] International Commission on Radiation Units and Measurements. ICRU Report 62. Prescribing, recording, and reporting photon beam therapy (supplement to ICRU Report 50). Bethesda, MD: ICRU, 1999.

[18] Frank H Attix, William C Roesch, and Eugene Tochilin. Introduction to Radiological Physics and Radiation Dosimetry, volume 1. Academic Press New York, 1968. 
[19] I.M.R.T.C. Working et al. Intensity-modulated radiotherapy: current status and issues of interest. International Journal of Radiation Oncology* Biology* Physics, 51(4):880-914, 2001.

[20] J.A. Purdy. Current ICRU definitions of volumes: limitations and future directions. volume 14, pages 27-40. Elsevier, 2004.

[21] Bodo Reitz and Moyed Miften. Comparison of the KonRad IMRT and Xio treatment planning systems. J Appl Clin Med Phys, 9(3):2770, 2008.

[22] Jake Van Dyk et al. The modern technology of radiation oncology, volume 2. Medical Physics Publ., 2005.

[23] A. Trofimov, E. Rietzel, H.M. Lu, B. Martin, S. Jiang, G.T.Y. Chen, and T. Bortfeld. Temporo-spatial IMRT optimization: concepts, implementation and initial results. Physics in Medicine and Biology, 50(12):2779, 2005.

[24] DM Shepard, MA Earl, XA Li, S Naqvi, and C Yu. Direct aperture optimization: a turnkey solution for step-and-shoot imrt. Medical Physics, 29:1007, 2002.

[25] Ergun E Ahunbay, Guang-Pei Chen, Steven Thatcher, Paul A Jursinic, Julia White, Katherine Albano, and X Allen Li. Direct aperture optimization-based intensitymodulated radiotherapy for whole breast irradiation. International Journal of $R a$ diation Oncology* Biology* Physics, 67(4):1248-1258, 2007.

[26] Paul J Keall, Gig S Mageras, James M Balter, Richard S Emery, Kenneth M Forster, Steve B Jiang, Jeffrey M Kapatoes, Daniel A Low, Martin J Murphy, Brad R Murray, et al. The management of respiratory motion in radiation oncology report of aapm task group 76. Medical Physics, 33:3874, 2006.

[27] Philippe Giraud, Yann De Rycke, Bernard Dubray, Sylvie Helfre, Daniel Voican, Ling Guo, Jean-Claude Rosenwald, Katia Keraudy, Martin Housset, Emmanuel 
Touboul, et al. Conformal radiotherapy (crt) planning for lung cancer: analysis of intrathoracic organ motion during extreme phases of breathing. International Journal of Radiation Oncology, Biology, Physics, 51(4):1081, 2001.

[28] Craig W Stevens, Reginald F Munden, Kenneth M Forster, Jason F Kelly, Zhongxing Liao, George Starkschall, Susan Tucker, and Ritsuko Komaki. Respiratorydriven lung tumor motion is independent of tumor size, tumor location, and pulmonary function. International Journal of Radiation Oncology* Biology* Physics, 51(1):62-68, 2001.

[29] Yvette Seppenwoolde, Hiroki Shirato, Kei Kitamura, Shinichi Shimizu, Marcel van Herk, Joos V Lebesque, and Kazuo Miyasaka. Precise and real-time measurement of $3 \mathrm{~d}$ tumor motion in lung due to breathing and heartbeat, measured during radiotherapy. International Journal of Radiation Oncology* Biology* Physics, 53(4):822-834, 2002.

[30] Hiroki Shirato, Yvette Seppenwoolde, Kei Kitamura, Rikiya Onimura, and Shinichi Shimizu. Intrafractional tumor motion: lung and liver. In Seminars in Radiation Oncology, volume 14, pages 10-18. Elsevier, 2004.

[31] T. Bortfeld, S.B. Jiang, and E. Rietzel. Effects of motion on the total dose distribution. In Seminars in Radiation Oncology, volume 14, pages 41-51. Elsevier, 2004 .

[32] C.S. Chui, E. Yorke, and L. Hong. The effects of intra-fraction organ motion on the delivery of intensity-modulated field with a multileaf collimator. Medical Physics, 30:1736, 2003.

[33] S.B. Jiang, C. Pope, K.M. Al Jarrah, J.H. Kung, T. Bortfeld, and G.T.Y. Chen. An experimental investigation on intra-fractional organ motion effects in lung imrt treatments. Physics in Medicine and Biology, 48(12):1773, 2003. 
[34] J Seco, GC Sharp, J Turcotte, D Gierga, T Bortfeld, and H Paganetti. Effects of organ motion on imrt treatments with segments of few monitor units. Medical Physics, 34:923, 2007.

[35] A.E. Lujan, E.W. Larsen, J.M. Balter, and R.K. Ten Haken. A method for incorporating organ motion due to breathing into 3D dose calculations. Medical Physics, 26:715, 1999.

[36] I Buzurovic, Y Yu, M Werner-Wasik, T Biswas, PR Anne, AP Dicker, and TK Podder. Implementation and experimental results of $4 \mathrm{~d}$ tumor tracking using robotic couch. Medical Physics, 39:6957, 2012.

[37] H. Shirato, S. Shimizu, K. Kitamura, T. Nishioka, K. Kagei, S. Hashimoto, H. Aoyama, T. Kunieda, N. Shinohara, H. Dosaka-Akita, et al. Four-dimensional treatment planning and fluoroscopic real-time tumor tracking radiotherapy for moving tumor. International Journal of Radiation Oncology* Biology* Physics, 48(2):435-442, 2000.

[38] JJ Nuyttens, J-B Prévost, J Praag, M Hoogeman, RJ Van Klaveren, PC Levendag, and PMT Pattynama. Lung tumor tracking during stereotactic radiotherapy treatment with the cyberknife: Marker placement and early results. Acta Oncologica, 45(7):961-965, 2006.

[39] Thomas Bortfeld, Timothy CY Chan, Alexei Trofimov, and John N Tsitsiklis. Robust management of motion uncertainty in intensity-modulated radiation therapy. Operations Research, 56(6):1461-1473, 2008.

[40] T.C.Y. Chan, T. Bortfeld, and J.N. Tsitsiklis. A robust approach to IMRT optimization. Physics in Medicine and Biology, 51(10):2567, 2006.

[41] Omid Nohadani, Joao Seco, and Thomas Bortfeld. Motion management with phaseadapted 4d-optimization. Physics in Medicine and Biology, 55(17):5189, 2010. 
[42] Fan Zhang, Jing Hu, Chris R Kelsey, David Yoo, Fang-Fang Yin, and Jing Cai. Reproducibility of tumor motion probability distribution function in stereotactic body radiation therapy of lung cancer. International Journal of Radiation Oncology* Biology* Physics, 2012.

[43] Emily Heath, Jan Unkelbach, and Uwe Oelfke. Incorporating uncertainties in respiratory motion into 4D treatment plan optimization. Medical Physics, 36:3059, 2009.

[44] Christian Vrančić, Alexei Trofimov, Timothy CY Chan, Gregory C Sharp, and Thomas Bortfeld. Experimental evaluation of a robust optimization method for imrt of moving targets. Physics in medicine and biology, 54(9):2901, 2009.

[45] German Cancer Research Center. Virtuos: Virtual Radiotherapy Simulator: User Mannual, @online, May 2013.

[46] Emily Heath, DL Collins, PJ Keall, L Dong, and J Seuntjens. Quantification of accuracy of the automated nonlinear image matching and anatomical labeling (ANIMAL) nonlinear registration algorithm for 4D CT images of lung. Medical Physics, 34:4409, 2007.

[47] D. Louis Collins and Alan C. Evans. ANIMAL: validation and applications of nonlinear registration-based segmentation. International Journal of Pattern Recognition and Artificial Intelligence, 11(08):1271-1294, 1997.

[48] J D Bradley, Kyounghwa Bae, Mary V Graham, Roger Byhardt, Ramaswamy Govindan, Jack Fowler, James A Purdy, Jeff M Michalski, Elizabeth Gore, and Hak Choy. Primary analysis of the phase II component of a phase I/II dose intensification study using three-dimensional conformal radiation therapy and concurrent chemotherapy for patients with inoperable non-small-cell lung cancer: RTOG 0117. Journal of Clinical Oncology, 28(14):2475-2480, 2010. 